\title{
Height pairings on Shimura curves and $p$-adic uniformization
}

by

\author{
Stephen S. Kudla ${ }^{1}$ \\ and \\ Michael Rapoport
}

\section{Introduction.}

In a recent paper $[\mathbf{1 5}]$ of one of us it was shown that there is a close connection between the value of the height pairing of certain arithmetic 0-cycles on Shimura curves and the values at the center of their symmetry of the derivatives of certain metaplectic Eisenstein series of genus 2. On the one hand, the height pairing can be written as a sum of local height pairings. For example, if the 0-cycles have disjoint support on the generic fiber, then their height pairing is a sum of an archimedean contribution and a contribution from each of the (finitely many) finite primes $p$ for which the cycles meet in the fiber at $p$. On the other hand, it turns out that the non-singular part of the Fourier expansion of the central derivative of the metaplectic Eisenstein series also has a decomposition into a sum of contributions indexed by the places of $\mathbb{Q}$. Then, one would like to compare the height pairing and the Fourier coefficients by proving an identity of local contributions place by place. In loc. cit. the identity for the archimedean place was proved, and it was shown that the identity at a non-archimedean place of good reduction is a consequence of results of Gross and Keating, [9], (for the algebraic-geometric side) and of Kitaoka, [11], (for the analytic side). It then remains to consider the finite primes $p$ where the Shimura curve has bad reduction. These are of two sorts: (i) the primes $p$ at which the quaternion algebra defining the Shimura curve is split, but which divide the level, and (ii) the primes $p$ at which the quaternion algebra remains division.

In the present paper we consider the case of a non-archimedean place $p$ of bad reduction of the second type, and hence where $p$-adic uniformization in the sense of Cherednik-Drinfeld holds. It turns out that the identity to be proved in this case can be reduced to a purely local statement concerning the Drinfeld $p$-adic upper half plane $\hat{\Omega}$ (the formal scheme version). Therefore the bulk of this paper (sections 1-7) is concerned with the local situation, and in this introduction we will concentrate on the local aspects of the problem.

\footnotetext{
${ }^{1}$ Partially supported by NSF Grant DMS-9622987
} 
Let $W=W\left(\overline{\mathbb{F}}_{p}\right)$ be the ring of Witt vectors of $\overline{\mathbb{F}}_{p}$. Also let $B$ be the division quaternion algebra over $\mathbb{Q}_{p}$ and let $O_{B}$ be its maximal order.

Recall that $\hat{\Omega} \times{ }_{\operatorname{Spf}} \mathbb{Z}_{p} \operatorname{Spf} W$ parametrizes pairs $(X, \varrho)$, where $X$ is a special formal (s.f.) $O_{B}$-module (of dimension 1 and height 2) over a $W$-scheme $S$ on which $p$ is locally nilpotent and

$$
\varrho: \mathbb{X} \times_{\text {Spec } \overline{\mathbb{F}}_{p}} \bar{S} \longrightarrow X \times_{S} \bar{S}
$$

is an $O_{B}$-linear quasi-isogeny of height 0 . Here $\mathbb{X}$ is a fixed s.f. $O_{B}$-module over Spec $\overline{\mathbb{F}}_{p}$, and $\bar{S}=S \times_{\text {Spec }} \mathbb{F}_{p}$ Spec $\overline{\mathbb{F}}_{p}$ is the special fibre of $S$. We note that $\operatorname{End}_{O_{B}}^{0}(\mathbb{X}) \simeq M_{2}\left(\mathbb{Q}_{p}\right)$.

We define cycles in $\hat{\Omega} \times_{\text {Spf } \mathbb{Z}_{p}}$ Spf $W$ by imposing additional endomorphisms as follows. Let

$$
V=\left\{j \in \operatorname{End}_{O_{B}}^{0}(\mathbb{X}) ; \operatorname{tr}^{0}(j)=0\right\}
$$

equipped with the quadratic form $q=-$ det, also given by $j^{2}=q(j) \cdot \mathrm{id}$. We call the elements of $V$ special endomorphisms. For any $j \in V$ with $q(j) \in \mathbb{Z}_{p} \backslash\{0\}$ we define a special cycle $Z(j)$ which is a closed formal subscheme of $\hat{\Omega} \times \operatorname{Spf} \mathbb{Z}_{p}$ Spf $W$. It is the locus of pairs $(X, \varrho)$ such that $\varrho \circ j \circ \varrho^{-1}$ extends to an isogeny of $X$.

We assume, from now on, that $p \neq 2$.

Our first task is to investigate the structure of a single special cycle. For this we use two methods. The first is completely elementary and uses the Bruhat-Tits building of $P G L_{2}\left(\mathbb{Q}_{p}\right)$. This method is sufficient to give a fairly accurate picture of the point set of the special fibre of $Z(j)$. More precisely, let $\mathcal{B}$ be the Bruhat-Tits building of $P G L_{2}\left(\mathbb{Q}_{p}\right)$, and recall that the irreducible components of the special fiber of $\hat{\Omega} \times{ }_{\operatorname{Spf} \mathbb{Z}_{p}}$ Spf $W$ are projective lines indexed by the vertices of $\mathcal{B}$, i.e., by homothety classes $[\Lambda]$ of $\mathbb{Z}_{p}$-lattices $\Lambda$ in $\mathbb{Q}_{p}^{2}$. If $\mathbb{P}_{[\Lambda]}$ is the component corresponding to $[\Lambda]$, then $Z(j) \cap \mathbb{P}_{[\Lambda]} \neq \emptyset$ if and only if $j(\Lambda) \subset \Lambda$. It follows that the support of $Z(j)$ is contained in the union of the $\mathbb{P}_{[\Lambda]}$ 's for $[\Lambda]$ 's lying in the tube $\mathcal{T}(j)$ of radius $\frac{1}{2}$ ord $q(j)$ around the fixed point set $\mathcal{B}^{j}$ of $j$ in $\mathcal{B}$. The second method is due to Genestier, [7]. His crucial observation is that $Z(j)$ may be identified with the fixed point locus on $\hat{\Omega} \times{ }_{\operatorname{Spf} \mathbb{Z}_{p}} \operatorname{Spf} W$ of the action of $j$ if $\operatorname{ord}_{p}(q(j))=0$ (resp. of id $+j$ if $\operatorname{ord}_{p}(q(j))>0$ ). This observation allows one to write explicit local equations for the cycle $Z(j)$ and thus yields a good understanding of its local structure. The combination of the two methods 
determines $Z(j)$ completely and shows that, apart from degenerate cases, $Z(j)$ is purely one-dimensional. Moreover, $Z(j)$ can contain (multiples of) irreducible components of the special fibre and can even have embedded components! These latter phenomena are in contrast to the case of good reduction. At the end of the introduction there is a schematic picture of the various possibilities of $Z(j)$.

We next turn to the calculation of the intersection product $\left(Z(j), Z\left(j^{\prime}\right)\right)$ of two special cycles, assuming that $j$ and $j^{\prime}$ span a 2-dimensional non-degenerate quadratic $\mathbb{Z}_{p}$-submodule $\mathbf{j}$ of $V$. In contrast with the case of good reduction, we have to deal here with cases of excess intersection. We proceed in two steps. We first prove that $\left(Z(j), Z\left(j^{\prime}\right)\right)$ only depends on the $\mathbb{Z}_{p}$-span $\mathbf{j}$ of $j$ and $j^{\prime}$. This is achieved by showing that the Genestier equations globalize to give a resolution of the structure sheaves of $Z(j)$ and $Z\left(j^{\prime}\right)$. This part of our paper is in the spirit of the venerable theory of Möbius transformations. In the case of good reduction the analogue of this independence statement is trivial, whereas at an archimedean place it was one of the main and most difficult steps in the proof of the local identity, [15]. Since $p \neq 2$, we may then assume that $j, j^{\prime}$ diagonalize the quadratic form on $\mathbf{j}$, i.e. that $j j^{\prime}=-j^{\prime} j$. In this case, the calculation of the intersection number becomes a piece of recreational mathematics, involving the various facts about the structure of special cycles mentioned above and combinatorial arguments involving the tubes $\mathcal{T}(j)$ in the building $\mathcal{B}$. The end result then is:

Theorem A. Let $j$ and $j^{\prime}$ be special endomorphisms with $q(j), q\left(j^{\prime}\right) \in \mathbb{Z}_{p} \backslash\{0\}$ such that their $\mathbb{Z}_{p}$-span $\mathbf{j}=\mathbb{Z}_{p} j+\mathbb{Z}_{p} j^{\prime}$ is of rank 2 and is nondegenerate for the quadratic form. Let

$$
T=\left(\begin{array}{cc}
q(j) & \frac{1}{2}\left(j, j^{\prime}\right) \\
\frac{1}{2}\left(j^{\prime}, j\right) & q\left(j^{\prime}\right)
\end{array}\right),
$$

and suppose that $T$ is $G L_{2}\left(\mathbb{Z}_{p}\right)$-equivalent to diag $\left(\varepsilon_{1} p^{\alpha}, \varepsilon_{2} p^{\beta}\right)$, where $\varepsilon_{1}, \varepsilon_{2} \in$ $\mathbb{Z}_{p}^{\times}$, and $\alpha$ and $\beta$ are integers with $0 \leq \alpha \leq \beta$. Then $\left(Z(j), Z\left(j^{\prime}\right)\right)=e_{p}(T)$ depends only on the $G L_{2}\left(\mathbb{Z}_{p}\right)$-equivalence class of $T$, and is given explicitly by:

$e_{p}(T)=\alpha+\beta+1- \begin{cases}p^{\alpha / 2}+2 \frac{p^{\alpha / 2}-1}{p-1} & \text { if } \alpha \text { is even and } \chi\left(\varepsilon_{1}\right)=-1, \\ (\beta-\alpha+1) p^{\alpha / 2}+2 \frac{p^{\alpha / 2}-1}{p-1} & \text { if } \alpha \text { is even and } \chi\left(\varepsilon_{1}\right)=1, \\ 2 \frac{p^{(\alpha+1) / 2}-1}{p-1} & \text { if } \alpha \text { is odd. }\end{cases}$

The entity $e_{p}(T)$ appearing in this theorem may be related to local representation densities of quadratic forms and their derivatives. For simplicity, we continue to 
assume that $p \neq 2$. Recall that for nonsingular symmetric matrices $S \in \operatorname{Sym}_{m}\left(\mathbb{Z}_{p}\right)$ and $T \in \operatorname{Sym}_{n}\left(\mathbb{Z}_{p}\right)$, the classical representation density is defined by

$$
\alpha_{p}(S, T)=\lim _{t \rightarrow \infty} p^{-t n(2 m-n-1) / 2}\left|\left\{x \in M_{m, n}\left(\mathbb{Z} / p^{t} \mathbb{Z}\right) ; S[x]-T \in p^{t} \operatorname{Sym}_{m}\left(\mathbb{Z}_{p}\right)\right\}\right| .
$$

Let

$$
S=-\left(\begin{array}{lll}
1 & & \\
& 1 & \\
& & -1
\end{array}\right)
$$

be the matrix for the determinant quadratic form on the space $V\left(\mathbb{Z}_{p}\right)$ of special endomorphisms, i.e. on the lattice $\left\{x \in M_{2}\left(\mathbb{Z}_{p}\right) ; \operatorname{tr}(x)=0\right\}$, and let

$$
S^{\prime}=-\left(\begin{array}{lll}
\eta & & \\
& p & \\
& & -\eta p
\end{array}\right),
$$

be the matrix for the reduced norm quadratic form on the space

$$
V^{\prime}\left(\mathbb{Z}_{p}\right)=\left\{x \in O_{B} ; \operatorname{tr}(x)=0\right\}
$$

Here $\eta \in \mathbb{Z}_{p}^{\times}$with $\chi(\eta):=(\eta, p)_{p}=-1$, and $(a, b)_{p}$ is the quadratic Hilbert symbol for $\mathbb{Q}_{p}$. Also let

$$
S^{\prime \prime}=-\left(\begin{array}{lll}
1 & & \\
& p & \\
& & -p
\end{array}\right) \text {. }
$$

Then for a pair of special endomorphisms $j$ and $j^{\prime}$ with associated matrix $T$, as in Theorem A above, we have $\alpha_{p}\left(S^{\prime}, T\right)=0$. In this situation, it is possible, as in [15], to define the derivative $\alpha_{p}^{\prime}\left(S^{\prime}, T\right)$ of the representation density (see (7.4)). For the unimodular quadratic form $S$ and for any binary form $T$, the representation densities and their derivatives can be calculated using the results of Kitaoka, [11], [15], and Proposition 7.1 below. For $S^{\prime}$ and $S^{\prime \prime}$, the analogous information is provided by the work of B. Myers, [18], and T. Yang, [20].

These representation densities are then related to the intersection number $e_{p}(T)$ as follows: 
Theorem B. For a pair of special endomorphisms $j$ and $j^{\prime}$ with associated matrix $T$, as in Theorem A,

$$
e_{p}(T)=-\frac{1}{p+1} \alpha_{p}^{\prime}\left(S^{\prime},-T\right)+\frac{2 p^{2}}{p+1} \alpha_{p}(S,-T)+\frac{1}{2(p-1)} \alpha_{p}\left(S^{\prime \prime},-T\right)
$$

This result illustrates again the rather remarkable connection between arithmetic intersection numbers on certain moduli spaces on the one hand, and the arithmetic theory of quadratic forms on the other. For additional examples, see (at least) [9], [15], [16], and [17].

It is instructive to compare the statements of Theorems A and B with the following reformulation of the result of Gross and Keating, [9]. Changing notation slightly, we fix a formal p-divisible group $\mathbb{X}$ of dimension 1 and height 2 over Spec $\overline{\mathbb{F}}_{p}$, and let $\mathcal{M}$ be the moduli scheme where $\mathcal{M}(S)$ is the set of pairs $(X, \rho)$ for $\rho$ a quasi-isogeny of height 0 as in (0.1). Then, there is a (non-canonical) analogue of the Drinfeld isomorphism:

$$
\mathcal{M} \simeq \operatorname{Spf} W[[t]]
$$

Fix an isomorphism $\operatorname{End}^{0}(\mathbb{X})=B$, and let

$$
V^{\prime}=\left\{j \in B ; \operatorname{tr}^{0}(j)=0\right\}
$$

with (anisotropic) quadratic form defined by $j^{2}=q(j)$ id. To a special endomorphism $j \in V^{\prime}$ with $q(j) \neq 0$, define a cycle $Z(j) \subset \mathcal{M}$, as before.

Theorem. (Gross-Keating) (i) If $q(j) \notin \mathbb{Z}_{p}$, then $Z(j)=\emptyset$. Otherwise, $Z(j)$ is a divisor on $\mathcal{M}$, and is flat over $\operatorname{Spf} W$.

(ii) Let $j$ and $j^{\prime} \in V^{\prime}$ be special endomorphisms such that $j$ and $j^{\prime}$ span a 2dimensional non-degenerate subspace of $V^{\prime}$. Let $T=q\left(j, j^{\prime}\right)$ be the matrix of the quadratic form with respect to the basis $j, j^{\prime}$, as in Theorem A. Then $Z(j) \cap Z\left(j^{\prime}\right)$ has support at the origin in $\mathcal{M}$ and the intersection multiplicity is

$$
\left(Z(j), Z\left(j^{\prime}\right)\right)= \begin{cases}\sum_{i=0}^{(\alpha-2) / 2}(\alpha+\beta-4 i) p^{i}+\frac{1}{2}(\beta-\alpha+1) p^{\alpha / 2} & \text { if } \alpha \text { is even } \\ \sum_{i=0}^{(\alpha-1) / 2}(\alpha+\beta-4 i) p^{i} & \text { if } \alpha \text { is odd }\end{cases}
$$

In particular, this multiplicity depends only on the $G L_{2}\left(\mathbb{Z}_{p}\right)$-equivalence class of $T$. 
By Kitaoka's formula, Corollary 8.5 of [15], we then have the (simpler) analogue of Theorem B in this case:

$$
\left(Z(j), Z\left(j^{\prime}\right)\right)=-\frac{p^{2}}{p^{2}-1} \alpha_{p}^{\prime}(S, T) .
$$

Two features of the theory developed here are worth pointing out. Recall that the proofs of Gross and Keating in the case of good reduction make heavy use of the theory of (formal) complex multiplication which connects this case, via Gross's theory of quasi-canonical liftings, [8], with Kronecker's Jugendtraum. The first remark is that in our case this connection does not appear (explicitly! - it is of course hidden to some degree in Drinfeld's representability theorem). The second remark is that it is the global nature of the Cherednik-Drinfeld uniformization which allowed us here to prove the independence statement on the intersection numbers. In other, higher-dimensional cases $[\mathbf{1 6}],[\mathbf{1 7}]$ when the analogous independence property is problematical, global uniformization of the special fiber is not available. In these cases these problems remain a challenge.

In the last two sections, we draw the global consequences of the local results of sections 1-7 just described and obtain an extension of the results of [15].

For an indefinite quaternion algebra $B$ over $\mathbb{Q}$, let $H=B^{\times}$, and let $V=\{x \in$ $\left.B ; \operatorname{tr}^{0}(x)=0\right\}$. Fix a prime $p(p \neq 2)$ which ramifies in $B$ and let $K=K_{p} K^{p}$ be a compact open subgroup of $H\left(\mathbb{A}_{f}\right)$ such that $K_{p}=O_{B_{p}}^{\times}$, where $O_{B_{p}}$ denotes the maximal order in $B_{p}$. Associated to this data is a model $\mathcal{A}_{K}$ over $\mathbb{Z}_{(p)}$ of the Shimura curve $A_{K}$ over $\mathbb{Q}$ attached to $B$ and $K$. It is the moduli space of certain abelian surfaces with $O_{B}$-action and $K^{p}$-level structure, For each pair $(t, \omega)$ with $t \in \mathbb{Z}_{(p)}, t<0$ and $\omega \subset V\left(\mathbb{A}_{f}^{p}\right)$ a $K^{p}$-invariant compact open subset, there is a special cycle

$$
\mathcal{C}(t, \omega) \longrightarrow \mathcal{A}_{K}
$$

defined, as in [15], by imposing an additional special endomorphism. The generic fiber of $\mathcal{C}(t, \omega)$ is a $\mathbb{Q}$-rational 0 -cycle on the Shimura curve $A_{K}$. Given a pair of special cycles $\mathcal{C}_{1}=\mathcal{C}\left(t_{1}, \omega_{1}\right)$ and $\mathcal{C}_{1}=\mathcal{C}\left(t_{2}, \omega_{2}\right)$, we form their intersection

$$
\mathcal{C}=\mathcal{C}_{1} \times \mathcal{A}_{K} \mathcal{C}_{2}
$$

This scheme has a decomposition

$$
\mathcal{C}=\coprod_{T} \mathcal{C}_{T}
$$


where each $\mathcal{C}_{T}$ is a union of connected components of $\mathcal{C}$, and where $T \in \operatorname{Sym}_{2}\left(\mathbb{Z}_{(p)}\right)$ runs over negative semi-definite matrices with diagonal entries $t_{1}$ and $t_{2}$. For $\operatorname{det}(T) \neq 0$, the image of $\mathcal{C}_{T}$ lies in the special fiber of $\mathcal{A}_{K}$. Utilizing the p-adic uniformization of $\mathcal{A}_{K}$, and the results on intersections of special cycles in Drinfeld space, we obtain the following statement.

Theorem C. Assume that $t_{1} t_{2}$ is not a square in $\mathbb{Q}^{\times}$. Then the special cycles $\mathcal{C}\left(t_{1}, \omega_{1}\right)$ and $\mathcal{C}\left(t_{2}, \omega_{2}\right)$ do not meet in the generic fiber and their intersection number is given by

$$
\left(\mathcal{C}\left(t_{1}, \omega_{1}\right), \mathcal{C}\left(t_{2}, \omega_{2}\right)\right)=2 \sum_{T} e_{p}(T) \operatorname{vol}\left(K^{p}\right)^{-1} I_{T}\left(\varphi_{1}^{p} \otimes \varphi_{2}^{p}\right)
$$

where $e_{p}(T)$ is as in Theorem $A$ and $I_{T}\left(\varphi_{1}^{p} \otimes \varphi_{2}^{p}\right)$ is a certain orbital integral associated to the data $\omega_{1}, \omega_{2}$, and $T$. The summation runs over the same range as in (0.10).

Finally, this result, combined with Theorem B above yields the connection between the $p$ part of the height pairing of the cycles $\mathcal{C}_{1}$ and $\mathcal{C}_{2}$ and certain Fourier coefficients of the derivative of a metaplectic Eisenstein series (Theorem 9.1). This is analogous to Theorem 14.11 of [15] and Theorem 9.2 of [16]. We content ourselves here with pointing out one essential difference with the case of good reduction treated in [15]. In that case, the choice of the local component $\Phi_{p}$ of the function occurring in the Eisenstein series is canonical and it is in fact standard in the sense that its restriction to the maximal compact subgroup of $M p_{2, \mathbb{Q}_{p}}$ is independent of the complex parameter $s$. In our case, the choice of $\Phi_{p}$, given in Corollary 7.4, is no longer canonical; rather, we are able to single out a whole class of functions for which the main identity holds, and these functions are definitely not standard. It seems quite likely that among those functions there are preferred choices, namely those that match up the Eisenstein series with $L$-functions via the doubling method, but this will not concern us here.

Let us give a brief description of the contents of the various sections. Section 1 contains recollections about the Drinfeld moduli space and introduces our terminology (esp. ordinary special and superspecial points). In section 2 we use the building to determine the point set of the special fibre of a special cycle (resp. the intersection of two of them). In section 3 we use the Genestier equations to determine the local structure of a special cycle (multiplicities of vertical components, occurrence of embedded components, etc.). In section 4 we explain the kind of 
intersection theory we are using. In section 5 we construct a global resolution of the structure sheaves of the special cycles and prove the above-mentioned invariance property of the intersection numbers. Section 6 contains the calculation of the intersection number in the diagonalized case. In section 7 we review the results of Myers, [18], and Yang, [20], and establish the connection with representation densities. In section 8 we pose the global intersection problem for cycles on models of Shimura curves over $\mathbb{Z}_{p}$ and relate it to the local theory. The final section 9 gives the relation, extending that of $[\mathbf{1 5}]$, between the intersection numbers and special values of the derivatives of Fourier coefficients of Eisenstein series.

In conclusion we wish to point out again that one of the main ingredients of this paper is due to Genestier [7]; regrettably, he did not pursue the further implications of his idea. We also thank T. Yang for communicating to us his results on representation densities at an early stage of this project. This work was begun at the University of Cologne in August 1997 and continued at the University of Maryland in March 1998. We thank both institutions for their hospitality and the NSF and the DFG for their support.

\section{Contents}

1. The Drinfeld moduli space and the p-adic upper half plane

2. Special cycles and their support in the special fiber

3. Local equations for special cycles

4. Intersection calculus of special cycles

5. An invariance property of intersection numbers

6. Computation of intersection numbers

7. Intersection numbers and representation densities

8. Intersection numbers on Shimura curves

9. Intersection numbers and Fourier coefficients 
Pictures when $\operatorname{dim} Z(j)=1$, for $q(j)=\varepsilon p^{\alpha}$

$\alpha \geq 0$ even, $\chi(\varepsilon)=-1$

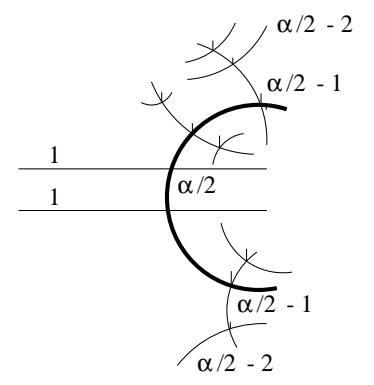

$\alpha \geq 2$ even, $\chi(\varepsilon)=1$

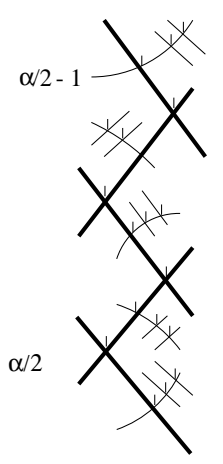

$\alpha \geq 0$ odd

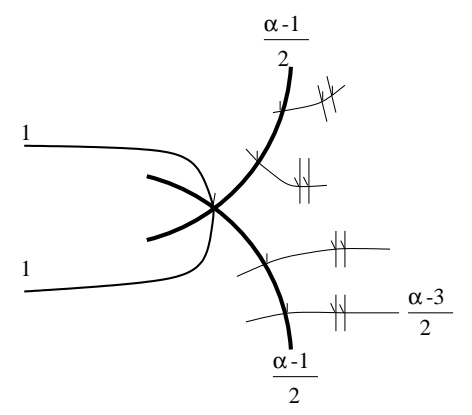

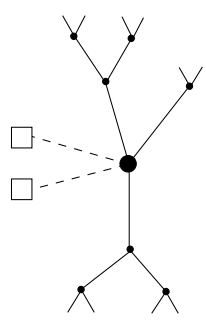
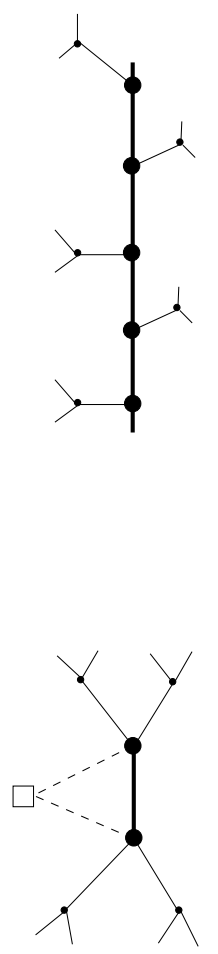

Explanation: On the left the irreducible components of the special cycles are depicted; on the right the dual graph is given. The "central" vertical irreducible components ( 1 in the first case, $\infty$ in the second case, 2 in the third case) are in bold face. They occur with multiplicity $\left[\frac{\alpha}{2}\right]$ in $Z(j)$. The other vertical components have multiplicity $\left[\frac{\alpha}{2}\right]-i$, where $i$ is the "distance" from a central component. Also the horizontal components are given; they are indicated by a box on the right ( 2 in the first case, none in the second case, 1 in the third case). The little barbs indicate embedded components. 


\section{Notation.}

The following notation will be used in the local part of this paper (sections 1-7):

$k$ algebraically closed field of characteristic $p>2$

$W=W(k)$ the ring of Witt vectors of $k$ with its Frobenius automorphism $\sigma$.

$W_{\mathbb{Q}}$ the fraction field of $W$.

$\mathbb{Z}_{p^{2}}=W\left(\mathbb{F}_{p^{2}}\right)$ where $\mathbb{F}_{p^{2}}=\left\{x \in k ; x^{p^{2}}=1\right\}$.

$B$ a quaternion division algebra over $\mathbb{Q}_{p}$.

$O_{B}$ the ring of integers which we identify with

$$
O_{B}=\mathbb{Z}_{p^{2}}[\Pi] / \Pi^{2}-p, \Pi a=a^{\sigma} \Pi \quad\left(a \in \mathbb{Z}_{p^{2}}\right) .
$$

$\mathcal{B}$ the Bruhat-Tits building of $P G L_{2}\left(\mathbb{Q}_{p}\right)$.

$\chi$ the quadratic residue character on $\mathbb{Z}_{p}^{\times}$resp. $\mathbb{F}_{p}^{\times}$.

\section{$\S 1$. The Drinfeld moduli space and the $p$-adic upper half plane.}

In this section we recall some facts from Drinfeld's paper [5], cf. also [2], [6], [19]. A special formal (s.f.) $O_{B}$-module over a $W$-scheme $S$ is a $p$-divisible formal group $X$ of height 4 , with an $O_{B}$-action $\iota: O_{B} \rightarrow \operatorname{End}_{S}(X)$ such that the induced $\mathbb{Z}_{p^{2}} \otimes \mathcal{O}_{S}$-module Lie $X$ is, locally on $S$, free of rank 1 . Let us fix once and for all a s.f. $O_{B}$-module $\mathbb{X}$ over Spec $k$. It is unique up to $O_{B}$-linear isogeny, and $\operatorname{End}_{O_{B}}^{0}(\mathbb{X}) \simeq M_{2}\left(\mathbb{Q}_{p}\right)$. Let us consider the following functor $\mathcal{M}$ on the category Nilp of $W$-schemes $S$ such that $p$ is locally nilpotent in $\mathcal{O}_{S}$. The $S$-valued points of $\mathcal{M}$ are the isomorphism classes of pairs $(X, \varrho)$ consisting of a s.f. $O_{B}-$ module $X$ over $S$ and a quasi-isogeny of height zero,

$$
\varrho: \mathbb{X} \times_{\operatorname{Spec} k} \bar{S} \rightarrow X \times_{S} \bar{S} .
$$

Here $\bar{S}=S \times$ Spec $W$ Spec $k$. We consider

$$
G\left(\mathbb{Q}_{p}\right)^{0}=\left\{g \in G L_{2}\left(\mathbb{Q}_{p}\right) ; \operatorname{ord} \operatorname{det} g=0\right\} .
$$

Then, after choosing an identification $\operatorname{End}_{O_{B}}^{0}(\mathbb{X})=M_{2}\left(\mathbb{Q}_{p}\right)$, the group $G\left(\mathbb{Q}_{p}\right)^{0}$ acts to the left on $\mathcal{M}$, via

$$
g:(X, \varrho) \mapsto\left(X, \varrho \circ g^{-1}\right)
$$

According to Drinfeld, this functor $\mathcal{M}$ is representable by $\hat{\Omega} \times{ }_{\operatorname{Spf}} \mathbb{Z}_{p} \operatorname{Spf} W$. Here $\hat{\Omega}=\hat{\Omega}_{\mathbb{Q}_{p}}^{2}$ is the formal model of the $p$-adic upper half plane associated to the local field $\mathbb{Q}_{p}$ that was introduced by Deligne (comp. [2], chap. 1). The isomorphism

$$
\mathcal{M} \rightarrow \hat{\Omega} \times{ }_{\operatorname{Spf} \mathbb{Z}_{p}} \operatorname{Spf} W
$$


is equivariant for the action of $G\left(\mathbb{Q}_{p}\right)^{0}$, for a suitable identification $\operatorname{End}_{O_{B}}^{0}(\mathbb{X})=$ $M_{2}\left(\mathbb{Q}_{p}\right)$. The group $G\left(\mathbb{Q}_{p}\right)^{0}$ acts on the RHS via the natural action of $P G L_{2}\left(\mathbb{Q}_{p}\right)$ on $\hat{\Omega}$.

We need to describe some features of $\hat{\Omega}$, comp. [2], chap. 1 . We denote by $\mathcal{B}=\mathcal{B}\left(P G L_{2}\left(\mathbb{Q}_{p}\right)\right)$ the Bruhat-Tits building of $P G L_{2}\left(\mathbb{Q}_{p}\right)$. The formal scheme $\hat{\Omega}$ is obtained by glueing open formal subschemes $\hat{\Omega}_{\Delta}$ where $\Delta$ runs over the simplices of $\mathcal{B}$, and

$$
\hat{\Omega}_{\Delta} \cap \hat{\Omega}_{\Delta^{\prime}}= \begin{cases}\hat{\Omega}_{\Delta \cap \Delta^{\prime}} & \text { if } \Delta \cap \Delta^{\prime} \text { is a simplex } \\ \emptyset & \text { if } \Delta \cap \Delta^{\prime}=\emptyset .\end{cases}
$$

For the action of $P G L_{2}\left(\mathbb{Q}_{p}\right)$ on $\hat{\Omega}$ we have

$$
g \hat{\Omega}_{\Delta}=\hat{\Omega}_{g \Delta} .
$$

We now describe the open charts in detail. Because of (1.4) it will suffice to do this for $\Delta=$ the standard vertex and for $\Delta=$ the standard edge.

Let $\Delta=\left[\Lambda_{0}\right]$ be the homothety class of the standard lattice

$$
\Lambda_{0}=\left[e_{1}, e_{2}\right] .
$$

Here $\left[e_{1}, e_{2}\right]$ denotes the $\mathbb{Z}_{p}$-span of the standard basis of $\mathbb{Q}_{p}^{2}$. Then

$$
\hat{\Omega}_{\left[\Lambda_{0}\right]}=\left(\mathbb{P}\left(\Lambda_{0}\right)-\mathbb{P}\left(\Lambda_{0}\right)\left(\mathbb{F}_{p}\right)\right)^{\wedge} .
$$

Here $\mathbb{P}\left(\Lambda_{0}\right) \simeq \mathbb{P}_{\mathbb{Z}_{p}}^{1}$ denotes the relative projective line over $\mathbb{Z}_{p}$ associated to $\Lambda_{0}$ and the "hat" indicates the completion along the special fibre. If we use $e_{1}, e_{2}$ to identify $\mathbb{P}\left(\Lambda_{0}\right)$ with $\mathbb{P}_{\mathbb{Z}_{p}}^{1}$, we have

$$
\hat{\Omega}_{\left[\Lambda_{0}\right]}=\operatorname{Spf} \mathbb{Z}_{p}\left[T,\left(T^{p}-T\right)^{-1}\right]^{\wedge},
$$

where $T=X_{0} / X_{1}$ in terms of the canonical coordinates on $\mathbb{P}_{\mathbb{Z}_{p}}^{1}$. The subgroup $G L_{2}\left(\mathbb{Z}_{p}\right)$ preserves $\Lambda_{0}$ and hence acts on $\hat{\Omega}_{\left[\Lambda_{0}\right]}$. The action of $g$ on $\mathbb{P}\left(\Lambda_{0}\right)$ is induced by the automorphism $g: \Lambda_{0} \rightarrow \Lambda_{0}$. For the left action $f \mapsto g_{*}^{-1}(f)$ of $G L_{2}\left(\mathbb{Z}_{p}\right)$ on the ring of holomorphic functions on $\hat{\Omega}_{\left[\Lambda_{0}\right]}$ we therefore have $g_{*}^{-1}:\left(X_{0}, X_{1}\right) \mapsto\left(\operatorname{det}(g)^{-1}\left(d X_{0}-c X_{1}\right), \operatorname{det}(g)^{-1}\left(-b X_{0}+a X_{1}\right)\right), g=\left(\begin{array}{ll}a & b \\ c & d\end{array}\right)$. 
In terms of the coordinate $T$ the action $g_{*}^{-1}$ is therefore given by

$$
g_{*}^{-1}: T \mapsto \frac{d T-c}{-b T+a} \quad, \quad g=\left(\begin{array}{cc}
a & b \\
c & d
\end{array}\right)
$$

(homography associated to ${ }^{t} g^{-1}$ ).

Next, let $\Delta_{0}=\left(\left[\Lambda_{0}\right],\left[\Lambda_{1}\right]\right)$ be the edge corresponding to

$$
\Lambda_{0}=\left[e_{1}, e_{2}\right], \Lambda_{1}=\left[p e_{1}, e_{2}\right] .
$$

In this case we have an identification

$$
\hat{\Omega}_{\Delta_{0}}=\operatorname{Spf} \mathbb{Z}_{p}\left[T_{0}, T_{1},\left(1-T_{0}^{p-1}\right)^{-1},\left(1-T_{1}^{p-1}\right)^{-1}\right]^{\wedge} /\left(T_{0} T_{1}-p\right)
$$

The action of the Iwahori subgroup

$$
\left(\begin{array}{ll}
\mathbb{Z}_{p}^{\times} & p \mathbb{Z}_{p} \\
\mathbb{Z}_{p} & \mathbb{Z}_{p}^{\times}
\end{array}\right)
$$

is given by

$$
g_{*}^{-1}: T_{0} \mapsto \frac{d T_{0}-p c}{-b_{0} T_{0}+a} \quad, \quad T_{1} \mapsto \frac{a T_{1}-p b_{0}}{-c T_{1}+d}
$$

for

$$
g=\left(\begin{array}{ll}
a & b \\
c & d
\end{array}\right)=\left(\begin{array}{cc}
a & p b_{0} \\
c & d
\end{array}\right)
$$

The open immersion $\hat{\Omega}_{\left[\Lambda_{0}\right]} \rightarrow \hat{\Omega}_{\Delta_{0}}$ is induced by the open immersion

$$
\operatorname{Spf} \mathbb{Z}_{p}\left[T, T^{-1}\right]^{\wedge} \rightarrow \operatorname{Spf}\left(\mathbb{Z}_{p}\left[T_{0}, T_{1}\right] / T_{0} T_{1}-p\right)^{\wedge}
$$

induced by

$$
T_{1} \mapsto T^{-1} \quad, \quad T_{0} \mapsto p \cdot T
$$

It is easy to check that this morphism is indeed equivariant for the action of the Iwahori subgroup (1.11).

The special fibre of $\hat{\Omega}$ is a union of projective lines parametrized by the vertices in $\mathcal{B}$. More precisely, $\mathcal{B}$ can be identified with the dual graph of the special fibre, 
compatibly with the action of $P G L_{2}\left(\mathbb{Q}_{p}\right)$. Let $[\Lambda]$ be a vertex with corresponding projective line $\mathbb{P}_{[\Lambda]}$. Then $\mathbb{P}_{[\Lambda]}$ may be identified with

$$
\mathbb{P}_{[\Lambda]}=\mathbb{P}(\Lambda / p \Lambda)
$$

where $\Lambda$ is any lattice in the homothety class $[\Lambda]$, and

$$
\hat{\Omega}_{[\Lambda]} \times_{\operatorname{Spf} \mathbb{Z}_{p}} \operatorname{Spec} \mathbb{F}_{p}=\mathbb{P}_{[\Lambda]}-\mathbb{P}_{[\Lambda]}\left(\mathbb{F}_{p}\right)
$$

If $\Delta=\left([\Lambda],\left[\Lambda^{\prime}\right]\right)$ is an edge, we denote the corresponding point in the special fibre by $p t_{\Delta}$,

$$
p t_{\left([\Lambda],\left[\Lambda^{\prime}\right]\right)}=\mathbb{P}_{[\Lambda]} \cap \mathbb{P}_{\left[\Lambda^{\prime}\right]}=\hat{\Omega}_{\Delta}\left(\mathbb{F}_{p}\right)
$$

We shall also need to know how the Drinfeld isomorphism (1.2) looks on the set of closed points. Let $(X, \varrho) \in \mathcal{M}(k)$ and let $(M, F, V)$ be the covariant Dieudonné module of $X$. It is a free $W$-module of rank 4 . From the action of $\mathbb{Z}_{p^{2}}$ on $X$ we obtain a $\mathbb{Z} / 2$-grading,

$$
M=M_{0} \oplus M_{1}
$$

and $F, V$ and $\iota(\Pi)$ are all homogeneous of degree 1 . Since Lie $X=M / V M$ and by the condition on $X$ to be special, we have inclusions with index 1 of free $W$-modules of rank 2 ,

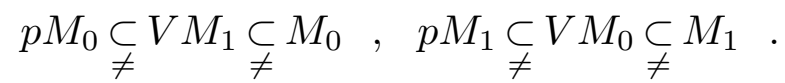

An index $i \in \mathbb{Z} / 2$ is called critical, if

$$
V M_{i}=\Pi M_{i}
$$

Since $\operatorname{Lie}(\iota(\Pi))^{2}=0$ and $\operatorname{dim}_{k} M_{0} / V M_{1}=\operatorname{dim}_{k} M_{1} / V M_{0}=1$, there always exists $i$ with $\Pi M_{i} \subset V M_{i}$. Since both modules have index 1 in $M_{i+1}$, it follows that $i$ is critical. Hence there always exists a critical index.

If $i$ is a critical index, $V^{-1} \Pi$ is a $\sigma$-linear automorphism of $M_{i}$. If we set $\eta_{i}=M_{i}^{V^{-1} \Pi}$, then $\eta_{i}$ is a $\mathbb{Z}_{p}$-module with

$$
M_{i}=\eta_{i} \otimes_{\mathbb{Z}_{p}} W
$$

Recall our fixed s.f. $O_{B}$-module $\mathbb{X}$. We may choose $\mathbb{X}$ in its isogeny class so that 0 and 1 are critical. Let

$$
M^{0}=M_{0}^{0} \oplus M_{1}^{0}, \quad \eta_{i}^{0}=M_{i}^{0^{V^{-1} \Pi}}
$$


be the Dieudonné module of $\mathbb{X}$ and let us fix an isomorphism

$$
U:=\eta_{0}^{0} \otimes_{\mathbb{Z}_{p}} \mathbb{Q}_{p} \simeq \mathbb{Q}_{p}^{2}
$$

We can now describe the Drinfeld isomorphism on $\mathcal{M}(k)$. Let $(X, \varrho) \in \mathcal{M}(k)$ and let $M$ be the Dieudonné module of $X$. If 0 is critical we have the $\mathbb{Z}_{p}$-module $\eta_{0}$ and $\varrho$ defines an isomorphism

$$
\eta_{0} \otimes \mathbb{Q}_{p} \rightarrow U=\mathbb{Q}_{p}^{2}
$$

Let $\Lambda_{0}$ be the image of $\eta_{0}$ under (1.23). Then $\Lambda_{0}$ is a lattice in $\mathbb{Q}_{p}^{2}$ which (since the height of $\varrho$ is zero) is of the same volume as $\mathbb{Z}_{p}^{2}$. The point $(X, \varrho)$ is then mapped to the point of $\mathbb{P}_{\left[\Lambda_{0}\right]}(k)$ corresponding to the line

$$
\ell=V M_{1} / p M_{0} \subset M_{0} / p M_{0}=\Lambda_{0} \otimes_{\mathbb{Z}_{p}} k
$$

If 1 is critical, $\varrho$ defines an isomorphism

$$
\eta_{1} \otimes \mathbb{Q}_{p} \rightarrow \eta_{1}^{0} \otimes \mathbb{Q}_{p}
$$

The action of $\Pi$ identifies $\eta_{1}^{0} \otimes \mathbb{Q}_{p}$ with $U$. Hence we obtain a lattice $\Lambda_{1}$ in $\mathbb{Q}_{p}^{2}$, of the same volume as $p \mathbb{Z}_{p} \oplus \mathbb{Z}_{p}$. The point $(X, \varrho)$ is mapped to the point of $\mathbb{P}_{\left[\Lambda_{1}\right]}(k)$ corresponding to the line

$$
\ell=V M_{0} / p M_{1} \subset M_{1} / p M_{1}=\Lambda_{1} \otimes_{\mathbb{Z}_{p}} k
$$

If both 0 and 1 are critical, both procedures above are applicable and the lattices $\Lambda_{1} \subset \Lambda_{0}$ define an edge $\Delta=\left(\left[\Lambda_{0}\right],\left[\Lambda_{1}\right]\right)$ in $\mathcal{B}$. In this case the line $V M_{1} / p M_{0}$ in $\mathbb{P}_{\left[\Lambda_{0}\right]}$ and $V M_{0} / p M_{1}$ in $\mathbb{P}_{\left[\Lambda_{1}\right]}$ define the same point of $\hat{\Omega}(k)$, namely $p t_{\Delta}$. We thus see that the set of irreducible components of $\hat{\Omega} \otimes k$ can be partitioned into two subsets: on the irreducible components corresponding to even lattice classes the index 0 is critical and on the irreducible components corresponding to odd lattice classes the index 1 is critical.

We shall use the following terminology.

Definition 1.1. A point $(X, \varrho) \in \mathcal{M}(k)$ is called superspecial if both indices 0,1 are critical ordinary if only one index is critical special if $V^{2} M=p M$.

Obviously, a superspecial point is special since in this case $V M=\Pi M$. Assume that $(X, \varrho)$ is ordinary and let e.g. 0 be the unique critical index,

$$
V M_{0}=\Pi M_{0} .
$$


Since $V^{2} M_{0}=p M_{0}$, we see that $(X, \varrho)$ is special if and only if

$$
V^{2} M_{1}=p M_{1}, \text { i.e. }\left(V^{-1} \Pi\right)^{2} V M_{1}=V M_{1}
$$

Recalling that $V M_{1} / p M_{0} \subset M_{0} / p M_{0}$ is the line in $\mathbb{P}_{\left[\Lambda_{0}\right]}$ associated to $M$, we obtain the following characterization.

Proposition 1.2. Let $\mathbb{P}_{[\Lambda]} \subset \hat{\Omega} \otimes_{\mathbb{Z}_{p}} k$ be the irreducible component corresponding to the vertex $[\Lambda]$ in $\mathcal{B}$. Then a point $x \in \mathbb{P}_{[\Lambda]}(k)$ is

superspecial iff $x \in \mathbb{P}_{[\Lambda]}\left(\mathbb{F}_{p}\right)$

special iff $x \in \mathbb{P}_{[\Lambda]}\left(\mathbb{F}_{p^{2}}\right)$.

Remark 1.3. Let $(X, \varrho) \in \mathcal{M}(k)$ be special. Then we may find a $W$-basis of its Dieudonné module with

$$
e_{3}=V e_{1}, e_{4}=V e_{2}, p e_{1}=V e_{3}, p e_{2}=V e_{4}
$$

Hence $X$ is isomorphic to the product of the $p$-divisible group of a supersingular elliptic curve with itself. The converse also holds.

\section{$\S 2$. Special cycles and their support in the special fibre.}

Recall our fixed s.f. $O_{B}$-module $\mathbb{X}$ over $k$. We introduce

$$
V=\left\{j \in \operatorname{End}_{O_{B}}^{0}(\mathbb{X}) ; \operatorname{tr}^{0}(j)=0\right\}
$$

We will always identify $j \in \operatorname{End}_{O_{B}}^{0}(\mathbb{X})$ with its image under the identifications (cf.

$$
\operatorname{End}_{O_{B}}^{0}(\mathbb{X})=\operatorname{End}(U)=M_{2}\left(\mathbb{Q}_{p}\right)
$$

Then $V$ is equipped with a quadratic form given by squaring,

$$
j^{2}=q(j) \cdot \mathrm{id}, \quad q(j) \in \mathbb{Q}_{p} .
$$

We note that

$$
q(j)=-\operatorname{det}(j)
$$

The elements of $V$ will be called special endomorphisms. 
Definition 2.1. Let $j \in V$ be a special endomorphism with $q(j) \neq 0$. The special cycle $Z(j)$ associated to $j$ is the closed formal subscheme of $\mathcal{M}$ consisting of all points $(X, \varrho)$ such that $\varrho \circ j \circ \varrho^{-1}$ lifts to an endomorphism of $X$.

Let $(X, \varrho) \in \mathcal{M}(S)$. Then $\lambda=\varrho \circ j \circ \varrho^{-1}$ is a quasi-isogeny of $X \times_{S} \bar{S}$. By the rigidity property of quasi-isogenies ([5]) we may also consider $\lambda$ as a quasi-isogeny of $X$. The locus in $S$ where $\lambda$ is an isogeny is a closed subscheme $S^{\prime}$ of $S$ ([19], prop. 2.9). The closed formal subscheme $Z(j)$ of $\mathcal{M}$ is characterized by

$$
S^{\prime}=Z(j) \times_{\mathcal{M}} S
$$

In this section we will study the point set $Z(j)(k)$ of special cycles and their intersection properties.

Proposition 2.1. Let $[\Lambda]$ be a vertex in $\mathcal{B}$ and let $\Lambda \subset U=\mathbb{Q}_{p}^{2}$ be a lattice in its homothety class. Then

$$
\mathbb{P}_{[\Lambda]} \cap Z(j) \neq \emptyset \quad \text { iff } j(\Lambda) \subset \Lambda .
$$

In particular, if $Z(j) \neq \emptyset$ then $q(j) \in \mathbb{Z}_{p}$.

We will see in Corollary 2.5 that conversely, if $q(j) \in \mathbb{Z}_{p}$, then $Z(j) \neq \emptyset$.

Proof. Suppose e.g. that $[\Lambda]$ is even and let $x \in \mathbb{P}_{[\Lambda]}(k)$. Then $x$ corresponds under the Drinfeld isomorphism to $(X, \varrho)$ where 0 is a critical index and with Dieudonné module $M=M_{0} \oplus M_{1}$, where

$$
M_{0}=\Lambda \otimes_{\mathbb{Z}_{p}} W \quad, \quad V M_{1} / p M_{0}=\ell_{x} \subset \Lambda \otimes k .
$$

Then $x$ lies in $Z(j)$ iff $j \otimes_{\mathbb{Q}_{p}} \operatorname{id}_{W_{\mathbb{Q}}}$ preserves the $W$-lattices $M_{0}$ and $V M_{1}$ in $U \otimes_{\mathbb{Q}_{p}} W_{\mathbb{Q}}$. This proves one implication. Assume now that $j(\Lambda) \subset \Lambda$, i.e. $j\left(M_{0}\right) \subset M_{0}$. Since $k$ is algebraically closed there exists a line $\ell \subset \Lambda \otimes_{\mathbb{Z}_{p}} k$ stable under the endomorphism induced by $j$. The corresponding point $x \in \mathbb{P}_{[\Lambda]}(k)$ is associated to $(X, \varrho)$ with $M=M_{0} \oplus M_{1}$ where

$$
j\left(V M_{1} / p M_{0}\right) \subset V M_{1} / p M_{0} \text {, i.e., } j\left(V M_{1}\right) \subset V M_{1} .
$$

Hence $x \in Z(j)$.

The last assertion follows by taking a $\mathbb{Z}_{p}$-basis of a lattice $\Lambda$ with $\mathbb{P}_{[\Lambda]} \cap Z(j) \neq \emptyset$ and using it to calculate $\operatorname{det}(j)=-q(j)$. 
From now on we will always assume that $q(j) \in \mathbb{Z}_{p}-\{0\}$. We next do a local analysis of $Z(j)$ along a line $\mathbb{P}_{[\Lambda]}$ which intersects $Z(j)$. Let us fix a lattice $\Lambda \subset U$ with $j(\Lambda) \subset \Lambda$. We use the notation

$$
\operatorname{red}_{\Lambda}(j) \in \operatorname{End}(\Lambda / p \Lambda)
$$

for the induced endomorphism.

Proposition 2.2. With the notation introduced above, there are the following possibilities.

(i) $\operatorname{rk}\left(\operatorname{red}_{\Lambda}(j)\right)=2$. Then $q(j)=\varepsilon \in \mathbb{Z}_{p}^{\times}$and $\operatorname{red}(j)$ preserves precisely two lines in $\mathbb{P}_{[\Lambda]}(k)$. The corresponding points are both superspecial, if $\chi(\varepsilon)=1$ and both ordinary special, if $\chi(\varepsilon)=-1$.

(ii) $\operatorname{rk}\left(\operatorname{red}_{\Lambda}(j)\right)=1$. Then $\operatorname{ord} q(j) \geq 1$ and $\operatorname{red}_{\Lambda}(j)$ is a nilpotent endomorphism. The line

$$
\ell=\operatorname{Ker}_{\operatorname{red}_{\Lambda}}(j)=\operatorname{Im}_{\operatorname{red}_{\Lambda}}(j)
$$

is the unique line stable under $\operatorname{red}_{\Lambda}(j)$. The corresponding point of $\mathbb{P}_{\Lambda}$ is superspecial.

(iii) $\operatorname{red}_{\Lambda}(j)=0$. Then ord $q(j) \geq 2$ and all lines $\ell \in \mathbb{P}_{[\Lambda]}(k)$ are stable under $\operatorname{red}_{\Lambda}(j)$, i.e. $\mathbb{P}_{[\Lambda]} \subset Z(j)$.

Proof. Let us prove (i). Since $\operatorname{red}_{\Lambda}(j)$ is a traceless automorphism it has two distinct eigen lines. The characteristic polynomial of $\operatorname{red}_{\Lambda}(j)$ has the form

$$
X^{2}-q(j) \bmod p
$$

since $q(j)=-\operatorname{det}(j)$, and the determinant may be calculated using a basis of $\Lambda$. The assertion follows. The other assertions are proved in a similar way.

Corollary 2.3. $Z(j)(k)$ consists only of isolated points if and only if ord $q(j) \leq 1$.

Proof. If $\mathbb{P}_{[\Lambda]} \subset Z(j)$ then $\operatorname{red}_{\Lambda}(j)=0$ and $\operatorname{ord} q(j) \geq 2$. Conversely, let ord $q(j) \geq 2$. By Corollary 2.5 below there exists $\Lambda$ with $\mathbb{P}_{[\Lambda]} \cap Z(j) \neq \emptyset$. Then $\operatorname{red}_{\Lambda}(j)$ cannot have full rank because otherwise ord $q(j)=0$. Therefore, if $\mathbb{P}_{[\Lambda]} \not \subset Z(j)$ we are in case (ii) of the previous proposition. Let $\Lambda^{\prime}$ be the lattice neighbour of $\Lambda$ corresponding to the intersection point of $\mathbb{P}_{[\Lambda]}$ with $Z(j)$, i.e. to the line $\operatorname{Im}_{\operatorname{red}}(j)$ in $\Lambda / p \Lambda$. Then

$$
\Lambda^{\prime}=j(\Lambda)+p \Lambda
$$


But then

$$
\begin{aligned}
j\left(\Lambda^{\prime}\right) & =j^{2}(\Lambda)+p j(\Lambda) \\
& =p^{\alpha} \Lambda+p j(\Lambda), \quad \alpha \geq 2 \\
& =p\left(j(\Lambda)+p^{\alpha-1} \Lambda\right) \\
& \subset p \Lambda^{\prime} .
\end{aligned}
$$

Hence $\operatorname{red}_{\Lambda^{\prime}}(j)=0$ and $\mathbb{P}_{\left[\Lambda^{\prime}\right]} \subset Z(j)$.

We next will get a global overview of the lattices $\Lambda$ which satisfy the criterion of Proposition 2.1, i.e., of the set

$$
\mathcal{T}(j)=\left\{[\Lambda] \in \mathcal{B} ; \quad \mathbb{P}_{[\Lambda]} \cap Z(j) \neq \emptyset\right\}
$$

Lemma 2.4. Let $j \in G L_{2}\left(\mathbb{Q}_{p}\right)$. Let $\Lambda \subset \mathbb{Q}_{p}^{2}$ be a lattice. The following conditions are equivalent:

(i) $j(\Lambda) \subset \Lambda$

(ii) Let $[\Lambda] \in \mathcal{B}$ be the vertex corresponding to $\Lambda$. Then

$$
d([\Lambda],[j(\Lambda)]) \leq \operatorname{ord} \operatorname{det} j
$$

Here $d$ denotes the distance in the building.

If $\operatorname{tr}(j)=0$, these conditions are also equivalent to

(iii) $d\left([\Lambda], \mathcal{B}^{j}\right) \leq \frac{1}{2} \cdot \operatorname{ord} \operatorname{det} j$.

Here $\mathcal{B}^{j}$ denotes the fixed point set of $j$ in $\mathcal{B}$.

Proof. Put $\alpha=$ ord det $j$. Let $(e, f) \in \mathbb{Z}^{2}, e \geq f$, be the elementary divisors for the lattice pair $\Lambda, j(\Lambda)$. Then $e+f=\alpha$ and $d([\Lambda],[j(\Lambda)])=e-f$. On the other hand

$$
j(\Lambda) \subset \Lambda \Longleftrightarrow f \geq 0 \Longleftrightarrow e-f \leq \alpha
$$

This shows the equivalence of (i) and (ii). For (iii) we note that if $\operatorname{tr}(j)=0$, then $j$ induces an involution of $\mathcal{B}$. The unique geodesic from $[\Lambda]$ to $[j(\Lambda)]$ consists of the geodesic from $[\Lambda]$ to $\mathcal{B}^{j}$ and its image under $j$ which is the geodesic from $[j(\Lambda)]$ to $\mathcal{B}^{j}$.

Remark: This observation appears already in $[\mathbf{1 4}]$ where it is attributed to Tate. 
Corollary 2.5. Let $j \in V$ with $q(j)=\varepsilon \cdot p^{\alpha}$, where $\varepsilon \in \mathbb{Z}_{p}^{\times}, \alpha \geq 0$. The set $\mathcal{T}(j)$ (cf. (2.10)) is as follows:

(i) If $\alpha$ is even and $\chi(\varepsilon)=-1$, then $\mathcal{T}(j)$ is a ball of radius $\alpha / 2$ around the unique vertex $\left[\Lambda_{0}\right]$ fixed by $j$.

(ii) If $\alpha$ is even and $\chi(\varepsilon)=1$, then $\mathcal{T}(j)$ is a tube of width $\alpha / 2$ around the apartment fixed by $j$.

(iii) If $\alpha$ is odd and $\chi(\varepsilon)$ arbitrary, then $\mathcal{T}(j)$ is a ball of radius $\alpha / 2$ around the unique fixed point of $j$, which is the midpoint of an edge.

In particular, in all cases $\mathcal{T}(j) \neq \emptyset$.

Proof. By the preceding lemma we only have to determine $\mathcal{B}^{j}$. In cases (i) and (ii) and after replacing $j$ by a scalar multiple we may assume that $j^{2}=\varepsilon \cdot \mathrm{id}$. In case (i) we may find a basis $e_{1}, e_{2}$ of $\mathbb{Q}_{p}^{2}$ such that $j$ has the matrix

$$
j=\left(\begin{array}{ll}
0 & \varepsilon \\
1 & 0
\end{array}\right)
$$

In this case $[\Lambda]$ with $\Lambda=\left[e_{1}, e_{2}\right]$ is the unique vertex fixed by $j\left(\operatorname{red}_{\Lambda}(j)\right.$ fixes no $\mathbb{F}_{p}$-rational line in $\Lambda / p \Lambda$ ). In case (ii) we may assume that $j^{2}=$ id and can find a basis $e_{1}, e_{2}$ of $\mathbb{Q}_{p}^{2}$ such that $j$ has the matrix

$$
j=\left(\begin{array}{cc}
1 & 0 \\
0 & -1
\end{array}\right) \text {. }
$$

In this case the fixed point set is given by the apartment with vertices $\left[\Lambda_{i}\right]$,

$$
\Lambda_{i}=\left[p^{i} e_{1}, e_{2}\right], \quad i \in \mathbb{Z} .
$$

The case (iii) is also easy and is left to the reader.

Corollary 2.6. In the cases when $Z(j)(k)$ is a set of isolated points (cf. Corollary 2.3), this point set is of the following form.

(i) $q(j)=\varepsilon \in \mathbb{Z}_{p}^{\times}, \chi(\varepsilon)=-1$. In this case let $\left[\Lambda_{0}\right]$ be the unique fixed vertex of $j$. Then $Z(j)(k)$ consists of two ordinary special points on $\mathbb{P}_{\left[\Lambda_{0}\right]}$ (namely the two eigen lines of $\left.\operatorname{red}_{\left[\Lambda_{0}\right]}(j)\right)$.

(ii) $q(j)=\varepsilon \in \mathbb{Z}_{p}^{\times}, \chi(\varepsilon)=1$. In this case let (2.13) be the apartment fixed by $j$. Then

$$
Z(j)(k)=\left\{p t_{\left(\left[\Lambda_{i}, \Lambda_{i-1}\right]\right)} ; \quad i \in \mathbb{Z}\right\}
$$

(iii) $q(j)=\varepsilon \cdot p, \chi(\varepsilon)$ arbitrary. Let $\Delta$ be the edge containing the unique midpoint fixed by $j$. Then

$$
Z(j)(k)=\left\{p t_{\Delta}\right\}
$$


Proof. This is simply a combination of the previous corollary with the local analysis of Proposition 2.2.

For the next corollary we need the following slight strengthening of Lemma 2.4. The proof is identical.

Lemma 2.7. Let $j \in G L_{2}\left(\mathbb{Q}_{p}\right)$ with $\operatorname{tr}(j)=0$, and let $q(j)=\varepsilon \cdot p^{\alpha}, \varepsilon \in \mathbb{Z}_{p}^{\times}$. Let $\Lambda \subset \mathbb{Q}_{p}^{2}$ be a lattice. Let

$$
m_{[\Lambda]}(j):=\max \left\{r ; j(\Lambda) \subset p^{r} \Lambda\right\}
$$

Then

$$
m_{[\Lambda]}(j)=\alpha / 2-d\left([\Lambda], \mathcal{B}^{j}\right)
$$

Corollary 2.8. Let $j \in V$ with $q(j)=\varepsilon \cdot p^{\alpha}, \alpha \geq 1$. For all $[\Lambda] \in \mathcal{T}(j)$ not on the boundary of $\mathcal{T}(j)$ we have $\mathbb{P}_{[\Lambda]} \subset Z(j)$. If $[\Lambda]$ is on the boundary of $\mathcal{T}(j)$, i.e. $d\left([\Lambda], \mathcal{B}^{j}\right)=\alpha / 2$, then $\mathbb{P}_{[\Lambda]} \cap Z(j)$ consists of a single superspecial point, namely the one corresponding to the unique neighbouring vertex of $[\Lambda]$ in $\mathcal{T}(j)$.

We next turn to the intersection of two special cycles. Obviously the intersection $Z\left(j, j^{\prime}\right)=Z(j) \cap Z\left(j^{\prime}\right)$ will only depend on the $\mathbb{Z}_{p}$-span $\mathbf{j}$ of $j$ and $j^{\prime}$, which we will assume to be of rank 2 . Let

$$
(,): V \times V \rightarrow \mathbb{Q}_{p}
$$

be the bilinear form corresponding to the quadratic form $q$,

$$
(x, y)=q(x+y)-q(x)-q(y)
$$

Since $p \neq 2$, the restriction of $($,$) to \mathbf{j}$ may be diagonalized. We will always assume that $\mathbf{j}$ is non-degenerate. Then we may choose a $\mathbb{Z}_{p}$-basis $j, j^{\prime}$ of $\mathbf{j}$ such that the restriction of $($,$) to \mathbf{j}$ has matrix

$$
T:=\left(\begin{array}{cc}
q(j) & \frac{1}{2}\left(j, j^{\prime}\right) \\
\frac{1}{2}\left(j, j^{\prime}\right) & q\left(j^{\prime}\right)
\end{array}\right)=\operatorname{diag}\left(\varepsilon_{1} p^{\alpha}, \varepsilon_{2} p^{\beta}\right)
$$

with $\varepsilon_{1}, \varepsilon_{2} \in \mathbb{Z}_{p}^{\times}$, and $\alpha \geq 0, \beta \geq 0$. In particular $j$ and $j^{\prime}$ anticommute,

$$
j j^{\prime}=-j^{\prime} j .
$$


We wish to determine the $k$-rational points of

$$
Z(\mathbf{j})=Z(j) \cap Z\left(j^{\prime}\right)
$$

We introduce

$$
\mathcal{T}(\mathbf{j})=\left\{[\Lambda] \in \mathcal{B} ; \mathbb{P}_{[\Lambda]} \cap Z(\mathbf{j}) \neq \emptyset\right\}
$$

As in the case of a special single endomorphism, we start with a local analysis. Let $\Lambda$ be a lattice such that the corresponding vertex $[\Lambda]$ lies in $\mathcal{T}(\mathbf{j})$. Let

$$
\mathfrak{m}=\operatorname{red}_{\Lambda}(\mathbf{j}) \subset \operatorname{End}(\Lambda / p \Lambda)
$$

Then $\mathfrak{m}$ lies in the subspace of traceless matrices in $\operatorname{End}(\Lambda / p \Lambda) \simeq M_{2}\left(\mathbb{F}_{p}\right)$. Let

$$
(,): \mathfrak{m} \times \mathfrak{m} \rightarrow \mathbb{F}_{p}
$$

be the bilinear form associated to the quadratic form $j \mapsto-\operatorname{det}(j)$. Note that the matrix of this form with respect to the basis $\operatorname{red}_{\Lambda}(j), \operatorname{red}_{\Lambda}\left(j^{\prime}\right)$ is just the reduction modulo $p$ of the matrix $T$ with respect to $j, j^{\prime}$. Let $\operatorname{rkm}$ be the rank of this reduction.

Proposition 2.9. With the previous notation, we have $\mathrm{rk} \mathfrak{m} \leq 1$. Furthermore,

(i) If $\mathrm{rk} \mathfrak{m}=1$ and $\mathfrak{m}$ represents 1 , there are two possibilities

a) $\operatorname{dim} \mathfrak{m}=2$. In this case $\mathbb{P}_{[\Lambda]} \cap Z(\mathbf{j})$ consists of a single superspecial point, which is an isolated point of $Z(\mathbf{j}) \times{ }_{\operatorname{Spf} W} \operatorname{Spec} k$.

b) $\operatorname{dim} \mathfrak{m}=1$. In this case $\mathbb{P}_{[\Lambda]} \cap Z(\mathbf{j})$ consists of two superspecial points, which are isolated points of $Z(\mathbf{j}) \times \operatorname{Spf} W \operatorname{Spec} k$.

(ii) If $\operatorname{rk} \mathfrak{m}=1$ and $\mathfrak{m}$ does not represent 1 , then $\operatorname{dim} \mathfrak{m}=1$ and $\mathbb{P}_{[\Lambda]} \cap Z(\mathbf{j})$ consists of two ordinary special points, which are isolated points of $Z(\mathbf{j}) \times_{\operatorname{Spf}} W$ Spec $k$.

(iii) If $\mathrm{rk} \mathfrak{m}=0$, then $\operatorname{dim} \mathfrak{m} \leq 1$ and there are two possibilities.

a) $\operatorname{dim} \mathfrak{m}=1$. Then $\mathbb{P}_{[\Lambda]} \cap Z(\mathbf{j})$ consists of a single superspecial point. This is an isolated point of $Z(\mathbf{j}) \times_{\operatorname{Spf} W} \operatorname{Spec} k$ iff $p^{2} \chi T$.

b) $\mathfrak{m}=0$. Then $\mathbb{P}_{[\Lambda]} \subset Z(\mathbf{j})$.

Proof. Suppose by contradiction that $\operatorname{rk} \mathfrak{m}=2 . \operatorname{Then}_{\operatorname{red}_{\Lambda}}(j)$ and $\operatorname{red}_{\Lambda}\left(j^{\prime}\right)$ would be traceless invertible linear transformations which anticommute. But then each 
has to interchange the two eigenspaces of the other. But these eigen lines correspond precisely to

$$
\mathbb{P}_{[\Lambda]} \cap Z(j) \text { resp. } \mathbb{P}_{[\Lambda]} \cap Z\left(j^{\prime}\right)
$$

It would follow that $\mathbb{P}_{[\Lambda]} \cap Z(\mathbf{j})=\emptyset$, contrary to our assumption.

Let us prove (i). If $\mathfrak{m}$ represents 1 , we may choose the basis $j, j^{\prime}$ of $\mathbf{j}$ such that

$$
\operatorname{red}_{\Lambda}(j)^{2}=\mathrm{id}, \operatorname{red}_{\Lambda}\left(j^{\prime}\right)^{2}=0
$$

If $\operatorname{dim} \mathfrak{m}=2$, then

$$
\operatorname{Im} \operatorname{red}_{\Lambda}\left(j^{\prime}\right)=\operatorname{Ker} \operatorname{red}_{\Lambda}\left(j^{\prime}\right)
$$

is a one-dimensional subspace of $\Lambda / p \Lambda$ preserved by $\operatorname{red}_{\Lambda}(j)$ since $j j^{\prime}=-j^{\prime} j$. The superspecial point corresponding to this $\mathbb{F}_{p}$-rational point of $\mathbb{P}_{[\Lambda]}$ is the unique point of $\mathbb{P}_{[\Lambda]} \cap Z(\mathbf{j})$. If $\operatorname{dim} \mathfrak{m}=1$, then $\operatorname{red}_{\Lambda}\left(j^{\prime}\right)=0$ and $\mathbb{P}_{[\Lambda]} \cap Z(\mathbf{j})$ consists of the two superspecial points given by the two $\mathbb{F}_{p}$-rational eigenlines of $\operatorname{red}_{\Lambda}(j)$. By corollary 2.3 in both cases we are dealing with isolated points of $Z(j) \times{ }_{\text {Spf } W}$ Spec $k$. Let us now prove (ii). If $\mathrm{rk} \mathfrak{m}=1$ and $\mathfrak{m}$ does not represent 1 we may assume that

$$
\operatorname{red}_{\Lambda}(j)^{2} \in \mathbb{F}_{p}^{\times}-\mathbb{F}_{p}^{\times, 2}
$$

Then $\operatorname{red}_{\Lambda}(j)$ has two non-rational eigen lines. Since $\operatorname{red}_{\Lambda}\left(j^{\prime}\right)$ anticommutes with $\operatorname{red}_{\Lambda}(j)$ it has to take any one of these lines into the other one. Since $\operatorname{red}_{\Lambda}\left(j^{\prime}\right)$ is not invertible, its restriction to at least one of the eigen lines has to be zero. But then $\operatorname{red}_{\Lambda}\left(j^{\prime}\right)$ has to kill both eigenlines since they are not $\mathbb{F}_{p}$-rational. Hence $\operatorname{dim} \mathfrak{m}=1$ and $\mathbb{P}_{[\Lambda]} \cap Z(\mathbf{j})=\mathbb{P}_{[\Lambda]} \cap Z(j)$ consists of the two ordinary special points corresponding to the two eigen lines. These again are isolated points of $Z(j) \times_{\text {Spf } W} \operatorname{Spec} k$.

Finally, let us prove (iii). If $\operatorname{rk} \mathfrak{m}=0$, then $\operatorname{dim} \mathfrak{m} \leq 1$. If $\operatorname{dim} \mathfrak{m}=1$ we may assume that

$$
\operatorname{red}_{\Lambda}(j) \neq 0, \operatorname{red}_{\Lambda}(j)^{2}=0, \operatorname{red}_{\Lambda}\left(j^{\prime}\right)=0 .
$$

In this case $\mathbb{P}_{[\Lambda]} \cap Z(\mathbf{j})=\mathbb{P}_{[\Lambda]} \cap Z(j)$ consists of a single superspecial point corresponding to the $\mathbb{F}_{p}$-rational line

$$
\operatorname{Im} \operatorname{red}_{\Lambda}(j)=\operatorname{Ker} \operatorname{red}_{\Lambda}(j) \subset \Lambda / p \Lambda
$$

If $p^{2} \times T$ then ord $q(j) \leq 1$ and $Z(j) \times_{\operatorname{Spf} W} \operatorname{Spec} k$ consists of isolated points only. If $p^{2} \mid T$, then ord $q(j) \geq 2$ and we put $\Lambda^{\prime}=j(\Lambda)+p \Lambda$, cf. (2.9). Then our superspecial point lies on $\mathbb{P}_{[\Lambda]} \cap \mathbb{P}_{\left[\Lambda^{\prime}\right]}$. But

$$
j^{\prime}(\Lambda) \subset p \Lambda \text { and } j\left(\Lambda^{\prime}\right) \subset p \Lambda^{\prime}
$$


cf. (2.9). Hence

$$
j^{\prime}\left(\Lambda^{\prime}\right)=j^{\prime}(j(\Lambda)+p \Lambda) \subset p(j(\Lambda)+p \Lambda)=p \Lambda^{\prime} .
$$

It follows that $\mathbb{P}_{\left[\Lambda^{\prime}\right]} \subset Z(\mathbf{j})$ and hence our intersection point is not isolated in $Z(\mathbf{j}) \times \times_{\text {Spf } W} \operatorname{Spec} k$.

Finally, if $\mathfrak{m}=(0)$, then obviously all lines in $\mathbb{P}_{[\Lambda]}$ are preserved by $\operatorname{red}_{\Lambda}(j)$, $\forall j \in \mathbf{j}$, i.e. $\mathbb{P}_{[\Lambda]} \subset Z(\mathbf{j})$.

We next turn to a global analysis of $\mathcal{T}(\mathbf{j})$. We obviously have an inclusion

$$
\mathcal{T}(\mathbf{j}) \subset \mathcal{T}(j) \cap \mathcal{T}\left(j^{\prime}\right),
$$

for any set $j, j^{\prime}$ of generators of the $\mathbb{Z}_{p}$-module $\mathbf{j}$. We therefore start by determining $\mathcal{T}(j) \cap \mathcal{T}\left(j^{\prime}\right)$ in the case when $j, j^{\prime}$ diagonalize the bilinear form on $\mathbf{j}$, cf. (2.18). We shall do this according to the following table.

\begin{tabular}{|c|c|c|c|}
\hline$j \backslash j^{\prime}$ & $\begin{array}{c}\beta \text { even } \\
\chi\left(\varepsilon_{2}\right)=-1\end{array}$ & $\begin{array}{c}\beta \text { even } \\
\chi\left(\varepsilon_{2}\right)=1\end{array}$ & $\beta$ odd \\
\hline $\begin{array}{c}\alpha \text { even } \\
\chi\left(\varepsilon_{1}\right)=-1\end{array}$ & & & $\emptyset$ \\
\hline $\begin{array}{c}\alpha \text { even } \\
\chi\left(\varepsilon_{1}\right)=1\end{array}$ & & & \\
\hline$\alpha$ odd & $\emptyset$ & & \\
\hline
\end{tabular}

Remark 2.10. We note that the existence of a basis $j, j^{\prime}$ of the type (2.18) imposes restrictions on the matrix $T$. Indeed, $j$ and $j^{\prime}$ generate a non-commutative $\mathbb{Q}_{p}$ subalgebra of $M_{2}\left(\mathbb{Q}_{p}\right)$ which therefore has to be all of $M_{2}\left(\mathbb{Q}_{p}\right)$. On the other hand, the subalgebra generated by $j$ and $j^{\prime}$ is just the quaternion algebra over $\mathbb{Q}_{p}$ with invariant

$$
\left(\varepsilon_{1} p^{\alpha}, \varepsilon_{2} p^{\beta}\right)_{p} \in \mathbb{Z} / 2
$$

We conclude that

$$
\left(\varepsilon_{1} p^{\alpha}, \varepsilon_{2} p^{\beta}\right)_{p}=\chi(-1)^{\alpha \beta} \cdot \chi\left(\varepsilon_{1}\right)^{\beta} \cdot \chi\left(\varepsilon_{2}\right)^{\alpha}=1 .
$$

This excludes the following cases.

$\alpha$ odd and $\beta$ even and $\chi\left(\varepsilon_{2}\right)=-1$, resp.

$\beta$ odd and $\alpha$ even and $\chi\left(\varepsilon_{1}\right)=-1$. 
$\alpha$ and $\beta$ odd and $\chi\left(-\varepsilon_{1} \varepsilon_{2}\right)=-1$.

Before stating the result, we point out that, since $j$ and $j^{\prime}$ anticommute, each one of the induced automorphisms of $\mathcal{B}$ will preserve the fixed point set of the other. (This remark incidentally gives another reason why the cases (2.28) are excluded: in these cases one fixed point set is a vertex and the other a midpoint.)

Proposition 2.11. Let $j, j^{\prime}$ be a basis of $\mathbf{j}$ such that the matrix of the bilinear form is given by (2.18). The set $\mathcal{T}(j) \cap \mathcal{T}\left(j^{\prime}\right)$ is of the following form.

(i) $\alpha$ and $\beta$ even, $\chi\left(\varepsilon_{1}\right)=\chi\left(\varepsilon_{2}\right)=-1$. In this case $\mathcal{T}(j) \cap \mathcal{T}\left(j^{\prime}\right)$ is the ball with radius $\min (\alpha / 2, \beta / 2)$ around the unique common fixed vertex of $j$ and $j^{\prime}$.

(ii) $\alpha$ and $\beta$ even, $\chi\left(\varepsilon_{1}\right) \neq \chi\left(\varepsilon_{2}\right)$. Suppose e.g. that $\chi\left(\varepsilon_{1}\right)=-1$. Then the unique vertex $\left[\Lambda_{0}\right]$ fixed by $j$ lies on the apartment fixed by $j^{\prime}$. In this case $\mathcal{T}(j) \cap \mathcal{T}\left(j^{\prime}\right)$ is the intersection of the ball with radius $\alpha / 2$ around $\left[\Lambda_{0}\right]$ with the tube of width $\beta / 2$ around the fixed apartment. The case when $\chi\left(\varepsilon_{2}\right)=-1$ is analogous with the roles of $j$ and $j^{\prime}$ interchanged.

(iii) $\alpha$ and $\beta$ even, $\chi\left(\varepsilon_{1}\right)=\chi\left(\varepsilon_{2}\right)=1$. In this case the fixed apartment of $j$ and the fixed apartment of $j^{\prime}$ have a unique vertex in common. The set $\mathcal{T}(j) \cap \mathcal{T}\left(j^{\prime}\right)$ is the intersection of the tube of width $\alpha / 2$ around the fixed apartment of $j$ and the tube of width $\beta / 2$ around the fixed apartment of $j^{\prime}$.

(iv) $\alpha$ odd, $\beta$ even and $\chi\left(\varepsilon_{2}\right)=1$ (resp. $\beta$ odd, $\alpha$ even and $\chi\left(\varepsilon_{1}\right)=1$ ). In this case the midpoint fixed by $j$ lies on the fixed apartment of $j^{\prime}$. The set $\mathcal{T}(j) \cap \mathcal{T}\left(j^{\prime}\right)$ is the intersection of the ball of radius $\alpha / 2$ around the midpoint fixed by $j$ with the tube of width $\beta / 2$ around the apartment fixed by $j^{\prime}$.

(v) $\alpha$ and $\beta$ odd. In this case $j$ and $j^{\prime}$ fix the same midpoint and $\mathcal{T}(j) \cap \mathcal{T}\left(j^{\prime}\right)$ is the ball with radius $\min (\alpha / 2, \beta / 2)$ around it.

Proof. Let us prove the first statement in (iii). After correcting $j$ and $j^{\prime}$ by scalars we may assume that $j^{2}=j^{\prime 2}=\mathrm{id}$. We may choose a basis $e_{1}, e_{2}$ of $\mathbb{Q}_{p}^{2}$ such that $j\left(e_{1}\right)=e_{1}, j\left(e_{2}\right)=-e_{2}$, cf. (2.12). Since $j$ and $j^{\prime}$ anticommute, $j^{\prime}$ has to permute the two eigenlines of $j$, hence $j^{\prime}\left(e_{1}\right)= \pm e_{2}, j\left(e_{2}\right)= \pm e_{1}$. If $\Lambda=\left[e_{1}, e_{2}\right]$ we see that $j^{\prime}$ interchanges the two neighbouring vertices $\left[p^{ \pm 1} e_{1}, e_{2}\right]$ of $[\Lambda]$ in the fixed apartment of $j$, cf. (2.13). Since the common fixed point set of $j$ and $j^{\prime}$ is 
convex, it consists of $[\Lambda]$ only.

The other assertions are equally easy.

Combining this now with our analysis of the special fibres of $Z(j)$ and $Z\left(j^{\prime}\right)$ we obtain the following corollary.

Corollary 2.12. Let $j, j^{\prime}$ be a basis of $\mathbf{j}$ such that the matrix of the bilinear form is given by (2.18). We have an equality $\mathcal{T}(\mathbf{j})=\mathcal{T}(j) \cap \mathcal{T}\left(j^{\prime}\right)$ except when $\alpha=\beta=0$. The latter condition is equivalent to $Z(\mathbf{j})=\emptyset$.

Proof. If $[\Lambda]$ does not lie on the boundary of either $\mathcal{T}(j)$ or $\mathcal{T}\left(j^{\prime}\right)$ we have by Corollary 2.8 that $\mathbb{P}_{[\Lambda]} \subset Z(j)$ or $\mathbb{P}_{[\Lambda]} \subset Z\left(j^{\prime}\right)$ and hence $[\Lambda] \in \mathcal{T}(\mathbf{j})$ iff $[\Lambda] \in$ $\mathcal{T}(j) \cap \mathcal{T}\left(j^{\prime}\right)$. Therefore the only problematical vertices are those which lie on the boundary of both $\mathcal{T}(j)$ and $\mathcal{T}\left(j^{\prime}\right)$. Now one goes through all combinations as to whether $\mathcal{T}(j)$ resp. $\mathcal{T}\left(j^{\prime}\right)$ is a ball (around a vertex or a midpoint of an edge) resp. a tube, using the second assertion of corollary 2.8. In the analysis one uses the fact (comp. [17], Lemma 6.8) that if

$$
d\left([\Lambda], \mathcal{B}^{j}\right) \leq d\left([\Lambda], \mathcal{B}^{j^{\prime}}\right)
$$

then the unique geodesic from $[\Lambda]$ to $\mathcal{B}^{j^{\prime}}$ first runs along the geodesic from $[\Lambda]$ to $\mathcal{B}^{j}$ and then stays inside $\mathcal{B}^{j}$. The analysis of the extreme cases in which both $Z(j) \times_{\text {Spf } W}$ Spec $k$ and $Z\left(j^{\prime}\right) \times_{\text {Spf } W}$ Spec $k$ are sets of isolated points is left to the reader (use Proposition 2.9).

Corollary 2.13. Assume that $\mathbf{j}$ is non-degenerate of rank 2. Then $Z(\mathbf{j}) \times_{\mathrm{Spf} W}$ Spec $k$ is a projective scheme over Spec $k$. Furthermore, it is either empty, or a finite set of points, or a connected finite union of projective lines.

Proof. The first statement is equivalent to the assertion that $Z(\mathbf{j}) \times_{\operatorname{Spf} W} \operatorname{Spec} k$ is quasi-compact, i.e. to the assertion that $\mathcal{T}(\mathbf{j})$ is finite. The finiteness of $\mathcal{T}(\mathbf{j})$ follows from Proposition 2.11.

The proposition also implies that, except in extreme cases, $Z(\mathbf{j}) \times_{\operatorname{Spf} W} \operatorname{Spec} k$ is a connected union of projective lines. The extreme cases are, case by case:

(i) $\alpha=\beta=0$. Then $Z(\mathbf{j})=\emptyset$, cf. Corollary 2.12 . 
(ii) $\alpha=0, \beta>0$ (or $\beta=0, \alpha>0$ ). In this case $Z(\mathbf{j}) \times_{\operatorname{Spf} W}$ Spec $k$ consists of two ordinary special points if $\chi\left(\varepsilon_{1}\right)=-1$, or of a finite set of superspecial points if $\chi\left(\varepsilon_{1}\right)=1$. The case where $\beta=0$ and $\alpha>0$ is symmetric.

\section{$\S 3$. Local equations for the special cycles.}

In this section we will write down equations which describe a special cycle $Z(j)$ in a neighbourhood of a point $x$ in the special fibre. As mentioned in the introduction, the basic idea of how to do this is due to Genestier [7]. In the beginning of this section we fix a special endomorphism $j$ with $q(j)=\varepsilon \cdot q^{\alpha} \in \mathbb{Z}_{p}-\{0\}$.

Theorem 3.1. (Genestier) The Drinfeld isomorphism (1.2) induces isomorphisms

$$
\begin{aligned}
Z(j) \simeq \hat{\Omega}^{j} \times_{\operatorname{Spf} \mathbb{Z}_{p}} \operatorname{Spf} W & \text { if } \alpha=0 \\
Z(j) \simeq \hat{\Omega}^{\mathrm{id}+j} \times_{\operatorname{Spf} \mathbb{Z}_{p}} \operatorname{Spf} W & \text { if } \alpha>0
\end{aligned}
$$

(fixed point formal schemes for the action of elements of $G L_{2}\left(\mathbb{Q}_{p}\right)$ on $\hat{\Omega}$ ).

Proof. Suppose first that $j$ is an arbitrary element of $G\left(\mathbb{Q}_{p}\right)^{0}$ (cf. (1.1)) and define a closed formal subscheme $Z(j)$ in the same way as for a special endomorphism, cf. Definition 2.1. If $(X, \varrho) \in Z(j)(S)$, then the unique isogeny $j_{X}$ lifting $\varrho \circ j \circ \varrho^{-1}$ is of height 0 , and hence an automorphism of $X$. It follows that $(X, \varrho)$ is a fixed point for the action of $j^{-1}$ on $\mathcal{M}$. Conversely, a fixed point defines an element of $Z(j)(S)$. Therefore $Z(j)=\hat{\Omega}^{j} \times_{\operatorname{Spf} \mathbb{Z}_{p}} \operatorname{Spf} W$, since the Drinfeld isomorphism is equivariant for the action of $G\left(\mathbb{Q}_{p}\right)^{0}$, cf. (1.2).

Suppose now that $j$ is a special endomorphism with ord $\operatorname{det} j>0$. But then $\operatorname{det}(\mathrm{id}+j)=1+\operatorname{det}(j)$, hence id $+j \in G\left(\mathbb{Q}_{p}\right)^{0}$. The result therefore follows from the previous case since $Z(\mathrm{id}+j)=Z(j)$.

Our next task will be to write down equations describing the fixed point schemes. Put $\tilde{j}=j$ if $\alpha=0$ and $\tilde{j}=\mathrm{id}+j$ if $\alpha>0$. Let $x \in \hat{\Omega}^{\tilde{j}}(k)$. We distinguish two cases.

Case $x$ ordinary: After replacing $x$ by $g x$ and $j$ by $g j g^{-1}$ we may assume that $x$ lies in $\hat{\Omega}_{\left[\Lambda_{0}\right]}$, where $\Lambda_{0}=\left[e_{1}, e_{2}\right]$ denotes the standard lattice. Furthermore, ord $\operatorname{det} \tilde{j}=0$. But then, since $\tilde{j}$ fixes $x$ and hence $\left[\Lambda_{0}\right]$, it follows that $j \in M_{2}\left(\mathbb{Z}_{p}\right)$ and $\tilde{j} \in G L_{2}\left(\mathbb{Z}_{p}\right)$. We write

$$
j=\left(\begin{array}{ll}
a & b \\
c & d
\end{array}\right) .
$$


Then, using the description (1.8) of the action of $G L_{2}\left(\mathbb{Z}_{p}\right)$ on $\hat{\Omega}_{\left[\Lambda_{0}\right]}$ we obtain the following equation for $\hat{\Omega}_{\left[\Lambda_{0}\right]}^{\tilde{j}}$,

$$
-\tilde{b} T^{2}+(\tilde{a}-\tilde{d}) \cdot T+\tilde{c}=0
$$

where $\tilde{a}, \tilde{b}=b, \tilde{c}=c, \tilde{d}$ are the coefficients of $\tilde{j}$. The equation for $Z(j)$ therefore is, regardless of whether ord $\operatorname{det} j=0$ or $>0$,

$$
b T^{2}-2 a T-c=0
$$

Case $x$ superspecial: In this case, after replacing $x$ by $g x$ and $\tilde{j}$ by $g \tilde{j} g^{-1}$ we may assume that $x=p t_{\Delta_{0}}$, where $\Delta_{0}=\left(\left[\Lambda_{0}\right],\left[\Lambda_{1}\right]\right)$ is the standard edge $(1.9)$. In this case, $\tilde{j}$ will lie in the Iwahori subgroup (1.11). We write

$$
j=\left(\begin{array}{ll}
a & b \\
c & d
\end{array}\right)=\left(\begin{array}{cc}
a & p b_{0} \\
c & d
\end{array}\right)
$$

In this case we obtain from (1.12) the following pair of equations describing $\hat{\Omega}_{\Delta_{0}}^{\tilde{j}}$,

$$
\begin{aligned}
& T_{0}\left(\tilde{b}_{0} T_{0}-(\tilde{a}-\tilde{d})-\tilde{c} T_{1}\right)=0 \\
& T_{1}\left(\tilde{b}_{0} T_{0}-(\tilde{a}-\tilde{d})-\tilde{c} T_{1}\right)=0
\end{aligned}
$$

where $\tilde{a}, p \tilde{b}_{0}=p b_{0}, \tilde{c}=c, \tilde{d}$ are the coefficients of $\tilde{j}$. We have used the fact that $T_{0} T_{1}=p$. The equations for $Z(j)$ are, regardless of whether ord $\operatorname{det} j=0$ or $>0$,

$$
\begin{aligned}
& T_{0}\left(b_{0} T_{0}-2 a-c T_{1}\right)=0 \\
& T_{1}\left(b_{0} T_{0}-2 a-c T_{1}\right)=0 .
\end{aligned}
$$

The reader may reassure himself that the locus defined by the equations (3.8) induces on the open formal subscheme $\hat{\Omega}_{\left[\Lambda_{0}\right]}$ of $\hat{\Omega}_{\Delta_{0}}$, cf. (1.14), the locus defined by the equation (3.5).

In the following statement we denote by an upper index ord the intersection with the ordinary locus of $\mathcal{M}$ resp. $\hat{\Omega}$, i.e. the open formal subscheme formed by the complement of the superspecial points. Define

$$
\operatorname{mult}_{[\Lambda]}(j)=\max \left(m_{[\Lambda]}(j), 0\right),
$$

with $m_{[\Lambda]}(j)$ as defined in Lemma 2.7. 
Proposition 3.2. The closed formal subscheme $Z(j)^{\text {ord }}$ of $\left(\hat{\Omega} \times_{\operatorname{Spf} \mathbb{Z}_{p}} \text { Spf } W\right)^{\text {ord }}$ is a divisor. We have the following equality of divisors,

$$
Z(j)^{\text {ord }}=\sum_{[\Lambda] \in \mathcal{T}(j)} \operatorname{mult}_{[\Lambda]}(j) \cdot \mathbb{P}_{[\Lambda]}^{\text {ord }},
$$

unless $q(j)=\varepsilon \cdot p^{\alpha}$ where $\varepsilon \in \mathbb{Z}_{p}^{\times}$and with $\alpha$ even and $\chi(\varepsilon)=-1$, in which case

$$
Z(j)^{\text {ord }}=Z(j)^{\text {ord }, h}+\sum_{[\Lambda] \in \mathcal{T}(j)} \operatorname{mult}_{[\Lambda]}(j) \cdot \mathbb{P}_{[\Lambda]}^{\text {ord }}
$$

where $Z(j)^{\text {ord, } h}$ (the "horizontal divisor") is isomorphic to the disjoint union of two copies of $\operatorname{Spf} W$ and meets the special fibre in two ordinary special points of $\mathbb{P}_{[\Lambda(j)]}$. Here $[\Lambda(j)]$ denotes the unique vertex fixed by $j$.

Proof. We wish to determine $Z(j) \cap\left(\hat{\Omega}_{[\Lambda]} \times_{\operatorname{Spf} \mathbb{Z}_{p}} \operatorname{Spf} W\right)$ for a vertex $[\Lambda]$ where this intersection is non-empty. Note that this implies that $m_{[\Lambda]}(j) \geq 0$, cf. Proposition 2.1. Replacing $[\Lambda]$ by $[g \Lambda]$ and $j$ by $g j g^{-1}$ we may assume that $[\Lambda]=\left[\Lambda_{0}\right]$ is the standard lattice. Let us write

$$
j=\left(\begin{array}{cc}
a & b \\
c & -a
\end{array}\right)=p^{m} \cdot\left(\begin{array}{cc}
\bar{a} & \bar{b} \\
\bar{c} & -\bar{a}
\end{array}\right)=p^{m} \cdot \bar{j}, \quad m=m_{\left[\Lambda_{0}\right]}(j),
$$

where $\bar{a}, \bar{b}, \bar{c} \in \mathbb{Z}_{p}$ are not simultaneously divisible by $p$. The equation (3.5) for $Z(j) \cap\left(\hat{\Omega}_{\left[\Lambda_{0}\right]} \times_{\operatorname{Spf} \mathbb{Z}_{p}} \operatorname{Spf} W\right)$ may be written as

$$
p^{m} \cdot\left(\bar{b} T^{2}-2 \bar{a} T-\bar{c}\right)=0 .
$$

It follows already that $Z(j) \cap\left(\hat{\Omega}_{[\Lambda]} \times_{\operatorname{Spf} \mathbb{Z}_{p}}\right.$ Spf $\left.W\right)$ is a divisor. The second factor in the LHS of (3.13) is not divisible by $p$, i.e. is a unit after localizing at the ideal $(p)$. Since $\mathbb{P}_{\left[\Lambda_{0}\right]}^{\text {ord }}$ is defined by $p=0$ in $\hat{\Omega}_{\left[\Lambda_{0}\right]}$, the multiplicity of $\mathbb{P}_{\left[\Lambda_{0}\right]}$ in the divisor is equal to $m$, as asserted. We still have to determine the zero set $Z(j)^{\text {ord }, h} \cap\left(\hat{\Omega}_{\left[\Lambda_{0}\right]} \times_{\operatorname{Spf} \mathbb{Z}_{p}} \operatorname{Spf} W\right)$ of the second factor of (3.13). Now

$$
Z(j)^{\text {ord }, h} \cap\left(\hat{\Omega}_{\left[\Lambda_{0}\right]} \times_{\operatorname{Spf} \mathbb{Z}_{p}} \operatorname{Spf} W\right)=Z(\bar{j}) \cap\left(\hat{\Omega}_{\left[\Lambda_{0}\right]} \times_{\operatorname{Spf} \mathbb{Z}_{p}} \operatorname{Spf} W\right) .
$$

Furthermore, $\mathbb{P}_{\left[\Lambda_{0}\right]}^{\text {ord }} \cap Z(\bar{j})$ is precisely the set of lines in $\mathbb{P}_{\left[\Lambda_{0}\right]}^{\text {ord }}$ preserved by $\operatorname{red}_{\Lambda_{0}}(\bar{j})$. Since $\operatorname{red}_{\Lambda_{0}}(\bar{j}) \neq 0$, Proposition 2.2 implies that this last intersection is empty, unless $\operatorname{red}_{\Lambda}(\bar{j})$ is an invertible transformation of $\Lambda / p \Lambda$ and this intersection consists of two ordinary special points. In the latter case we have $j\left(\Lambda_{0}\right)=p^{m} \Lambda_{0}$, hence $\left[\Lambda_{0}\right]$ is a fixed vertex under $j$. It follows that $\alpha$ is even and $\chi(\varepsilon)=-1$, cf. Corollary 2.5 , hence $\left[\Lambda_{0}\right]$ is the unique vertex fixed by $j$. Furthermore, the extension

$$
\mathbb{Z}_{p}[T] / \bar{b} T^{2}-2 \bar{a} T-\bar{c}
$$

is unramified, which finishes the proof.

We next turn to the local equations of $Z(j)$ at a superspecial point. 
Proposition 3.3. Let $q(j)=\varepsilon \cdot p^{\alpha}, \varepsilon \in \mathbb{Z}_{p}^{\times}, \alpha \geq 0$

(i) If $\alpha=0$ and $\chi(\varepsilon)=-1$, then $Z(j)$ contains no superspecial points, $c f$. Corollary 2.6, (i)

(ii) If $\alpha=0$ and $\chi(\varepsilon)=1$, then $Z(j)$ is equal to the disjoint union of the reduced superspecial points corresponding to the edges in the fixed apartment $\mathcal{B}^{j}$, cf. Corollary 2.6, (ii).

(iii) If $\alpha \geq 1$, then $Z(j)$ is purely one-dimensional and contains superspecial points. Let $x=p t_{\Delta}$ be one of them, where $\Delta$ is an edge contained in $\mathcal{T}(j)$. Then, locally around $p t_{\Delta}, Z(j)$ is the union of a divisor with support in the special fibre and an embedded component at $p t_{\Delta}$, except in the case where $\alpha$ is odd and $\Delta=\Delta(j)$ is the edge containing the midpoint fixed by $j$. In the latter case, if $\alpha=1$, then $Z(j)$ is the union of $Z(j)^{h}$ and an embedded component at $p t_{\Delta(j)}$, where $Z(j)^{h}$ is a divisor isomorphic to Spf $W^{\prime}$ where $W^{\prime}$ is the ring of integers in the ramified quadratic extension of $W_{\mathbb{Q}}$. Finally, in the latter case, if $\alpha>1$, then $Z(j)$ is locally at $p t_{\Delta(j)}$ the union of a divisor $Z(j)^{h}=\operatorname{Spf} W^{\prime}$, an embedded component at $p t_{\Delta}$ and a divisor with support in the special fibre.

Proof. As before we may assume that $\Delta_{0}=\left(\left[\Lambda_{0}\right],\left[\Lambda_{1}\right]\right)$ is the standard simplex. Let us first assume that $\left[\Lambda_{0}\right]$ is strictly closer than $\left[\Lambda_{1}\right]$ to the fixed point set $\mathcal{B}^{j}$. We therefore have

$$
m:=m_{\left[\Lambda_{0}\right]}(j), m_{\left[\Lambda_{1}\right]}(j)=m-1 \geq 0 .
$$

In terms of the canonical basis $e_{1}, e_{2}$ of $\Lambda_{0}$ we may write

$$
j=\left(\begin{array}{cc}
a & b \\
c & -a
\end{array}\right)=p^{m} \cdot\left(\begin{array}{cc}
\bar{a} & \bar{b} \\
\bar{c} & -\bar{a}
\end{array}\right)
$$

where $\bar{a}, \bar{b}, \bar{c}$ are not simultaneously divisible by $p$. On the other hand, $b=p \cdot b_{0}$, cf. (3.6), and in terms of the canonical basis $p e_{1}, e_{2}$ of $\Lambda_{1}$ the matrix of $j$ is

$$
\left(\begin{array}{cc}
a & b_{0} \\
p c & -a
\end{array}\right)=p^{m-1}\left(\begin{array}{cc}
p \bar{a} & \bar{b}_{0} \\
p^{2} \bar{c} & -p \bar{a}
\end{array}\right),
$$

with $b_{0}=p^{m-1} \cdot \bar{b}_{0}$. Since $m_{\left[\Lambda_{1}\right]}(j)=m-1$ we conclude that $\bar{b}_{0}$ is a unit. Therefore the system of equations (3.8) for $Z(j) \cap\left(\hat{\Omega}_{\Delta_{0}} \times_{\operatorname{Spf} \mathbb{Z}_{p}} \operatorname{Spf} W\right)$ is given by

$$
\begin{array}{r}
p^{m-1} \cdot T_{0} \cdot\left(\bar{b}_{0} \cdot T_{0}-2 p \bar{a}-p \bar{c} \cdot T_{1}\right)=T_{0}^{m+1} \cdot T_{1}^{m-1} \cdot u=0 \\
p^{m-1} \cdot T_{1}\left(\bar{b}_{0} T_{0}-2 p \bar{a}-p \bar{c} \cdot T_{1}\right)=T_{0}^{m} \cdot T_{1}^{m} \cdot u=0
\end{array}
$$


where

$$
u=\bar{b}_{0}-2 \bar{a} \cdot T_{1}-\bar{c} \cdot T_{1}^{2}
$$

is a unit in the local ring of $p t_{\Delta_{0}}$. The above system of equations describes the same locus as

$$
T_{0}^{2} \cdot\left(T_{0} T_{1}\right)^{m-1}=\left(T_{0} T_{1}\right) \cdot\left(T_{0} T_{1}\right)^{m-1}=0
$$

In this case we therefore see that $Z(j)$ is the union of a divisor with support in the special fibre, and an embedded component at $p t_{\Delta_{0}}$.

Now consider the case when $\left[\Lambda_{0}\right]$ and $\left[\Lambda_{1}\right]$ have the same distance to the fixed point set. There are two alternatives.

First case: $\mathcal{B}^{j}$ is the midpoint of $\Delta_{0}$ (hence $\alpha$ is odd). In this case $j\left(\Lambda_{0}\right)$ is a scalar multiple of $\Lambda_{1}$ and $j\left(\Lambda_{1}\right)$ a scalar multiple of $\Lambda_{0}$,

$$
\begin{aligned}
& j\left(\Lambda_{0}\right)=p^{m} \cdot \Lambda_{1} \\
& j\left(\Lambda_{1}\right)=p^{m+1} \Lambda_{0} .
\end{aligned}
$$

In this case we conclude in terms of the equation (3.17) that

$$
p|\bar{a}, \quad p| \bar{b}, \quad \operatorname{ord} \operatorname{det}\left(\begin{array}{cc}
\bar{a} & \bar{b} \\
\bar{c} & -\bar{a}
\end{array}\right)=1 .
$$

Therefore $\bar{b}=p \cdot \bar{b}_{0}$, and $\bar{b}_{0}$ and $\bar{c}$ are both units. Hence the system of equations (3.8) is given by

$$
\begin{aligned}
& p^{m} \cdot T_{0}\left(\bar{b}_{0} T_{0}-2 \bar{a}-\bar{c} T_{1}\right)=0 \\
& p^{m} \cdot T_{1}\left(\bar{b}_{0} T_{0}-2 \bar{a}-\bar{c} T_{1}\right)=0 .
\end{aligned}
$$

Up to a unit in the local ring at $p t_{\Delta}$ the second factor is equal to $\bar{b}_{0} T_{0}-\bar{c} T_{1}$, hence $Z(j)$ is in a neighbourhood of $p t_{\Delta}$ defined by the equations

$$
T_{0}^{m+1} \cdot T_{1}^{m}\left(\bar{b}_{0} T_{0}-\bar{c} T_{1}\right)=T_{0}^{m} T_{1}^{m+1}\left(\bar{b}_{0} T_{0}-\bar{c} T_{1}\right)=0
$$

We therefore see that $Z(j)$ locally at $p t_{\Delta}$ is the union of the divisor $Z(j)^{h}$, with

$$
Z(j)^{h}=\operatorname{Spf} W\left[T_{0}, T_{1}\right]^{\wedge} /\left(\bar{b}_{0} T_{0}-\bar{c} T_{1}, T_{0} T_{1}-p\right) \simeq \operatorname{Spf} W[X] /\left(X^{2}-p\right)
$$

$(p \neq 2)$, an embedded component at $p t_{\Delta}$, and a divisor with support in the special fibre provided that $m>0$, i.e., $\alpha>1$. If $\alpha=1$, then $m=0$ and there is no divisor with support in the special fibre present. 
Second case: $\Delta_{0}$ lies in the fixed apartment $\mathcal{B}^{j}$ (hence $\alpha$ is even and $\chi(\varepsilon)=1$ ). In this case

$$
\begin{aligned}
& j\left(\Lambda_{0}\right)=p^{m} \Lambda_{0} \\
& j\left(\Lambda_{1}\right)=p^{m} \Lambda_{1} .
\end{aligned}
$$

In this case we conclude in terms of the equation (3.17) that

$$
p \mid \bar{b}, \operatorname{det}\left(\begin{array}{cc}
\bar{a} & \bar{b} \\
\bar{c} & -\bar{a}
\end{array}\right) \in \mathbb{Z}_{p}^{\times}
$$

We conclude that $\bar{a}$ is a unit. The system of equations (3.8) may be written as

$$
\begin{aligned}
& p^{m} \cdot T_{0}\left(\bar{b}_{0} T_{0}-2 \bar{a}-\bar{c} T_{1}\right)=0 \\
& p^{m} \cdot T_{1}\left(\bar{b}_{0} T_{0}-2 \bar{a}-\bar{c} T_{1}\right)=0,
\end{aligned}
$$

where $p \cdot \bar{b}_{0}=\bar{b}$. The second factor is a unit in the local ring at $p t_{\Delta_{0}}$ and therefore $Z(j)$ is locally around $p t_{\Delta_{0}}$ described by

$$
T_{0}^{m+1} \cdot T_{1}^{m}=T_{0}^{m} \cdot T_{1}^{m+1}=0 .
$$

If $m \geq 1$, i.e. $\alpha \geq 2$, then $Z(j)$ is the union of a divisor with support in the special fibre and an embedded component. If $m=\alpha=0$, then $Z(j)$ consists of the reduced origin only $\left(T_{0}=T_{1}=0\right)$.

Corollary 3.4. Suppose that $q(j)=\varepsilon \cdot p^{\alpha}$ with $\alpha$ even and $\chi(\varepsilon)=1$. Then the support of $Z(j)$ is contained in the special fibre.

Remark 3.5. Consider the special case $\alpha=0, \chi(\varepsilon)=1$ of the previous corollary, i.e. (ii) of Proposition 3.3. After correcting $j$ by a unit scalar, we may assume that $j^{2}=\mathrm{id}$. Let $(X, \iota) \in Z(j)(k)$. Then $j$ induces a $O_{B}$-stable decomposition into its \pm 1 -eigenspaces

$$
(X, \iota)=\left(Y_{1}, \iota_{1}\right) \times\left(Y_{2}, \iota_{2}\right)
$$

But then $\left(Y_{i}, \iota_{i}\right) \quad(i=1,2)$ are both isomorphic to the $p$-divisible group of a supersingular elliptic curve with its $O_{B}$-action. It is well-known that $\left(Y_{i}, \iota_{i}\right)$ has no non-trivial deformations, hence neither has $(X, \iota)$. This argument gives an alternative proof of assertion (ii) of Proposition 3.3.

Later, when we make the connection with the global situation, we will see a more convincing reason for the assertion of Corollary 3.4.

We next turn to the intersection of two special cycles $Z(j)$ and $Z\left(j^{\prime}\right)$. We use the notation of $(2.16)$, in particular the $\mathbb{Z}_{p}$-module $\mathbf{j}$ is supposed to be nondegenerate of rank 2 . We again write $Z(\mathbf{j})=Z(j) \cap Z\left(j^{\prime}\right)$. 
Proposition 3.6. $Z(\mathbf{j})$ has support in the special fibre.

Proof. We momentarily change notations and as in section 2 denote by $j, j^{\prime}$ generators of $\mathbf{j}$ which diagonalize the bilinear form, with matrix (2.18). We assume $Z(\mathbf{j}) \neq \emptyset$. We go through the table (2.26), leaving aside the $\emptyset$-entries. Thanks to Corollary 3.4 there are only two non-trivial possibilities.

Case 1: $\alpha$ and $\beta$ even, $\chi\left(\varepsilon_{1}\right)=\chi\left(\varepsilon_{2}\right)=-1$. In this case the only part of $Z(j)$ resp. $Z\left(j^{\prime}\right)$ with support not in the special fibre is the horizontal divisor $Z(j)^{\text {ord, } h}$ resp. $Z\left(j^{\prime}\right)^{\text {ord, } h}$ which meets the special fibre in two ordinary special points $x_{1}, x_{2} \in \mathbb{P}_{[\Lambda]}$ resp. $x_{1}^{\prime}, x_{2}^{\prime} \in \mathbb{P}_{[\Lambda]}$, cf. Propositions 3.2 and 3.3. Here $[\Lambda]=$ $[\Lambda(j)]=\left[\Lambda\left(j^{\prime}\right)\right]$ is the unique vertex fixed by $j$ and $j^{\prime}$. We may write

$$
j=p^{\alpha / 2} \cdot \bar{j}, \quad j^{\prime}=p^{\beta / 2} \cdot \bar{j}^{\prime}
$$

where $\operatorname{red}_{\Lambda}(\bar{j})$ and $\operatorname{red}_{\Lambda}\left(\bar{j}^{\prime}\right)$ are invertible traceless endomorphisms of $\Lambda / p \Lambda$ and whose eigen lines are $x_{1}$ and $x_{2}$ resp. $x_{1}^{\prime}$ and $x_{2}^{\prime}$, cf. proof of Proposition 3.2. Since these two endomorphisms anticommute, each one interchanges the eigen lines of the other (comp. proof of Proposition 2.9, (ii)), hence they have no common eigen line. We conclude that in this case

$$
Z(j)^{h} \cap Z\left(j^{\prime}\right)^{h}=\emptyset
$$

Case 2: $\alpha$ and $\beta$ odd. In this case $j$ and $j^{\prime}$ both fix the midpoint of an edge $\Delta$ and the only part of $Z(j)$ resp. $Z(j)$ with support not in the special fibre is the horizontal divisor $Z(j)^{h}$ resp. $Z\left(j^{\prime}\right)^{h}$ passing through $p t_{\Delta}$, cf. Proposition 3.3. We write, as in (3.12)

$$
j=p^{\frac{\alpha-1}{2}} \cdot \bar{j}, \quad \operatorname{resp} . \quad j^{\prime}=p^{\frac{\beta-1}{2}} \cdot \bar{j}^{\prime}
$$

where

$$
\bar{j}=\left(\begin{array}{cc}
\bar{a} & \bar{b} \\
\bar{c} & -\bar{a}
\end{array}\right), \quad \text { resp. } \bar{j}^{\prime}=\left(\begin{array}{cc}
\bar{a}^{\prime} & \bar{b}^{\prime} \\
\bar{c}^{\prime} & -\bar{a}^{\prime}
\end{array}\right)
$$

with $p$ dividing $\bar{a}, \bar{a}^{\prime}, \bar{b}, \bar{b}^{\prime}$ and where, if $\bar{b}=p \cdot b_{0}$ resp. $\bar{b}^{\prime}=p \cdot \bar{b}_{0}^{\prime}$, the elements $\bar{b}_{0}, \bar{b}_{0}^{\prime}, \bar{c}, \bar{c}^{\prime}$ are all units, cf. (3.21). Then $Z(j)^{h}$ resp. $Z\left(j^{\prime}\right)^{h}$ is defined inside Spf $W\left[T_{0}, T_{1}\right]^{\wedge} / T_{0} T_{1}-p$ by the linear equation

$$
\bar{b}_{0} T_{0}-\bar{c} T_{1}=0 \text { resp. } \bar{b}_{0}^{\prime} T_{0}-\bar{c}^{\prime} T_{1}=0
$$


cf. (3.24). We claim that these equations describe distinct subschemes which will establish the proposition. Suppose to the contrary that

$$
\bar{b}_{0} \bar{c}^{\prime}=\bar{b}_{0}^{\prime} \bar{c}
$$

However, $j$ and $j^{\prime}$ anticommute, i.e.

$$
\bar{a} \bar{a}^{\prime}+p \cdot \bar{b}_{0} \bar{c}^{\prime}=-\left(\bar{a} \bar{a}^{\prime}+p \cdot \bar{b}_{0}^{\prime} \bar{c}\right)
$$

Taking these identities together we therefore obtain

$$
\bar{a} \bar{a}^{\prime}+p \bar{b}_{0} \bar{c}^{\prime}=0
$$

But the first summand is divisible by $p^{2}$, whereas the second summand is only divisible by $p$ which is the desired contradiction.

We note the following consequence of the proof.

Corollary 3.7. Let $j$ and $j^{\prime}$ be generators of $\mathbf{j}$ which diagonalize the bilinear form, with matrix (2.18). For the horizontal divisors $Z(j)^{h}$ and $Z\left(j^{\prime}\right)^{h}$ we have $Z(j)^{h} \cap Z\left(j^{\prime}\right)^{h}=\emptyset$ unless $\alpha$ and $\beta$ are both odd. In the latter case $Z(j)^{h}$ and $Z\left(j^{\prime}\right)^{h}$ intersect transversally at a unique superspecial point.

Here, as in the rest of the paper, we formally set $Z(j)^{h}=\emptyset$ if $q(j)=\varepsilon \cdot p^{\alpha}$ with $\alpha$ even and $\chi(\varepsilon)=1$.

\section{$\S 4$. Intersection calculus of special cycles.}

Our next aim will be to determine the intersection numbers of special cycles. Before doing this we will have to explain briefly the kind of intersection theory we will want to use. The definitions and facts we need are essentially all well-known but we could not find a reference for them. The exposition in the literature closest to our needs is Deligne's [3].

Let $(S, \eta, s)$ be the spectrum of a discrete valuation ring and let $X$ be an $S$ scheme $f: X \rightarrow S$ which is regular and locally of finite type and flat over $S$ with all fibres of pure dimension 1. Let $Y=f^{-1}(s)$. For any coherent $\mathcal{O}_{X}$ - module $\mathcal{F}$

with support proper over $S$ and contained in $Y$ we may define its Euler-Poincaré characteristic

$$
\chi(\mathcal{F})=\lg f_{*} \mathcal{F}-\lg R^{1} f_{*} \mathcal{F} .
$$


Then $\chi$ is additive in short exact sequences, and if $\mathcal{F}$ is a skyscraper sheaf concentrated in $y \in Y$, then

$$
\chi(\mathcal{F})=\lg _{\mathcal{O}_{y}}\left(\mathcal{F}_{y}\right) \cdot[k(y): k(s)] .
$$

If $K$ is a complex of $\mathcal{O}_{X}$-modules with finitely many cohomology sheaves which are coherent and of the above type we set

$$
\chi(K)=\sum(-1)^{i} \chi\left(\mathcal{H}^{i}(K)\right) .
$$

Lemma 4.1. Let $\mathcal{F}$ be a skyscraper sheaf concentrated at $y \in Y$ and let $\mathcal{G}$ be any coherent $\mathcal{O}_{X}$-module with $\operatorname{supp} \mathcal{G}$ a proper closed subscheme of $X$ locally at $y$. Then

$$
\chi(\mathcal{F} \otimes \mathbb{L} \mathcal{G})=\chi(\mathcal{F} \otimes \mathcal{G})-\chi\left(\mathcal{T} \operatorname{or}_{1}(\mathcal{F}, \mathcal{G})\right)=0 .
$$

[Here $\mathcal{F} \otimes \otimes^{\mathbb{L}} \mathcal{G}$ denotes the derived tensor product of $\mathcal{F}$ and $\mathcal{G}$, a complex of quasicoherent $\mathcal{O}_{X}$-modules well defined in the derived category, with cohomology sheaves in degree $-i$ equal to $\mathcal{T}_{\text {or }}{ }_{i}^{\mathcal{O}_{X}}(\mathcal{F}, \mathcal{G})$. Since $X$ is supposed to be regular of dimension 2 , the Tor-terms for $i \geq 2$ vanish.]

Proof. The sheaves $\mathcal{T o r}_{i}(\mathcal{F}, \mathcal{G})$ are skyscraper sheaves concentrated in $y$, hence

$$
\chi\left(\mathcal{F} \otimes \otimes^{\mathbb{L}} \mathcal{G}\right)=\sum(-1)^{i} \lg \operatorname{Tor}_{i}^{\mathcal{O}_{y}}\left(\mathcal{F}_{y}, \mathcal{G}_{y}\right) .
$$

Now $\mathcal{G}_{y}$ has a resolution of length 2 by free $\mathcal{O}_{y}$-modules of finite rank,

$$
0 \rightarrow \mathcal{O}_{y}^{n_{1}} \rightarrow \mathcal{O}_{y}^{n_{2}} \rightarrow \mathcal{G}_{y} \rightarrow 0
$$

The hypothesis on $\mathcal{G}$ implies that $n_{1}=n_{2}$. Tensoring (4.5) with $\mathcal{F}_{y}$ we obtain

$$
\chi\left(\mathcal{F}_{y} \otimes_{\mathcal{O}_{y}}^{\mathbb{L}} \mathcal{G}_{y}\right)=n_{1} \cdot \lg \mathcal{F}_{y}-n_{2} \lg \mathcal{F}_{y}=0 .
$$

Let now $Z$ and $Z^{\prime}$ be closed subschemes of $X$ such that $\left(Z \cap Z^{\prime}\right)_{\text {red }}$ is contained in $Y$ and is proper over $S$. We then define their intersection number by

$$
\begin{aligned}
\left(Z, Z^{\prime}\right) & =\chi\left(\mathcal{O}_{Z} \otimes^{\mathbb{L}} \mathcal{O}_{Z^{\prime}}\right) \\
& =\chi\left(\mathcal{O}_{Z} \otimes \mathcal{O}_{Z^{\prime}}\right)-\chi\left(\mathcal{T o r}_{1}\left(\mathcal{O}_{Z}, \mathcal{O}_{Z^{\prime}}\right)\right) .
\end{aligned}
$$


If $Z$ and $Z^{\prime}$ are divisors which meet in a finite number of points contained in $Y$ then

$$
\left(Z, Z^{\prime}\right)=\sum_{y \in Z \cap Z^{\prime}} \lg \left(\mathcal{O}_{Z} \otimes \mathcal{O}_{Z^{\prime}}\right)_{y}
$$

is the intersection number in the most naive sense. In $[\mathbf{3}]$ Deligne defines the intersection number of two divisors by formula (4.6), if one of the two divisors is concentrated in $Y$ (he also assumes $f$ to be proper). We will see now that the general case essentially reduces to the case of divisors.

Let $Z$ be a closed subscheme of $X$ and define $Z^{\text {pure }}$ to be the closed subscheme of $Z$ defined by the ideal sheaf of local sections with finite support. In particular, if $Z$ is zero-dimensional, then $Z^{\text {pure }}=\emptyset$.

Lemma 4.2. $Z^{\text {pure }}$ is Cohen-Macauley, i.e. has no embedded components. If $\operatorname{dim} Z=1$, then $Z^{\text {pure }}$ is a divisor on $X$ (i.e. defined locally by one non-zero element).

Proof. The cases when $\operatorname{dim} Z=2$ or $\operatorname{dim} Z=0$ are trivial. Now let $\operatorname{dim} Z=1$. Then the first assertion implies the second (EGA IV, 21.7.2, 21.6.9, 21.11.1). The first assertion is local around a point $y \in Y$. If $Z^{\text {pure }}$ had an embedded prime ideal at $y$, it would have to be the maximal ideal $\mathfrak{m}_{y}$ of $\mathcal{O}_{y}$. But then there would exist $a \in \mathcal{O}_{y}$ such that

$$
\mathfrak{m}_{y}=\operatorname{rad}(I: a)
$$

([1] $]$, th. 4.5). Here $I \subset \mathcal{O}_{y}$ is the ideal of $Z^{\text {pure }}$. But then

$$
\mathfrak{m}_{y}^{n} \cdot a \subset I, \quad \text { some } n,
$$

hence $a$ would define a local section of $\mathcal{O}_{Z^{\text {pure }}}$ with support in $y$. By the definition of $Z^{\text {pure }}$ this implies $a \in I$, a contradiction.

The next statement is analogous to Theorem 2.3.8, (iii) of Deligne [3].

Lemma 4.3. Let $Z$ and $Z^{\prime}$ be proper closed subschemes of $X$ such that their intersection number (4.6) is defined. Then

$$
\left(Z, Z^{\prime}\right)=\left(Z^{\text {pure }}, Z^{\prime \text { pure }}\right)
$$


Proof. There are exact sequences

$$
\begin{aligned}
& 0 \rightarrow \mathcal{J} \rightarrow \mathcal{O}_{Z} \rightarrow \mathcal{O}_{Z^{\text {pure }}} \rightarrow 0 \\
& 0 \rightarrow \mathcal{J}^{\prime} \rightarrow \mathcal{O}_{Z^{\prime}} \rightarrow \mathcal{O}_{Z^{\prime} \text { pure }} \rightarrow 0
\end{aligned}
$$

where $\mathcal{J}$ and $\mathcal{J}^{\prime}$ have finite support contained in $Y$. Using Lemma 4.1 and the bilinearity of the tensor product and the additivity of $\chi$, the result follows.

One last fact we need is the following (Deligne [3]): Assume that the morphism $f$ is proper and let $Z$ be a closed subscheme with support in $Y$. Then

$$
\left(Z, f^{-1}(s)\right)=0
$$

Remark 4.4. The preceding theory applies equally well to the case when $X$ is a formal scheme which is regular and where $f: X \rightarrow S$ is an adic morphism into the formal spectrum of a complete discrete valuation ring which is flat and locally of finite type and with one-dimensional fibre over $s \in S$. In this case we have defined the intersection number of closed formal subschemes $Z$ and $Z^{\prime}$ such that the sum of their defining ideals is open in $\mathcal{O}_{X}$, with the same properties as before. Furthermore, there is an obvious compatibility between these notions: if the adic morphism and the closed formal subschemes are formal completions along the special fibre of a morphism of schemes and of closed subschemes then both intersection numbers coincide.

We now return to the case of interest, namely to the formal scheme $\mathcal{M} \simeq$ $\hat{\Omega} \times{ }_{\text {Spf } \mathbb{Z}_{p}} \operatorname{Spf} W$ over Spf $W$. By Lemma 4.3 we need to know the divisors $Z(j)^{\text {pure }}$. The following proposition is an immediate consequence of Propositions 3.2 and 3.3.

Proposition 4.5. Let $q(j)=\varepsilon \cdot p^{\alpha}, \varepsilon \in \mathbb{Z}_{p}^{\times}, \alpha \geq 0$.

(i) If $\alpha$ is even and $\chi(\varepsilon)=1$, then

$$
Z(j)^{\text {pure }}=\sum_{[\Lambda]} \operatorname{mult}_{[\Lambda]}(j) \cdot \mathbb{P}_{[\Lambda]}
$$

(equality of divisors on $\mathcal{M}$ ).

(ii) If $\alpha$ is even and $\chi(\varepsilon)=-1$, then

$$
Z(j)^{\text {pure }}=Z(j)^{h}+\sum_{[\Lambda]} \operatorname{mult}_{[\Lambda]}(j) \cdot \mathbb{P}_{[\Lambda]}
$$


where $Z(j)^{h}$ (the horizontal part of $\left.Z(j)\right)$ is the disjoint sum of two divisors each projecting isomorphically to $\operatorname{Spf} W$ and meeting the special fibre in an ordinary special point of $\mathbb{P}_{[\Lambda(j)]}$. Here $[\Lambda(j)]$ denotes the unique vertex fixed by $j$.

(iii) If $\alpha$ is odd, then

$$
Z(j)^{\text {pure }}=Z(j)^{h}+\sum_{[\Lambda]} \operatorname{mult}_{[\Lambda]}(j) \mathbb{P}_{[\Lambda]}
$$

where the divisor $Z(j)^{h}$ is the formal spectrum of the ring of integers in a ramified quadratic extension of $W_{\mathbb{Q}}$ which meets the special fibre in $p t_{\Delta(j)}$, where $\Delta(j)$ is the edge containing the unique fixed point of $j$.

In particular, in all cases the divisor $Z(j)^{\text {pure }}$ is the sum with multiplicities of regular one-dimensional formal schemes (or empty).

Remark 4.6. Genestier [7] has formulated a moduli problem (over $\mathcal{M}$ ) whose solution is $Z(j)$ pure . The reason that we stick with the "uglier" subscheme $Z(j)$ is that its definition can be transposed easily to other cases, $[\mathbf{1 6}],[\mathbf{1 7}]$. Due to Lemma 4.3 the difference between $Z(j)$ and $Z(j)^{\text {pure }}$ has no consequences for the intersection numbers.

Next we will have to determine the intersection numbers between all the various summands of $Z(j)^{\text {pure }}$ resp. $Z\left(j^{\prime}\right)^{\text {pure }}$.

Lemma 4.7. For any pair of vertices $[\Lambda],\left[\Lambda^{\prime}\right]$ we have

$$
\left(\mathbb{P}_{[\Lambda]}, \mathbb{P}_{\left[\Lambda^{\prime}\right]}\right)= \begin{cases}1 & \text { if }\left([\Lambda],\left[\Lambda^{\prime}\right]\right) \text { is an edge } \\ -(p+1) & \text { if }[\Lambda]=\left[\Lambda^{\prime}\right] \\ 0 & \text { in all other cases. }\end{cases}
$$

Proof. The first and the last entry on the RHS are obvious. Let $\Gamma \subset P G L_{2}\left(\mathbb{Q}_{p}\right)$ be a cocompact discrete subgroup. Then $\hat{\Omega} / \Gamma$ is a formal scheme which is proper and flat over $\operatorname{Spf} \mathbb{Z}_{p}$. If $\Gamma$ is sufficiently small the projection

$$
\hat{\Omega} \rightarrow \hat{\Omega} / \Gamma
$$

is locally around $\mathbb{P}_{[\Lambda]}$ an isomorphism. The special fibre $Y$ of $\hat{\Omega} / \Gamma$ is reduced and is of the form

$$
Y=\sum_{[\Lambda] \bmod \Gamma} \mathbb{P}_{[\Lambda]}
$$


Using now (4.9) we obtain

$$
\begin{gathered}
0=\left(\mathbb{P}_{[\Lambda]}, Y\right)=\left(\mathbb{P}_{[\Lambda]},\left(\sum_{\left[\Lambda^{\prime}\right]} \mathbb{P}_{\left[\Lambda^{\prime}\right]}\right)\right) \\
=\left(\mathbb{P}_{[\Lambda]}, \mathbb{P}_{[\Lambda]}\right)+\sum_{\substack{\left[\Lambda^{\prime}\right] \\
\left[\Lambda^{\prime}\right] \neq[\Lambda]}}\left(\mathbb{P}_{[\Lambda]}, \mathbb{P}_{\left[\Lambda^{\prime}\right]}\right) .
\end{gathered}
$$

But $\mathbb{P}_{[\Lambda]}$ meets precisely $p+1$ other irreducible components and with multiplicity one. The result follows.

Lemma 4.8. Let $\mathbf{j}$ be a non-degenerate $\mathbb{Z}_{p}$-submodule of rank 2 and let $j, j^{\prime}$ be generators of $\mathbf{j}$ which diagonalize the bilinear form, with matrix (2.18). For the horizontal part of the associated divisors $Z(j)^{\text {pure }}$ resp. $Z\left(j^{\prime}\right)^{\text {pure }}$ we have

$$
\left(Z(j)^{h}, Z\left(j^{\prime}\right)^{h}\right)= \begin{cases}1 & \text { if } \alpha \text { and } \beta \text { odd } \\ 0 & \text { otherwise }\end{cases}
$$

Proof. This follows from Corollary 3.7.

Lemma 4.9. Let $q(j)=\varepsilon \cdot p^{\alpha}$ and let $[\Lambda]$ be a vertex. Then

$$
\left(Z(j)^{h}, \mathbb{P}_{[\Lambda]}\right)= \begin{cases}2 & \text { if } \alpha \text { is even and } \chi(\varepsilon)=-1 \text { and } \mathcal{B}^{j}=\{[\Lambda]\} \\ 1 & \text { if } \alpha \text { is odd and } d\left([\Lambda], \mathcal{B}^{j}\right)=\frac{1}{2} \\ 0 & \text { in all other cases. }\end{cases}
$$

Proof. This follows immediately from Propositions 3.2 and 3.3 and the local equations for $Z(j)^{h}$ appearing in their proofs ((3.15) resp. (3.24)).

\section{$\S 5$. An invariance property of intersection numbers.}

The aim of this section is to establish the following invariance property of the intersection numbers of special cycles.

Theorem 5.1. Let $\left(j_{1}, j_{2}\right)$ resp. $\left(j_{1}^{\prime}, j_{2}^{\prime}\right)$ be two $\mathbb{Z}_{p}$-bases of the same non-degenerate submodule $\mathbf{j}$ of $V$ with $q\left(j_{i}\right) \in \mathbb{Z}_{p} \backslash\{0\}$ and $q\left(j_{i}^{\prime}\right) \in \mathbb{Z}_{p} \backslash\{0\}, i=1,2$. Then

$$
\mathcal{O}_{Z\left(j_{1}\right)} \otimes^{\mathbb{L}} \mathcal{O}_{Z\left(j_{2}\right)} \simeq \mathcal{O}_{Z\left(j_{1}^{\prime}\right)} \otimes^{\mathbb{L}} \mathcal{O}_{Z\left(j_{2}^{\prime}\right)}
$$


and hence

$$
\left(Z\left(j_{1}\right), Z\left(j_{2}\right)\right)=\left(Z\left(j_{1}^{\prime}\right), Z\left(j_{2}^{\prime}\right)\right) .
$$

In the statement of the theorem it is obvious that the zero'th cohomology sheaves of these complexes are isomorphic since both of them are equal to the structure sheaf of $Z(\mathbf{j})$. The statement is also obvious locally around an isolated ordinary point of $Z(\mathbf{j})$ since here the special cycles are divisors and hence there are no higher Torterms if the intersection is zero-dimensional. But the full statement is non-trivial. One basic ingredient of its proof will be to show that the Genestier equations (3.5) and (3.8) globalize to give a resolution of the structure sheaf of a special cycle.

We therefore start with a single special cycle $Z(j)$. Let us suppose that the matrix of $j$ in terms of the standard basis $e_{1}, e_{2}$ is

$$
j=\left(\begin{array}{cc}
a & b \\
c & -a
\end{array}\right) .
$$

If $p t_{\Delta_{0}} \in Z(j)$, then $p \mid b$ and writing $b=p b_{0}$ we have the global section of $\mathcal{O}$ on $\hat{\Omega}_{\Delta_{0}}$,

$$
f=b_{0} T_{0}-2 a-c T_{1} .
$$

Recall from (3.8) that the Genestier equations for the intersection $\hat{\Omega}_{\Delta_{0}} \cap Z(j)$ are given as

$$
f \cdot T_{0}=f \cdot T_{1}=0 .
$$

This leads us to consider the complex of free $\mathcal{O}$-modules on $\hat{\Omega}_{\Delta_{0}}$ concentrated in degrees $-2,-1,0$,

$$
K(1, j): \mathcal{O} \stackrel{\left(\begin{array}{c}
-T_{1} \\
T_{0}
\end{array}\right)}{\longrightarrow} \oplus \mathcal{O} \stackrel{\left(f \cdot T_{0}, f \cdot T_{1}\right)}{\longrightarrow} \mathcal{O} .
$$

We specify here that we consider an element in degree -1 as a column vector.

By what we said above $K(1, j)$ is a free resolution of the structure sheaf of $\hat{\Omega}_{\Delta_{0}} \cap Z(j)$. In order to calculate with this complex, we write

$$
f=f(j)=\operatorname{tr}\left(j \cdot \tau \cdot \operatorname{diag}\left(-p^{-1}, 1\right)\right)
$$

with

$$
\tau=\left(\begin{array}{cc}
p & -T_{1} \\
-T_{0} & 1
\end{array}\right)=\left(\begin{array}{cc}
T_{0} T_{1} & -T_{1} \\
-T_{0} & 1
\end{array}\right)
$$


From now on we have no further use of the matrix representation of $j$ and the symbols $a, b, c$ will denote the entries of other matrices appearing in the proofs below.

More generally, let $g \in G\left(\mathbb{Q}_{p}\right)^{0}$ such that $g \cdot p t_{\Delta_{0}} \in Z(j)$. We have the homomorphism of sheaves (covering the automorphism $g$ )

$$
g_{*}: \mathcal{O}_{\hat{\Omega}} \longrightarrow \mathcal{O}_{\hat{\Omega}}
$$

The global sections $g_{*}\left(T_{0}\right), g_{*}\left(T_{1}\right)$ of $\mathcal{O}_{\hat{\Omega}_{g \Delta_{0}}}$ are just the global sections constructed like $T_{0}, T_{1}$ but starting from the basis $\left[g e_{1}, g e_{2}\right]$ instead of $\left[e_{1}, e_{2}\right]$. We then obtain a complex $K(g, j)$ of $\mathcal{O}$-modules on $\hat{\Omega}_{g \Delta_{0}}$ by using in (5.2) instead of $T_{0}, T_{1}$ the global coordinates on $\hat{\Omega}_{g \Delta_{0}}$ obtained from $T_{0}, T_{1}$ by transport via $g_{*}$ and in (5.1) the coefficients of the matrix of $j$ in terms of the basis $g e_{1}, g e_{2}$. We have an obvious isomorphism (the identity)

$$
g^{*}\left(K\left(g, g j g^{-1}\right)\right) \longrightarrow K(1, j)
$$

which we prefer to view as a $g_{*}$-linear homomorphism (between sheaves on different spaces

$$
g_{*}: K(1, j) \longrightarrow K\left(g, g j g^{-1}\right),
$$

i.e. $g_{*}(x s)=g_{*}(x) \cdot g_{*}(s)$ for a section $x$ of $\mathcal{O}$ and a section $s$ of $K(1, j)$.

Lemma 5.2. Assume $p t_{\Delta_{0}} \in Z(j)$. For every element $g$ in the Iwahori subgroup (1.11) associated to $\Delta_{0}$ there is a $g_{*}^{-1}$-linear isomorphism of complexes of $\mathcal{O}$ modules on $\hat{\Omega}_{\Delta_{0}}$

$$
\lambda_{g}^{0}: K(1, j) \longrightarrow K\left(1, g^{-1} j g\right)
$$

These isomorphisms satisfy the transitivity condition

$$
\lambda_{g_{1} g_{2}}^{0}=\lambda_{g_{2}}^{0} \circ \lambda_{g_{1}}^{0}
$$

Proof. The isomorphism will be given in the following form

$$
\begin{array}{ccccccc}
K(1, j) & : & \mathcal{O} & \stackrel{\left(\begin{array}{c}
-T_{1} \\
T_{0}
\end{array}\right)}{\longrightarrow} \mathcal{O} \oplus \mathcal{O} & \stackrel{\left(f T_{0}, f T_{1}\right)}{\longrightarrow} & \mathcal{O} \\
\lambda_{g}^{0} \downarrow & \alpha \cdot \downarrow & \beta \cdot \downarrow & 1 \cdot \downarrow \\
K\left(1, g^{-1} j g\right) & : & \mathcal{O} & \stackrel{\left(\begin{array}{c}
-T_{1} \\
T_{0}
\end{array}\right)}{\longrightarrow} \mathcal{O} \oplus \mathcal{O} & \stackrel{\left(f^{\prime} T_{0}, f^{\prime} T_{1}\right)}{\longrightarrow} & \mathcal{O},
\end{array}
$$


where

$$
\alpha \in \mathcal{O}^{\times} \quad, \quad \beta=\operatorname{diag}\left(\beta_{0}, \beta_{1}\right) \in G L_{2}(\mathcal{O})
$$

Of course, we are using here the usual convention for describing a semi-linear map by a matrix, e.g. in degree -2 a section $x$ of $\mathcal{O}$ is mapped to $\alpha \cdot g_{*}^{-1}(x)$. Also we have abbreviated $f\left(g^{-1} j g\right)$ into $f^{\prime}$.

Let us calculate $f^{\prime}$. We have

$$
\begin{aligned}
f^{\prime} & =\operatorname{tr}\left(g^{-1} j g \cdot \tau \cdot \operatorname{diag}\left(-p^{-1}, 1\right)\right) \\
& =\operatorname{tr}\left(j \cdot \tau^{\prime} \cdot \operatorname{diag}\left(-p^{-1}, 1\right)\right),
\end{aligned}
$$

with

$$
\tau^{\prime}=g \cdot \tau \cdot \operatorname{diag}\left(-p^{-1}, 1\right) \cdot g^{-1} \cdot \operatorname{diag}\left(-p^{-1}, 1\right)^{-1}
$$

Now

$$
\tau=\left(\begin{array}{c}
T_{1} \\
-1
\end{array}\right) \cdot\left(T_{0},-1\right)
$$

Let

$$
g=\left(\begin{array}{ll}
a & b \\
c & d
\end{array}\right)=\left(\begin{array}{cc}
a & p b_{0} \\
c & d
\end{array}\right)
$$

Then

$$
g \cdot\left(\begin{array}{c}
T_{1} \\
-1
\end{array}\right)=\left(\begin{array}{cc}
a T_{1} & -b \\
c T_{1} & -d
\end{array}\right)=\left(-c T_{1}+d\right) \cdot\left(\begin{array}{c}
g_{*}^{-1}\left(T_{1}\right) \\
-1
\end{array}\right)
$$

where we used the expression (1.12) for $g_{*}^{-1}\left(T_{1}\right)$. We note that

$$
v=v\left(g, T_{1}\right)=-c T_{1}+d
$$

is an automorphy factor for the action of the Iwahori subgroup on $\mathcal{O}^{\times}$, i.e.

$$
v\left(g g^{\prime}, T_{1}\right)=v\left(g, g_{*}^{\prime-1}\left(T_{1}\right)\right) \cdot v\left(g^{\prime}, T_{1}\right)
$$

Similarly we have

$$
\begin{aligned}
\left(T_{0},-1\right) \cdot \operatorname{diag}\left(-p^{-1}, 1\right) \cdot g^{-1} \cdot \operatorname{diag}\left(-p^{-1}, 1\right)^{-1} \\
\quad=\left(T_{0},-1\right) \cdot \operatorname{det}(g)^{-1} \cdot\left(\begin{array}{cc}
d & b_{0} \\
p c & a
\end{array}\right) \\
\quad=\operatorname{det}(g)^{-1} \cdot\left(d T_{0}-p c, b_{0} T_{0}-a\right) \\
\quad=\operatorname{det}(g)^{-1} \cdot\left(-b_{0} T_{0}+a\right) \cdot\left(g_{*}^{-1}\left(T_{0}\right),-1\right) .
\end{aligned}
$$


Again

$$
u=u\left(g, T_{0}\right)=-b_{0} T_{0}+a
$$

is an automorphy factor valued in $\mathcal{O}^{\times}$. Plugging in these expressions into (5.8) we therefore obtain

$$
\tau^{\prime}=\operatorname{det}(g)^{-1} u v \cdot g_{*}^{-1}(\tau)
$$

and

$$
f^{\prime}=\operatorname{det}(g)^{-1} u v \cdot g_{*}^{-1}(f)
$$

We also note that

$$
\frac{g_{*}^{-1}\left(T_{0}\right)}{T_{0}}=\frac{-c T_{1}+d}{-b_{0} T_{0}+a}=\frac{v}{u}=\left(\frac{g_{*}^{-1}\left(T_{1}\right)}{T_{1}}\right)^{-1} .
$$

We now fill in the diagram (5.6) by setting

$$
\alpha=\operatorname{det}(g) \cdot u^{-1} v^{-1}, \beta_{0}=\operatorname{det}(g) \cdot u^{-2}, \beta_{1}=\operatorname{det}(g) \cdot v^{-2} .
$$

We claim that the diagram commutes.

Left square: Via the NE passage the element 1 is mapped to

$$
1 \longmapsto\left(\begin{array}{c}
-T_{1} \\
T_{0}
\end{array}\right) \longmapsto\left(\begin{array}{c}
-\beta_{0} \cdot g_{*}^{-1}\left(T_{1}\right) \\
\beta_{1} \cdot g_{*}^{-1}\left(T_{0}\right)
\end{array}\right)
$$

Via the SW passage the element 1 is mapped to

$$
1 \longmapsto \alpha \longmapsto \alpha \cdot\left(\begin{array}{c}
-T_{1} \\
T_{0}
\end{array}\right)
$$

The claim follows from the definitions (5.17) and the identity (5.16).

Right square: The element $\left(\begin{array}{l}1 \\ 0\end{array}\right)$ is mapped via the NE passage to

$$
\left(\begin{array}{l}
1 \\
0
\end{array}\right) \longmapsto f \cdot T_{0} \longmapsto g_{*}^{-1}(f) \cdot g_{*}^{-1}\left(T_{0}\right)
$$

and via the SW passage to

$$
\left(\begin{array}{l}
1 \\
0
\end{array}\right) \longmapsto \beta_{0} \cdot\left(\begin{array}{l}
1 \\
0
\end{array}\right) \longmapsto \beta_{0} \cdot f^{\prime} \cdot T_{0} .
$$


The last element is equal to by (5.15)

$$
\beta_{0} \cdot \operatorname{det}(g)^{-1} u v \cdot g_{*}^{-1}(f) \cdot T_{0}=\frac{v}{u} \cdot T_{0} \cdot g_{*}^{-1}(f)
$$

Again the relation (5.16) gives the claim. The element $\left(\begin{array}{l}0 \\ 1\end{array}\right)$ is treated in a similar way. We therefore have constructed the homomorphism $\lambda_{g}^{0}$ and it is immediate to check that $\lambda_{g}^{0}$ is in fact an isomorphism. The transitivity assertion is an immediate consequence of the fact that $u$ and $v$ are automorphy factors.

We now use the system of isomorphisms constructed above to construct a welldefined complex on each open chart $\hat{\Omega}_{\Delta}$ of $\hat{\Omega}$. If $p t_{\Delta} \notin Z(j)$ this complex is zero by definition. Next suppose that $p t_{\Delta} \in Z(j)$ and let $g_{1}, g_{2} \in G\left(\mathbb{Q}_{p}\right)^{0}$ such that $g_{1} \Delta_{0}=g_{2} \Delta_{0}=\Delta$. Then $g_{2}=g_{1} \cdot h$ where $h$ lies in the Iwahori subgroup for $\Delta_{0}$. We define a (linear) isomorphism of complexes on $\hat{\Omega}_{\Delta}$

$$
\lambda_{g_{2}, g_{1}}: K\left(g_{1}, j\right) \longrightarrow K\left(g_{2}, j\right)
$$

by putting

$$
\lambda_{g_{2}, g_{1}}=g_{2 *} \circ \lambda_{h}^{0} \circ g_{1 *}^{-1}
$$

The transitivity assertion of Lemma (5.2) yields

$$
\lambda_{g_{3}, g_{2}} \circ \lambda_{g_{2}, g_{1}}=\lambda_{g_{3}, g_{1}}
$$

for any $g_{3}$ with $g_{3} \Delta_{0}=\Delta$. This is the precise meaning of the well-definedness of the complex on $\hat{\Omega}_{\Delta}$.

Our next aim is to glue the complexes just constructed on common overlaps of local charts.

Lemma 5.3. Assume $p t_{\Delta_{0}} \in Z(j)$. For any $g \in G L_{2}\left(\mathbb{Z}_{p}\right)$ there is a $g_{*}^{-1}$-linear isomorphism of complexes of $\mathcal{O}$-modules on $\hat{\Omega}_{\left[\Lambda_{0}\right]}$,

$$
\mu_{g}^{0}: K(1, j)_{\mid \hat{\Omega}_{\left[\Lambda_{0}\right]}} \stackrel{\sim}{\longrightarrow} K\left(1, g^{-1} j g\right)_{\mid \hat{\Omega}_{\left[\Lambda_{0}\right]}}
$$

These isomorphisms satisfy the transitivity condition

$$
\mu_{g_{1} g_{2}}^{0}=\mu_{g_{2}}^{0} \circ \mu_{g_{1}}^{0}
$$


Furthermore, if $g$ lies in the Iwahori subgroup corresponding to $\Delta_{0}$, then $\mu_{g}^{0}=$ $\lambda_{g \mid \hat{\Omega}_{\left[\Lambda_{0}\right]}^{0}}^{0}$.

Proof. Taking into account the definition (1.15) of the open immersion, the complexes in question have the following form in terms of the natural coordinate $T$ on $\hat{\Omega}_{\left[\Lambda_{0}\right]}$.

$$
\begin{array}{cccccc}
K(1, j)_{\mid \hat{\Omega}_{\left[\Lambda_{0}\right]}}: & \mathcal{O} & \stackrel{\left(\begin{array}{c}
-T^{-1} \\
p T
\end{array}\right)}{\longrightarrow} \mathcal{O} \oplus \mathcal{O} & \stackrel{\left(f \cdot p T, f \cdot T^{-1}\right)}{\longrightarrow} & \mathcal{O} \\
\mu_{g}^{0} \downarrow & \alpha \cdot \downarrow & \beta \cdot \downarrow & 1 \cdot \downarrow \\
K\left(1, g^{-1} j g\right)_{\mid \hat{\Omega}_{\left[\Lambda_{0}\right]}}: & \mathcal{O} & \stackrel{\left(\begin{array}{c}
-T^{-1} \\
\stackrel{p T}{\longrightarrow}
\end{array}\right)}{\longrightarrow} & & & \\
& & & \stackrel{\left(f^{\prime} \cdot p T, f^{\prime} \cdot T^{-1}\right)}{\longrightarrow} & \mathcal{O}
\end{array}
$$

Here $f$ and $f^{\prime}$ are the restrictions of the functions appearing in (5.6) and our aim is to define $\alpha \in \mathcal{O}^{\times}$and $\beta=\operatorname{diag}\left(\beta_{0}, \beta_{1}\right) \in G L_{2}(\mathcal{O})$ to produce a commutative diagram. Let us calculate $f$ and $f^{\prime}$. We have

$$
\begin{gathered}
f=\operatorname{tr}\left(j \cdot\left(\begin{array}{cc}
p & -T^{-1} \\
-p T & 1
\end{array}\right) \cdot \operatorname{diag}\left(-p^{-1}, 1\right)\right), \text { i.e. } \\
f=\operatorname{tr}\left(j \cdot\left(\begin{array}{cc}
-1 & -T^{-1} \\
T & 1
\end{array}\right)\right) .
\end{gathered}
$$

Hence

$$
f^{\prime}=\operatorname{tr}\left(j \cdot g \cdot\left(\begin{array}{cc}
-1 & -T^{-1} \\
T & 1
\end{array}\right) g^{-1}\right)
$$

We write

$$
\left(\begin{array}{cc}
-1 & -T^{-1} \\
T & 1
\end{array}\right)=\left(\begin{array}{c}
-1 \\
T
\end{array}\right)\left(1, T^{-1}\right)
$$

Let

$$
g=\left(\begin{array}{ll}
a & b \\
c & d
\end{array}\right) \in G L_{2}\left(\mathbb{Z}_{p}\right)
$$

Then

$$
g \cdot\left(\begin{array}{c}
-1 \\
T
\end{array}\right)=\left(\begin{array}{cc}
b T & -a \\
d T & -c
\end{array}\right)=(-b T+a)\left(\begin{array}{c}
-1 \\
g_{*}^{-1}(T)
\end{array}\right),
$$

where we use (1.8). We again note that

$$
u=-b T+a
$$


is an automorphy factor for the action of $G L_{2}\left(\mathbb{Z}_{p}\right)$ on $\mathcal{O}^{\times}$. Similarly we have

$$
\begin{aligned}
\left(1, T^{-1}\right) \cdot g^{-1} & =\operatorname{det}(g)^{-1} \cdot\left(d-c T^{-1}, b+a T^{-1}\right) \\
& =\operatorname{det}(g)^{-1} \cdot\left(d-c T^{-1}\right) \cdot\left(1, g_{*}^{-1}(T)^{-1}\right)
\end{aligned}
$$

Again

$$
v=d-c T^{-1}
$$

is an automorphy factor valued in $\mathcal{O}^{\times}$. We thus obtain

$$
f^{\prime}=\operatorname{det}(g)^{-1} \cdot u v \cdot g_{*}^{-1}(f)
$$

We also note that

$$
\frac{g_{*}^{-1}(T)}{T}=\frac{v}{u}
$$

We now fill in diagram (5.21) by setting

$$
\alpha=\operatorname{det}(g) \cdot u^{-1} v^{-1}, \beta_{0}=\operatorname{det}(g) \cdot u^{-2}, \beta_{1}=\operatorname{det}(g) \cdot v^{-2} .
$$

The commutativity of the diagram is checked as before. The transitivity property again follows from the fact that $u$ and $v$ are automorphy factors. Finally, if $g$ lies in the Iwahori subgroup the matrix entries $\alpha, \beta_{0}, \beta_{1}$ of $\mu_{g}^{0}$ are just the restrictions of the corresponding entries of $\lambda_{g}^{0}$ since

$$
\begin{aligned}
& u\left(g, T_{0}\right)_{\mid \hat{\Omega}_{\left[\Lambda_{0}\right]}}=\left(-b_{0} T_{0}+a\right)_{\mid \hat{\Omega}_{\left[\Lambda_{0}\right]}}=-b T+a=u \\
& v\left(g, T_{1}\right)_{\mid \hat{\Omega}_{\left[\Lambda_{0}\right]}}=\left(-c T_{1}+d\right)_{\mid \hat{\Omega}_{\left[\Lambda_{0}\right]}}=d-c T^{-1}=v .
\end{aligned}
$$

Lemma 5.4. Assume $p t_{\Delta_{0}} \in Z(j)$. Let

$$
w=\left(\begin{array}{ll}
0 & p \\
1 & 0
\end{array}\right)
$$

so that $w G L_{2}\left(\mathbb{Z}_{p}\right) w^{-1}$ is the stabilizer of the lattice $\Lambda_{1}=\left[p e_{1}, e_{2}\right]$. For any $g \in w G L_{2}\left(\mathbb{Z}_{p}\right) w^{-1}$ there is a $g_{*}^{-1}$-linear isomorphism of complexes of $\mathcal{O}$-modules on $\hat{\Omega}_{\left[\Lambda_{1}\right]}$,

$$
\mu_{g}^{0}: K(1, j)_{\mid \hat{\Omega}_{\left[\Lambda_{1}\right]}} \stackrel{\sim}{\longrightarrow} K\left(1, g^{-1} j g\right)_{\mid \hat{\Omega}_{\left[\Lambda_{1}\right]}}
$$


These isomorphisms satisfy the transitivity condition and if $g$ lies in the Iwahori subgroup corresponding to $\Delta_{0}$ we have $\mu_{g}^{0}=\lambda_{g \mid \hat{\Omega}_{\left[\Lambda_{1}\right]}^{0}}^{0}$.

Proof. The element $w$ lies in the normalizer of the Iwahori subgroup and hence acts on $\hat{\Omega}_{\Delta_{0}}$. The action is given formally by the same formula as in (1.12), hence interchanges $T_{0}$ and $T_{1}$. Furthermore the action takes the open immersion of $\hat{\Omega}_{\left[\Lambda_{1}\right]}$ in $\hat{\Omega}_{\Delta_{0}}$ into the open immersion of $\hat{\Omega}_{\left[\Lambda_{0}\right]}$ in $\hat{\Omega}_{\Delta_{0}}$. Hence after identifying $\hat{\Omega}_{\left[\Lambda_{1}\right]}$ with $\hat{\Omega}_{\left[\Lambda_{0}\right]}$ via $w$, this open immersion is given by

$$
T_{0} \longmapsto T^{-1} \quad, \quad T_{1} \longmapsto p T
$$

For the restriction of $f$ we therefore obtain

$$
f=\operatorname{tr}\left(j \cdot\left(\begin{array}{cc}
p & -p T \\
-T^{-1} & 1
\end{array}\right) \cdot \operatorname{diag}\left(-p^{-1}, 1\right)\right)
$$

We write

$$
\left(\begin{array}{cc}
p & -p T \\
-T^{-1} & 1
\end{array}\right) \cdot \operatorname{diag}\left(-p^{-1}, 1\right)=\left(\begin{array}{c}
-1 \\
p^{-1} T^{-1}
\end{array}\right) \cdot(1, p T)
$$

For the restriction of $f^{\prime}$ we obtain

$$
f^{\prime}=\operatorname{tr}\left(j \cdot g \cdot\left(\begin{array}{c}
-1 \\
p^{-1} T^{-1}
\end{array}\right) \cdot(1, p T) \cdot g^{-1}\right) .
$$

Let $g=\left(\begin{array}{ll}a & b \\ c & d\end{array}\right) \in w G L_{2}\left(\mathbb{Z}_{p}\right) w^{-1}$. Then

$$
g \cdot\left(\begin{array}{c}
-1 \\
p^{-1} T^{-1}
\end{array}\right)=\left(\begin{array}{c}
-a+p^{-1} b T^{-1} \\
-c+p^{-1} d T^{-1}
\end{array}\right)=\left(a-p^{-1} b T^{-1}\right) \cdot\left(\begin{array}{c}
-1 \\
\frac{-c+p^{-1} d T^{-1}}{a-p^{-1} b T^{-1}}
\end{array}\right)
$$

The second component of the last vector is equal to

$$
p^{-1} \cdot\left(\frac{a T-p^{-1} b}{-p c T+d}\right)^{-1}=p^{-1} g_{*}^{\prime-1}(T)^{-1} \text {. }
$$

Here

$$
g^{\prime}=w g w^{-1}=\left(\begin{array}{cc}
d & p c \\
p^{-1} b & a
\end{array}\right) \in G L_{2}\left(\mathbb{Z}_{p}\right)
$$

Similarly,

$$
(1, p T) \cdot g^{-1}=\operatorname{det}(g)^{-1} \cdot(d-p c T) \cdot\left(1, p \cdot g_{*}^{\prime-1}(T)\right)
$$


Putting

$$
u=a-p^{-1} b \cdot T^{-1}, \quad v=d-p c T
$$

we therefore obtain

$$
f^{\prime}=\operatorname{det}(g)^{-1} u v \cdot g_{*}^{\prime-1}(f)
$$

Of course, after reversing the identification of $\hat{\Omega}_{\left[\Lambda_{0}\right]}$ with $\hat{\Omega}_{\left[\Lambda_{1}\right]}$, the isomorphism $g_{*}^{\prime-1}$ becomes $g_{*}^{-1}$. We define

$$
\alpha=\operatorname{det}(g)^{-1} \cdot u^{-1} v^{-1}, \beta_{0}=\operatorname{det}(g) \cdot u^{-2}, \beta_{1}=\operatorname{det}(g) \cdot v^{-2}
$$

and the proof proceeds as before.

We now use the previous lemmas to glue the complexes on the open charts $\hat{\Omega}_{\Delta}$ with $p t_{\Delta} \in Z(j)$. Let $g_{1}, g_{2} \in G\left(\mathbb{Q}_{p}\right)^{0}$ such that $g_{i} \cdot p t_{\Delta_{0}} \in Z(j)$ for $i=1,2$ and with $g_{1} \Delta_{0} \cap g_{2} \Delta_{0}=\{[\Lambda]\}$. We define an isomorphism of complexes on $\hat{\Omega}_{[\Lambda]}$

$$
\mu_{g_{2}, g_{1}}: K\left(g_{1}, j\right)_{\mid \hat{\Omega}_{[\Lambda]}} \longrightarrow K\left(g_{2}, j\right)_{\mid \hat{\Omega}_{[\Lambda]}}
$$

by putting

$$
\mu_{g_{2}, g_{1}}=g_{2 *} \circ \mu_{h}^{0} \circ g_{1 *}^{-1} .
$$

Here $g_{2}=g_{1} \cdot h$ where $h \in G L_{2}\left(\mathbb{Z}_{p}\right)$ if $[\Lambda]$ is in the $G\left(\mathbb{Q}_{p}\right)^{0}$-orbit of $\left[\Lambda_{0}\right]$ and $h \in w G L_{2}\left(\mathbb{Z}_{p}\right) w^{-1}$ if $[\Lambda]$ is in the $G\left(\mathbb{Q}_{p}\right)^{0}$-orbit of $\left[\Lambda_{1}\right]$. The isomorphism $\mu_{h}^{0}$ has been defined in Lemma (5.3) resp. in Lemma (5.4). Again there is a transitivity condition. If $g_{3}$ is a third element with $g_{3} \cdot p t_{\Delta_{0}} \in Z(j)$ and with $g_{3} \Delta_{0} \cap g_{i} \Delta_{0}=$ $\{[\Lambda]\}, i=1,2$, then

$$
\mu_{g_{3}, g_{1}}=\mu_{g_{3}, g_{2}} \circ \mu_{g_{2}, g_{1}}
$$

Having constructed a global resolution of $\mathcal{O}_{Z(j)}$ on the complement of the isolated ordinary points of $Z(j)$ we now consider tensor products of two of them. We let $Z\left(j_{1}\right), Z\left(j_{2}\right)$ and $Z\left(j_{1}^{\prime}\right), Z\left(j_{2}^{\prime}\right)$ be two pairs of special cycles. We assume that

$$
\left(j_{1}, j_{2}\right) \cdot \gamma=\left(j_{1}^{\prime}, j_{2}^{\prime}\right)
$$

for

$$
\gamma=\left(\begin{array}{ll}
a & b \\
c & d
\end{array}\right) \in M_{2}\left(\mathbb{Z}_{p}\right)
$$

Let us first assume that $p t_{\Delta_{0}} \in Z\left(j_{1}\right) \cap Z\left(j_{2}\right)$ and $p t_{\Delta_{0}} \in Z\left(j_{1}^{\prime}\right) \cap Z\left(j_{2}^{\prime}\right)$. We will denote by $K(1, \underline{j})$ the tensor product $K\left(1, j_{1}\right) \otimes_{\mathcal{O}} K\left(1, j_{2}\right)$ with its bigrading, and similarly for $K\left(1, \underline{j}^{\prime}\right)$. We denote the differentials in degree -1 of $K\left(1, j_{i}\right)$ by $\partial_{i}$ and those of $K\left(1, j_{i}^{\prime}\right)$ by $\partial_{i}^{\prime}$. All differentials in degree -2 are identical and will be denoted by $\partial$. 
Lemma 5.5. There is a homomorphism of complexes

$$
\varphi: K\left(1, \underline{j}^{\prime}\right) \longrightarrow K(1, \underline{j})
$$

which is given degree by degree as follows.

in degree 0 :

$$
\varphi^{0}=\operatorname{id}_{\mathcal{O}}: \mathcal{O} \longrightarrow \mathcal{O}
$$

in degree $-1=(-1,0)+(0,-1)$ :

$$
\begin{array}{cccc}
\varphi^{-1}:(\mathcal{O} \oplus \mathcal{O}) \oplus(\mathcal{O} \oplus \mathcal{O}) & \longrightarrow & (\mathcal{O} \oplus \mathcal{O}) \oplus(\mathcal{O} \oplus \mathcal{O}) \\
(x, y) & \longmapsto & (x, y) \cdot{ }^{t} \gamma
\end{array}
$$

(here, as before, $x$ and $y$ are considered as column vectors).

in bi-degree $(-2,0)+(0,-2)$ :

$$
\begin{aligned}
\varphi^{-2}: \mathcal{O} \oplus \mathcal{O} & \longrightarrow \mathcal{O} \oplus \mathcal{O} \\
(x, y) & \longmapsto(x, y) \cdot{ }^{t} \gamma
\end{aligned}
$$

in bi-degree $(-1,-1)$ :

$$
\begin{array}{cccc}
\varphi^{-2}:(\mathcal{O} \oplus \mathcal{O}) \otimes(\mathcal{O} \oplus \mathcal{O}) & \longrightarrow & \mathcal{O} \oplus(\mathcal{O} \oplus \mathcal{O}) \otimes(\mathcal{O} \oplus \mathcal{O}) \oplus \mathcal{O} \\
(x \otimes y) & \longmapsto & \left(a b \psi_{1}(x, y), a d(x \otimes y)-b c(y \otimes x), c d \psi_{2}(x, y)\right)
\end{array}
$$

Here $\psi_{1}(x, y) \in \mathcal{O}$ is the unique element of bidegree $(-2,0)$ whose image under the differential $\partial$ of $K\left(1, j_{1}\right)$ is

$$
\partial \psi_{1}(x, y)=\partial_{1}(x) y-\partial_{1}(y) x
$$

The element $\psi_{2}(x, y)$ of bidegree $(0,-2)$ is similarly defined.

in degree $-3=(-2,-1)+(-1,-2)$ :

$$
\begin{aligned}
\varphi^{-3}:(\mathcal{O} \oplus \mathcal{O}) \oplus(\mathcal{O} \oplus \mathcal{O}) & \longrightarrow(\mathcal{O} \oplus \mathcal{O}) \oplus(\mathcal{O} \oplus \mathcal{O}) \\
(x, y) & \longmapsto(a d x+b c y, a d y+b c x)
\end{aligned}
$$

in degree $-4=(-2,-2)$ :

$$
\varphi^{-4}=(a d+b c) \mathrm{id}: \mathcal{O} \longrightarrow \mathcal{O}
$$


Proof. We have to check that $\varphi$ is indeed a homomorphism of complexes. We picture the complexes written horizontally and the homomorphism $\varphi$ vertically from the north to the south.

in degree -1 : Via the NE-passage we have

$$
(x, y) \longmapsto \partial_{1}^{\prime}(x)+\partial_{2}^{\prime}(y) \longmapsto \partial_{1}^{\prime}(x)+\partial_{2}^{\prime}(y)
$$

However, and this is the crucial observation for the proof, the differential in degree -1 of $K(1, j)$ depends linearly on $j$. Hence

$$
\partial_{1}^{\prime}=a \partial_{1}+c \partial_{2} \quad, \quad \partial_{2}^{\prime}=b \partial_{1}+d \partial_{2}
$$

Therefore the last expression is equal to

$$
\partial_{1}(a x+b y)+\partial_{2}(c x+d y)=\partial^{-1}\left(\varphi^{-1}(x, y)\right)
$$

where $\partial^{-1}$ is the differential in degree -1 of $K(1, \underline{j})$.

in degree -2 : The argument in degree $(-2,0)+(0,-2)$ is identical. Let us consider an element $x \otimes y$ of bi-degree $(-1,-1)$. It is mapped via the NE route to

$$
x \otimes y \mapsto \partial_{1}^{\prime}(x) y-\partial_{2}^{\prime}(y) x \mapsto\left(-a \partial_{2}^{\prime}(y) x+b \partial_{1}^{\prime}(x) y,-c \partial_{2}^{\prime}(y) x+d \partial_{1}^{\prime}(x) y\right)
$$

Inserting the expression (5.43) for $\partial_{1}^{\prime}$ and $\partial_{2}^{\prime}$ and collecting terms, this last element is equal to

$$
\left(a b \partial\left(\psi_{1}(x, y)\right)+b c \partial_{2}(x) y-a d \partial_{2}(y) x, c d \partial\left(\psi_{2}(x, y)\right)+a d \partial_{1}(x) y-b c \partial_{1}(y) x\right)
$$

Via the SW route the element goes to

$$
\begin{gathered}
x \otimes y \mapsto\left(a b \psi_{1}(x, y), a d(x \otimes y)-b c(y \otimes x), c d \psi_{2}(x, y)\right) \mapsto \\
\mapsto\left(a b \partial\left(\psi_{1}(x, y)\right)-a d \partial_{2}(y) x+b c \partial_{2}(x) y,\right. \\
\left.c d \psi_{2}(x, y)+a d \partial_{1}(x) y-b c \partial_{1}(y) x\right),
\end{gathered}
$$

which proves the claim in this case.

in degree $-3=(-2,-1)+(-1,-2)$ : Let $(x, 0)$ be of bi-degree $(-2,-1)$. Then via the NE route this element goes to

$$
\begin{aligned}
& (x, 0) \mapsto\left(\partial_{2}^{\prime}(x), \partial(1) \otimes x, 0\right) \mapsto \\
& \mapsto\left(a \partial_{2}^{\prime}(x)+a b \psi_{1}(\partial(1), x), a d(\partial(1) \otimes x)-b c(x \otimes \partial(1)), c \partial_{2}^{\prime}(x)+c d \psi_{2}(\partial(1), x)\right)
\end{aligned}
$$


The entry of bi-degree $(-2,0)$ is equal to

$$
a b\left(\partial_{1}(x)+\psi_{1}(\partial(1), x)\right)+a d \partial_{2}(x)=a d \partial_{2}(x) .
$$

Here we used that the first summand vanishes since

$$
\partial\left(\partial_{1}(x)+\psi_{1}(\partial(1), x)\right)=\partial_{1}(x) \cdot \partial(1)+\partial^{2}(1) x-\partial(1) \partial_{1}(x)=0 .
$$

The same reasoning shows that the entry of bi-degree $(0,-2)$ is equal to $b c \partial_{1}(x)$. On the other hand, the image of $(x, 0)$ via the SW route is equal to

$$
(x, 0) \mapsto(a d x, b d x) \mapsto\left(a d \partial_{2}(x), a d(\partial(1) \otimes x)-b c(x \otimes \partial(1)), b c \partial_{1}(x)\right),
$$

which shows the claim for elements of bi-degree $(-2,-1)$. The case of elements of bi-degree $(-1,-2)$ is analogous.

in degree $-4:$ Here the generator $1 \in \mathcal{O}$ is sent via the NE route to

$$
1 \mapsto(\partial(1), \partial(1)) \mapsto(a d \partial(1)+b c \partial(1), a d \partial(1)+b c \partial(1))
$$

Via the SW passage the same element is sent to

$$
1 \mapsto(a d+b c) \cdot 1 \mapsto(a d+b c)(\partial(1), \partial(1))
$$

which proves the claim in this case.

We note that the formulas for $\varphi$ do not involve the special endomorphisms $j_{1}, j_{2}, j_{1}^{\prime}, j_{2}^{\prime}$ and neither do the formulas for the glueing maps $\lambda_{g}^{0}, \mu_{g}^{0}$. This observation is crucial for the proof of the next lemma.

Lemma 5.6. a) Let $g$ be an element of the Iwahori subgroup corresponding to $\Delta_{0}$. The following diagram is commutative.

$$
\begin{array}{crr}
K\left(1, \underline{j}^{\prime}\right) & \stackrel{\varphi}{\longrightarrow} & K(1, \underline{j}) \\
\lambda_{g}^{\prime 0} \otimes \lambda_{g}^{\prime 0} \downarrow & \downarrow \lambda_{g}^{0} \otimes \lambda_{g}^{0} \\
K\left(1, g^{-1} \underline{j}^{\prime} g\right) & \stackrel{\varphi}{\longrightarrow} & K\left(1, g^{-1} \underline{j} g\right)
\end{array}
$$

b) Let $g \in G L_{2}\left(\mathbb{Z}_{p}\right)$. The following diagram of complexes on $\hat{\Omega}_{\left[\Lambda_{0}\right]}$ is commutative.

$$
\begin{aligned}
& K\left(1, \underline{j}^{\prime}\right)_{\mid \hat{\Omega}_{\left[\Lambda_{0}\right]}} \stackrel{\varphi}{\longrightarrow} \quad K(1, \underline{j})_{\mid \hat{\Omega}_{\left[\Lambda_{0}\right]}} \\
& \mu_{g}^{\prime 0} \otimes \mu_{g}^{\prime 0} \downarrow \quad \downarrow \mu_{g}^{0} \otimes \mu_{g}^{0} \\
& K\left(1, g^{-1} \underline{j}^{\prime} g\right)_{\mid \hat{\Omega}_{\left[\Lambda_{0}\right]}} \stackrel{\varphi}{\longrightarrow} K\left(1, g^{-1} \underline{j} g\right)_{\mid \hat{\Omega}_{\left[\Lambda_{0}\right]}}
\end{aligned}
$$


The analogous statement holds for $g \in w G L_{2}\left(\mathbb{Z}_{p}\right) w^{-1}$ and for the restrictions to $\hat{\Omega}_{\left[\Lambda_{1}\right]}$.

Proof. a) We again check this degree by degree. In degree 0 all homomorphisms are the identity, hence the assertion is obvious.

degree $-1=(-1,0)+(0,-1)$ : Via the NE passage an element $(x, y)$ is sent to

$$
(x, y) \mapsto(x, y) \cdot{ }^{t} \gamma \mapsto \beta \cdot\left(x^{\prime}, y^{\prime}\right) \cdot{ }^{t} \gamma
$$

Here for brevity we have set $x^{\prime}=g_{*}^{-1}(x)$. The result via the SW passage is obviously the same.

degree $(-2,0)+(0,-2)$ : Same argument as before.

bi-degree $(-1,-1)$ : Via the NE passage an element $x \otimes y$ is sent to

$$
\begin{aligned}
& \left.x \otimes y \mapsto\left(a b \psi_{1}(x, y)\right), a d(x \otimes y)-b c(y \otimes x), c d \psi_{2}(x, y)\right) \\
& \mapsto\left(\alpha \cdot a b \psi_{1}(x, y)^{\prime}, a d(\beta \otimes \beta)\left(x^{\prime} \otimes y^{\prime}\right)-b c(\beta \otimes \beta)\left(y^{\prime} \otimes x^{\prime}\right), \alpha \cdot c d \psi_{2}(x, y)^{\prime}\right) .
\end{aligned}
$$

Via the SW passage the element is sent to

$x \otimes y \mapsto\left(\beta x^{\prime}, \beta y^{\prime}\right) \mapsto\left(a b \psi_{1}\left(\beta x^{\prime}, \beta y^{\prime}\right), a d\left(\beta x^{\prime} \otimes \beta y^{\prime}\right)-b c\left(\beta y^{\prime} \otimes \beta x^{\prime}\right), c d \psi_{2}\left(\beta x^{\prime}, \beta y^{\prime}\right)\right)$.

Obviously the terms of degree $(-1,-1)$ coincide. Let us consider the terms of degree $(-2,0)$, leaving aside the common factor $a b$. But

$$
\begin{aligned}
\partial\left(\alpha \cdot \psi_{1}(x, y)^{\prime}\right) & =\beta \cdot \partial\left(\psi_{1}(x, y)\right)^{\prime} \\
& =\beta \cdot\left(\partial_{1}^{\prime}(x) y-\partial_{2}^{\prime}(y) x\right)^{\prime} \\
& =\partial_{1}^{\prime}(x)^{\prime} \cdot \beta y^{\prime}-\partial_{2}^{\prime}(y)^{\prime} \beta x^{\prime} \\
& =\partial_{1}\left(\beta x^{\prime}\right) \beta y^{\prime}-\partial_{2}\left(\beta y^{\prime}\right) \beta x \\
& =\partial\left(\psi_{1}\left(\beta x^{\prime}, \beta y^{\prime}\right)\right),
\end{aligned}
$$

where we used twice that $\lambda_{g}^{0}$ is a homomorphism of complexes. It follows that the terms of degree $(-2,0)$ are identical. The same argument applies to the components of degree $(0,-2)$.

degree $(-2,-1)+(-1,-2)$ : Via the NE passage the element $(x, 0)$ of degree $(-2,-1)$ goes to

$$
(x, 0) \mapsto(a d x, b c x) \mapsto\left(a d \alpha \cdot \beta x^{\prime}, b c \alpha \cdot \beta x\right)
$$


Via the SW route this element is sent to

$$
(x, 0) \mapsto\left(\alpha \cdot \beta x^{\prime}, 0\right) \mapsto\left(a d \alpha \cdot \beta x^{\prime}, b c \alpha \cdot \beta x^{\prime}\right)
$$

which proves the claim in this case. Elements $(0, y)$ of degree $(-1,-2)$ are analogous.

degree $(-2,-2):$ The element 1 is sent via the NE passage to

$$
1 \mapsto(a d+b c) \cdot 1 \mapsto(a d+b c) \cdot \alpha
$$

and via the SW passage to

$$
1 \mapsto \alpha \mapsto(a d+b c) \alpha
$$

This concludes the proof of a). The proof of b) is formally identical.

We now drop the assumption that $p t_{\Delta_{0}} \in Z(\underline{j}) \cap Z\left(\underline{j}^{\prime}\right)$. Let $\Delta$ be an edge with $p t_{\Delta} \in Z(j) \cap Z\left(j^{\prime}\right)$. Let $g \in G\left(\mathbb{Q}_{p}\right)^{0}$ with $\Delta=g \Delta_{0}$. Then, just as we used the isomorphisms $\lambda_{h}^{0}$ (for $h$ lying in the Iwahori subgroup) to construct well-defined complexes on the open chart $\hat{\Omega}_{\Delta}$, we use part a) of the previous lemma to define homomorphisms of complexes on $\hat{\Omega}_{\Delta}$,

$$
\varphi_{g}: K\left(g, \underline{j}^{\prime}\right) \longrightarrow K(g, \underline{j})
$$

by putting

$$
\varphi_{g}=g_{*} \circ \varphi \circ g_{*}^{-1}
$$

If $g_{1} \Delta_{0}=g_{2} \Delta_{0}=\Delta$, then by part a) of the lemma we have

$$
\varphi_{g_{2}} \circ \lambda_{g_{2}, g_{1}}^{\prime}=\lambda_{g_{2}, g_{1}} \circ \varphi_{g_{1}}: K\left(g, \underline{j}^{\prime}\right) \longrightarrow K\left(g_{2}, \underline{j}\right)
$$

which means that the homomorphisms on the local chart $\hat{\Omega}_{\Delta}$ are well-defined. By part b) of the previous lemma the homomorphisms just constructed glue on the overlaps of the open charts in question.

Lemma 5.7. Suppose with the above notations that $\gamma \in G L_{2}\left(\mathbb{Z}_{p}\right)$ and that $a d+b c$ is a unit, i.e., equivalently, that $(a d)^{2}-(b c)^{2} \in \mathbb{Z}_{p}^{\times}$. Then the homomorphism of complexes constructed above is an isomorphism.

Proof. It suffices to check this for $\varphi: K\left(1, \underline{j}^{\prime}\right) \rightarrow K(1, \underline{j})$, i.e., to show that the determinant of $\varphi$ is a unit in each degree. 
in degree $0: \operatorname{det}\left(\varphi^{0}\right)=1$

in degree $-1: \operatorname{det}\left(\varphi^{-1}\right)=\operatorname{det}(\gamma)$

in degree $-2: \operatorname{det}\left(\varphi^{2}\right)=\operatorname{det}(\gamma)^{2} \cdot\left((a d)^{2}-(b c)^{2}\right)$

in degree $-3: \operatorname{det}\left(\varphi^{-3}\right)=\operatorname{det}(\gamma)^{2} \cdot(a d+b c)^{2}$

in degree $-4: \operatorname{det}\left(\varphi^{-4}\right)=a d+b c$.

Proof of Theorem 5.1. By hypothesis we have the relation (5.40), where $\gamma \in$ $G L_{2}\left(\mathbb{Z}_{p}\right)$. If $a d+b c \in \mathbb{Z}_{p}^{\times}$the result follows from the previous lemma. Otherwise we write $\gamma=\gamma_{1} \ldots \gamma_{r}$ as a product of elements which satisfy the hypothesis of this lemma and obtain the desired isomorphism as the composition of the isomorphisms of the previous lemma

$$
K\left(1, \underline{j}^{\prime}\right)=K(1, \underline{j} \cdot \gamma) \stackrel{\varphi_{r}}{\longrightarrow} K\left(1, \underline{j} \cdot \gamma_{1} \ldots \gamma_{r-1}\right) \longrightarrow \ldots \stackrel{\varphi_{1}}{\longrightarrow} K(1, \underline{j})
$$

Example 5.8. We give an example to illustrate that in the situation of Theorem 5.1 the relative positions of the cycles $Z\left(j_{1}\right)$ and $Z\left(j_{2}\right)$ resp. of $Z\left(j_{1}^{\prime}\right)$ and $Z\left(j_{2}^{\prime}\right)$ can differ radically. Assume that $j_{1}, j_{2}$ is a diagonal basis with

$$
q\left(j_{1}\right)=\varepsilon_{1} \quad(\text { thus } \alpha=0), \text { and } q\left(j_{2}\right)=\varepsilon_{2} \cdot p^{\beta},
$$

with $\beta>0$ even and $\chi\left(\varepsilon_{1}\right)=-1$. Then $Z\left(j_{1}\right)$ is simply a horizontal divisor isomorphic to the disjoint sum of two copies of $\operatorname{Spf} W$ meeting the special fibre in two ordinary special points $x_{1}, x_{2}$ of $\mathbb{P}_{[\Lambda]}^{1}$, where $[\Lambda]$ is the unique vertex fixed by $j_{1}$. This vertex is also fixed by $j_{2}$ and the component $\mathbb{P}_{[\Lambda]}^{1}$ occurs with multiplicity $\beta / 2$ in $Z\left(j_{2}\right)$. In particular $(Z(\mathbf{j}))_{\text {red }}=\left\{x_{1}, x_{2}\right\}$ and

$$
\left(Z\left(j_{1}\right), Z\left(j_{2}\right)\right)=\beta
$$

As a new basis of $\mathbf{j}$ let us take

$$
j_{1} \text {, and } j_{2}^{\prime}=j_{2}+\lambda j_{1}, \lambda \in \mathbb{Z}_{p}^{\times} .
$$

Then

$$
q\left(j_{2}^{\prime}\right)=\lambda^{2} \varepsilon_{1}+\varepsilon_{2} \cdot p^{\beta}=\lambda^{2} \varepsilon_{1}\left(1+\lambda^{-2} \varepsilon_{1}^{-1} \varepsilon_{2} \cdot p^{\beta}\right) \in \mathbb{Z}_{p}^{\times}-\mathbb{Z}_{p}^{\times, 2}
$$


Hence $q\left(j_{2}^{\prime}\right)=\varepsilon_{2}^{\prime}$ with $\chi\left(\varepsilon_{2}^{\prime}\right)=-1$ and thus $Z\left(j_{2}^{\prime}\right)$ is, just as $Z\left(j_{1}\right)$, a horizontal divisor isomorphic to the disjoint sum of two copies of $\operatorname{Spf} W$ and meeting the special fibre in $x_{1}$ and $x_{2}$.

Let us calculate the intersection number $\left(Z\left(j_{1}\right), Z\left(j_{2}^{\prime}\right)\right)$ by using the local equations. Since $Z\left(j_{1}\right)$ and $Z\left(j_{2}^{\prime}\right)$ are coprime divisors, we only have to determine the lengths of the local rings at $x_{1}$ and $x_{2}$. Let us choose a basis for $\Lambda$ such that

$$
j_{1}=\left(\begin{array}{cc}
0 & 1 \\
\varepsilon_{1} & 0
\end{array}\right) \quad, \quad j_{2}=\left(\begin{array}{cc}
a & b \\
-\varepsilon_{1} b & -a
\end{array}\right) .
$$

Then $j_{2}^{\prime}=\left(\begin{array}{cc}a & b+\lambda \\ \varepsilon_{1}(\lambda-b) & -a\end{array}\right)$ and the local equations for $Z\left(j_{1}\right)$ and $Z\left(j_{2}^{\prime}\right)$ are respectively

$$
T^{2}-\varepsilon_{1}=0,(b+\lambda) T^{2}-2 a T-\varepsilon_{1}(\lambda-b)=0 .
$$

The affine ring of $Z\left(j_{1}\right) \cap Z\left(j_{2}^{\prime}\right)$ is therefore

$$
W\left[T,\left(T^{p}-T\right)^{-1}\right]^{\wedge} /\left(2 a T-2 \varepsilon_{1} b, T^{2}-\varepsilon_{1}\right)
$$

which is isomorphic to

$$
W /(a-\eta b) \oplus W /(a+\eta b)
$$

where $\eta$ is a square root of $\varepsilon_{1}$ in $W$. Therefore

$$
\begin{aligned}
\left(Z\left(j_{1}\right), Z\left(j_{2}^{\prime}\right)\right) & =\operatorname{ord}_{p}(a-\eta b)+\operatorname{ord}_{p}(a+\eta b) \\
& =\operatorname{ord}_{p}\left(a^{2}-\varepsilon_{1} b^{2}\right)=\operatorname{ord}_{p} q\left(j_{2}\right)=\beta,
\end{aligned}
$$

in accordance with (5.49). Of course, in this case the assertion of Theorem 5.1 is trivial since

$$
\mathcal{O}_{Z\left(j_{1}\right)} \otimes^{\mathbb{L}} \mathcal{O}_{Z\left(j_{2}\right)}=\mathcal{O}_{Z(\mathbf{j})}=\mathcal{O}_{Z\left(j_{1}\right)} \otimes^{\mathbb{L}} \mathcal{O}_{Z\left(j_{2}^{\prime}\right)}
$$

is concentrated in degree zero.

\section{$\S 6$. Computation of intersection numbers.}

In this section, we combine the information obtained so far and give an explicit expression for the intersection number $\left(Z(j), Z\left(j^{\prime}\right)\right)$. 
Theorem 6.1. Let $j$ and $j^{\prime}$ be special endomorphisms such that their $\mathbb{Z}_{p}$-span $\mathbf{j}=\mathbb{Z}_{p} j+\mathbb{Z}_{p} j^{\prime}$ is of rank 2 and nondegenerate for the quadratic form. Let

$$
T=\left(\begin{array}{cc}
q(j) & \frac{1}{2}\left(j, j^{\prime}\right) \\
\frac{1}{2}\left(j^{\prime}, j\right) & q\left(j^{\prime}\right)
\end{array}\right),
$$

and suppose that $T$ is $G L_{2}\left(\mathbb{Z}_{p}\right)$-equivalent to diag $\left(\varepsilon_{1} p^{\alpha}, \varepsilon_{2} p^{\beta}\right)$, where $0 \leq \alpha \leq \beta$. Then

$\left(Z(j), Z\left(j^{\prime}\right)\right)=e_{p}(T)=$

$$
=\alpha+\beta+1- \begin{cases}p^{\alpha / 2}+2 \frac{p^{\alpha / 2}-1}{p-1} & \text { if } \alpha \text { is even and } \chi\left(\varepsilon_{1}\right)=-1, \\ (\beta-\alpha+1) p^{\alpha / 2}+2 \frac{p^{\alpha / 2}-1}{p-1} & \text { if } \alpha \text { is even and } \chi\left(\varepsilon_{1}\right)=1, \\ 2 \frac{p^{(\alpha+1) / 2}-1}{p-1} & \text { if } \alpha \text { is odd. }\end{cases}
$$

By Theorem 5.1, we may assume that the elements $j$ and $j^{\prime}$ diagonalize the quadratic form, i.e., that

$$
T=\left(\begin{array}{ll}
\varepsilon_{1} p^{\alpha} & \\
& \varepsilon_{2} p^{\beta}
\end{array}\right),
$$

with $0 \leq \alpha \leq \beta$.

Recall that

$$
\begin{aligned}
\left(Z(j), Z\left(j^{\prime}\right)\right) & =\left(Z(j)^{\text {pure }}, Z\left(j^{\prime}\right)^{\text {pure }}\right) \\
& =\left(Z(j)^{h}, Z\left(j^{\prime}\right)^{h}\right)+\left(Z(j)^{h}, Z\left(j^{\prime}\right)^{v}\right)+\left(Z(j)^{v}, Z\left(j^{\prime}\right)^{h}\right)+\left(Z(j)^{v}, Z\left(j^{\prime}\right)^{v}\right),
\end{aligned}
$$

where

$$
Z(j)^{v}=\sum_{[\Lambda]} \operatorname{mult}_{[\Lambda]}(j) \cdot \mathbb{P}_{[\Lambda]},
$$

as in Proposition 4.5. Here we assume that $q(j)=\varepsilon_{1} p^{\alpha}, q\left(j^{\prime}\right)=\varepsilon_{2} p^{\beta}$ and that $j j^{\prime}=-j^{\prime} j$. Recall that

$$
\operatorname{mult}_{[\Lambda]}(j)=\max \left\{\alpha / 2-d\left([\Lambda], \mathcal{B}^{j}\right), 0\right\}
$$

We organize the calculation according to the cases in table (2.26), assuming from now on that $\alpha \leq \beta$.

First consider the quantity $\left(Z(j)^{h}, Z\left(j^{\prime}\right)^{h}\right)+\left(Z(j)^{h}, Z\left(j^{\prime}\right)^{v}\right)+\left(Z(j)^{v}, Z\left(j^{\prime}\right)^{h}\right)$. Recall, for example, that in the case $\alpha$ is even and $\chi\left(\varepsilon_{1}\right)=-1, Z(j)^{h}$ consists 
of two copies of $\operatorname{Spf}(W)$ meeting $\mathbb{P}_{[\Lambda(j)]}$, while, if $\alpha$ is odd, then $Z(j)^{h}$ consists of one copy of $\operatorname{Spf}\left(W^{\prime}\right)$ meeting $\operatorname{pt}_{\Delta(j)}$, where $\Delta(j)$ is the edge containing the fixed point of $j$. Otherwise $Z(j)^{h}$ is empty. Taking into account the multiplicites of the vertical components and Lemmas 4.8 and 4.9 , we obtain the following table of values of $\left(Z(j)^{h}, Z\left(j^{\prime}\right)^{h}\right)+\left(Z(j)^{h}, Z\left(j^{\prime}\right)^{v}\right)+\left(Z(j)^{v}, Z\left(j^{\prime}\right)^{h}\right)$ :

\begin{tabular}{|c|c|c|c|}
\hline$j \backslash j^{\prime}$ & $\begin{array}{c}\beta \text { even } \\
\chi\left(\varepsilon_{2}\right)=-1\end{array}$ & $\begin{array}{c}\beta \text { even } \\
\chi\left(\varepsilon_{2}\right)=1\end{array}$ & $\beta$ odd \\
\hline $\begin{array}{c}\alpha \text { even } \\
\chi\left(\varepsilon_{1}\right)=-1\end{array}$ & $\alpha+\beta$ & $\beta$ & $\emptyset$ \\
\hline $\begin{array}{c}\alpha \text { even } \\
\left(\varepsilon_{1}\right)=1\end{array}$ & $\alpha$ & 0 & $\alpha$ \\
\hline$\alpha$ odd & $\emptyset$ & $\beta$ & $\alpha+\beta-1$ \\
\hline
\end{tabular}

It then remains to calculate $\left(Z(j)^{v}, Z\left(j^{\prime}\right)^{v}\right)$, using Lemma 4.7. The first step is the following simple observation.

Lemma 6.2. For any vertex $[\Lambda] \in \mathcal{B}$, let $r=d\left([\Lambda], \mathcal{B}^{j^{\prime}}\right)$ be the distance to the fixed point set of $j^{\prime}$. Note that $[\Lambda]$ lies on the boundary of $\mathcal{T}\left(j^{\prime}\right)$ precisely when $r=\beta / 2$. Then

$$
\left(\mathbb{P}_{[\Lambda]}, Z\left(j^{\prime}\right)^{v}\right)= \begin{cases}1-p & \text { when } 1 \leq r \leq \beta / 2-1, \\ \chi\left(\varepsilon_{2}\right)-p & \text { when } r=0 \text { and } \beta \text { is even } \\ -p & \text { when } r=1 / 2 \text { and } \beta \text { is odd } \\ 1 & \text { when } r=\beta / 2, \\ 0 & \text { otherwise. }\end{cases}
$$

Proof. First suppose that $1 \leq r \leq \beta / 2-1$, so that there is a unique edge leading from $[\Lambda]$ along the geodesic from $[\Lambda]$ to $\mathcal{B}^{j^{\prime}}$, and there are $p$ edges at $[\Lambda]$ leading away from $\mathcal{B}^{j^{\prime}}$. The intersection of $\mathbb{P}_{[\Lambda]}$ with $Z\left(j^{\prime}\right)^{v}$ is thus

$$
(\beta / 2-r+1)-(p+1)(\beta / 2-r)+p(\beta / 2-r-1)=1-p,
$$

in this case. The other cases are similar. For example, if $r=1 / 2$ and $\beta$ is odd, then $[\Lambda]$ is one endpoint of the edge containing the unique fixed point of $j^{\prime}$. The multiplicity in $Z\left(j^{\prime}\right)^{v}$ of the component corresponding to each these two endpoints is $(\beta-1) / 2$. Thus, the intersection of $\mathbb{P}_{[\Lambda]}$ with $Z\left(j^{\prime}\right)^{v}$ is

$$
(\beta-1) / 2-(p+1)(\beta-1) / 2+p(\beta-3) / 2=-p .
$$


Finally, note that if $r=\beta / 2$, then the multiplicity of $\mathbb{P}_{[\Lambda]}$ in $Z\left(j^{\prime}\right)$ is zero, while the multiplicity of the component corresponding to the unique neighboring vertex closer to $\mathcal{B}^{j^{\prime}}$ is 1 .

Proof of Theorem 6.1. In all calculations, we will count, with multiplicity in $Z(j)$, the number of vertices $[\Lambda]$ in $\mathcal{T}(j)$ whose associated component has a fixed intersection number with $Z\left(j^{\prime}\right)$. Recall that we always assume that $\alpha \leq \beta$.

We begin with simplest case: $\alpha$ and $\beta$ even with $\chi\left(\varepsilon_{1}\right)=\chi\left(\varepsilon_{2}\right)=-1$. In this case, $\mathcal{T}(j) \cap \mathcal{T}\left(j^{\prime}\right)=\mathcal{T}(j)$ is just the $\alpha / 2$ ball around the vertex $[\Lambda(j)]=\left[\Lambda\left(j^{\prime}\right)\right]$ fixed by both $j$ and $j^{\prime}$. We have

$$
\begin{aligned}
\left(Z(j)^{v}, Z\left(j^{\prime}\right)^{v}\right) & =-(p+1) \alpha / 2+(1-p) \sum_{r=1}^{\alpha / 2-1}(\alpha / 2-r)(p+1) p^{r-1} \\
& =-(p+1) \frac{p^{\alpha / 2}-1}{p-1} .
\end{aligned}
$$

In the case $\alpha$ and $\beta$ even with $\chi\left(\varepsilon_{1}\right)=-1$ and $\chi\left(\varepsilon_{2}\right)=1, \mathcal{T}(j) \cap \mathcal{T}\left(j^{\prime}\right)=\mathcal{T}(j)$ is again just the $\alpha / 2$ ball around the vertex $[\Lambda(j)]$. Thus,

$$
\begin{aligned}
\left(Z(j)^{v}, Z\left(j^{\prime}\right)^{v}\right) & =-(p-1) \alpha / 2+(1-p) \sum_{r=1}^{\alpha / 2-1}(\alpha / 2-r)(p+1) p^{r-1} \\
& =\alpha-(p+1) \frac{p^{\alpha / 2}-1}{p-1}
\end{aligned}
$$

where the change from $\chi\left(\varepsilon_{2}\right)=-1$ to $\chi\left(\varepsilon_{2}\right)=1$ causes the change in the first term.

The case $\alpha$ and $\beta$ even with $\chi\left(\varepsilon_{1}\right)=1$ and $\chi\left(\varepsilon_{2}\right)=-1$ is more complicated. Here, the geodesic joining the fixed vertex $\left[\Lambda\left(j^{\prime}\right)\right]$ to any given vertex $[\Lambda]$ runs a distance $\ell$ along the fixed apartment $\mathcal{B}^{j}$ and then a distance $r$ away from the apartment. The vertices $[\Lambda]$ in $\mathcal{T}(j)$ with $\ell=0$ are all joined to $\left[\Lambda\left(j^{\prime}\right)\right]$ by a geodesic emanating along an edge outside of $\mathcal{B}^{j}$. The contribution of such vertices is:

$$
-(p+1) \alpha / 2+(1-p) \sum_{r=1}^{\alpha / 2-1}(\alpha / 2-r)(p-1) p^{r-1}=1-\alpha-p^{\alpha / 2} .
$$


If $1 \leq \ell \leq(\beta-\alpha) / 2$, then vertices $[\Lambda]$ with $r=\alpha / 2$ lie in $\mathcal{T}\left(j^{\prime}\right)$. Hence the contribution of vertices with $1 \leq \ell \leq(\beta-\alpha) / 2$ is

$$
2(1-p) \sum_{\ell=1}^{(\beta-\alpha) / 2}\left(\alpha / 2+\sum_{r=1}^{\alpha / 2-1}(\alpha / 2-r)(p-1) p^{r-1}\right)=(\alpha-\beta)\left(p^{\alpha / 2}-1\right) .
$$

Next, if $(\beta-\alpha) / 2<\ell<\beta / 2$, then the vertices $[\Lambda]$ with $r<\beta / 2$ lie strictly inside $\mathcal{T}\left(j^{\prime}\right)$, and contribute

$$
2(1-p) \sum_{\ell=(\beta-\alpha) / 2+1}^{\beta / 2-1}\left(\alpha / 2+\sum_{r=1}^{\beta / 2-\ell-1}(\alpha / 2-r)(p-1) p^{r-1}\right)=2 \alpha-4 \frac{p^{\alpha / 2}-1}{p-1}
$$

Finally, the vertices on the boundary of $\mathcal{T}\left(j^{\prime}\right)$ contribute

$$
\alpha+2 \sum_{\ell=(\beta-\alpha) / 2+1}^{\beta / 2-1}(\alpha / 2-(\beta / 2-\ell))(p-1) p^{\beta / 2-\ell-1}=2 \frac{p^{\alpha / 2}-1}{p-1} .
$$

Here the initial $\alpha$ is the contribution from the two points of intersection of the apartment $\mathcal{B}^{j}$ with the boundary of $\mathcal{T}\left(j^{\prime}\right)$, i.e., the two points with $\ell=\beta / 2$.

The set $\mathcal{T}(j) \cap \mathcal{T}\left(j^{\prime}\right)$ in the case $\alpha$ and $\beta$ even with $\chi\left(\varepsilon_{1}\right)=\chi\left(\varepsilon_{2}\right)=1$ is identical with the corresponding set in the previous case! The only change in the formulas occurs in the first term (6.4), which is now

$$
-(p-1) \alpha / 2+(1-p) \sum_{r=1}^{\alpha / 2-1}(\alpha / 2-r)(p-1) p^{r-1}=1-p^{\alpha / 2},
$$

due to the change in the contribution of the central vertex. In effect, the total in this case is $\alpha$ plus that of the previous case.

In the case $\alpha$ is even, $\chi\left(\varepsilon_{1}\right)=1$ and $\beta$ is odd, $\mathcal{B}^{j^{\prime}}$ is the midpoint of an edge in the apartment $\mathcal{B}^{j}$. Each vertex $[\Lambda]$ is joined to this fixed midpoint by a unique geodesic, which runs along the apartment $\mathcal{B}^{j}$ a distance $\ell+\frac{1}{2}$ and then a distance $r$ outside the apartment. The contribution of the vertices with $\ell=0$ is

$$
-p \alpha+2(1-p) \sum_{r=1}^{\alpha / 2-1}(\alpha / 2-r)(p-1) p^{r-1}=-\alpha-2\left(p^{\alpha / 2}-1\right),
$$

where the initial $-p \alpha$ is the contribution of the two endpoints of the edge containing the fixed vertex. When $1 \leq \ell \leq(\beta-\alpha-1) / 2$, we may travel a distance $r=\alpha / 2-1$ and remain strictly inside $\mathcal{T}\left(j^{\prime}\right)$. Thus such vertices contribute

$$
\sum_{\ell=1}^{(\beta-\alpha-1) / 2}\left(\alpha / 2+\sum_{r=1}^{\alpha / 2-1}(\alpha / 2-r)(p-1) p^{r-1}\right)=(\alpha-\beta+1)\left(p^{\alpha / 2}-1\right) .
$$


Similarly, the vertices strictly inside $\mathcal{T}\left(j^{\prime}\right)$ but with $\ell>(\beta-\alpha-1) / 2$ contribute

$$
2(1-p) \sum_{\ell=(\beta-\alpha+1) / 2}^{(\beta-3) / 2}\left(\alpha / 2+\sum_{r=1}^{(\beta-3) / 2-\ell}(\alpha / 2-r)(p-1) p^{r-1}\right)=2 \alpha-4 \frac{p^{\alpha / 2}-1}{p-1} .
$$

Finally, the vertices on the boundary of $\mathcal{T}\left(j^{\prime}\right)$ contribute

$$
\alpha+2 \sum_{\ell=(\beta-\alpha+1) / 2}^{(\beta-3) / 2}(\alpha / 2-((\beta-1) / 2-\ell))(p-1) p^{(\beta-1) / 2-\ell-1}=2 \frac{p^{\alpha / 2}-1}{p-1}
$$

Note that here the value $r=(\beta-1) / 2-\ell$ puts $[\Lambda]$ on the boundary.

The next case is $\alpha$ odd and $\beta$ even with $\chi\left(\varepsilon_{2}\right)=1$. Here the whole ball $\mathcal{T}(j)$ lies entirely inside the tube $\mathcal{T}\left(j^{\prime}\right)$ around the fixed apartment $\mathcal{B}^{j^{\prime}}$. The total contribution is

$$
2(1-p) \sum_{r=0}^{(\alpha-1) / 2}((\alpha-1) / 2-r) p^{r}=\alpha+1-2 \frac{p^{(\alpha+1) / 2}-1}{p-1},
$$

where $d\left([\Lambda], \mathcal{B}^{j^{\prime}}\right)=r+\frac{1}{2}$.

The last case is $\alpha$ and $\beta$ odd. Here $j$ and $j^{\prime}$ fix the same midpoint, and we get

$$
-p(\alpha-1)+2(1-p) \sum_{r=1}^{(\alpha-1) / 2}((\alpha-1) / 2-r) p^{r}=2-2 \frac{p^{(\alpha+1) / 2}-1}{p-1} .
$$

Here the term $-p(\alpha-1)$ comes from the pair of vertices of the edge containing the fixed vertex. Note that they have multiplicity $(\alpha-1) / 2$ in $Z(j)$.

In each case, we sum the various contributions to obtain the following table of values of $\left(Z(j)^{v}, Z\left(j^{\prime}\right)^{v}\right)$ :

\begin{tabular}{|c|c|c|c|}
\hline$j \backslash j^{\prime}$ & $\begin{array}{c}\beta \text { even } \\
\chi\left(\varepsilon_{2}\right)=-1\end{array}$ & $\begin{array}{c}\beta \text { even } \\
\chi\left(\varepsilon_{2}\right)=1\end{array}$ & $\beta$ odd \\
\hline $\begin{array}{c}\alpha \text { even } \\
\chi\left(\varepsilon_{1}\right)=-1\end{array}$ & $(\mathbf{i})$ & $(\mathbf{i i})$ & $\emptyset$ \\
\hline $\begin{array}{c}\alpha \text { even } \\
\chi\left(\varepsilon_{1}\right)=1\end{array}$ & $(\mathbf{i i i})$ & $\mathbf{( i v )}$ & $\mathbf{( v )}$ \\
\hline$\alpha$ odd & $\emptyset$ & $\mathbf{( v i )}$ & $\mathbf{( v i i )}$ \\
\hline
\end{tabular}


Here are the values:

$$
\begin{aligned}
(\mathbf{i}) & =(6.2)=-(p+1) \frac{p^{\alpha / 2}-1}{p-1} \\
(\mathbf{i i}) & =(6.3)=\alpha-(p+1) \frac{p^{\alpha / 2}-1}{p-1} \\
(\mathbf{i i i}) & =(6.4)+(6.5)+(6.6)+(6.7)=\beta+1-(\beta-\alpha+1) p^{\alpha / 2}-2 \frac{p^{\alpha / 2}-1}{p-1} \\
(\mathbf{i v}) & =(\text { iii })+\alpha=\alpha+\beta+1-(\beta-\alpha+1) p^{\alpha / 2}-2 \frac{p^{\alpha / 2}-1}{p-1} \\
(\mathbf{v}) & =(6.9)+(6.10)+(6.11)+(6.12)=\beta+1-(\beta-\alpha+1) p^{\alpha / 2}-2 \frac{p^{\alpha / 2}-1}{p-1} \\
(\mathbf{v i}) & =(6.13)=\alpha+1-2 \frac{p^{(\alpha+1) / 2}-1}{p-1} \\
(\mathbf{v i i}) & =(6.14)=2-2 \frac{p^{(\alpha+1) / 2}-1}{p-1}
\end{aligned}
$$

Adding these to the expressions in (6.1), we obtain the result claimed in the Theorem.

\section{$\S 7$. Intersection numbers and representation densities.}

In this section, we will express the intersection number $\left(Z(j), Z\left(j^{\prime}\right)\right)=e_{p}(T)$ given in Theorem 6.1 in terms of representation densities and their derivatives. The analogous result in the case of a prime of good reduction is contained in sections 8 and 14 (Proposition 14.6) of [15]. The result in the present case is somewhat more complicated, and this reflects, perhaps, the effect of bad reduction.

We begin by reviewing some facts about the representation densities of quadratic forms, based on section 8 of [15] and [20]. For simplicity, we assume that $p \neq 2$.

Recall that, for $S \in \operatorname{Sym}_{m}\left(\mathbb{Z}_{p}\right)$ and $T \in \operatorname{Sym}_{n}\left(\mathbb{Z}_{p}\right)$ with $\operatorname{det}(S) \neq 0$ and $\operatorname{det}(T) \neq 0$, the classical representation density is

$$
\alpha_{p}(S, T)=\lim _{t \rightarrow \infty} p^{-t n(2 m-n-1) / 2}\left|\left\{x \in M_{m, n}\left(\mathbb{Z} / p^{t} \mathbb{Z}\right) ; S[x]-T \in p^{t} \operatorname{Sym}_{m}\left(\mathbb{Z}_{p}\right)\right\}\right| .
$$

Let

$$
S_{r}=\left(\begin{array}{lll}
S & & \\
& 1_{r} & \\
& & -1_{r}
\end{array}\right)
$$


be the orthogonal sum of $S$ and a split space of dimension $2 r$. Then there is a rational function $A_{S, T}(X)$ of $X$ such that

$$
\alpha_{p}\left(S_{r}, T\right)=A_{S, T}\left(p^{-r}\right)
$$

The derivative of the representation density is then

$$
\begin{aligned}
\alpha_{p}^{\prime}(S, T) & =\left.\frac{\partial}{\partial X}\left(A_{S, T}(X)\right)\right|_{X=1} \\
& =\left.\frac{\partial}{\partial X}\left(\alpha_{p}\left(S_{r}, T\right)\right)\right|_{r=0}
\end{aligned}
$$

We will be concerned with the case $m=3$ and $n=2$. Let

$$
S=-\left(\begin{array}{lll}
1 & & \\
& 1 & \\
& & -1
\end{array}\right),
$$

and

$$
S^{\prime}=-\left(\begin{array}{ccc}
\eta & & \\
& p & \\
& & -\eta p
\end{array}\right)
$$

where $\eta \in \mathbb{Z}_{p}^{\times}$with $\chi(\eta)=(\eta, p)_{p}=-1$. Here $(a, b)_{p}$ is the quadratic Hilbert symbol for $\mathbb{Q}_{p}$. Note that the form $S$ (resp. $S^{\prime}$ ) is the matrix, with respect to a suitable basis, of the restriction of the norm form of $M_{2}\left(\mathbb{Q}_{p}\right)$ (resp. the division quaternion algebra $B)$ to the integral trace zero elements $V\left(\mathbb{Z}_{p}\right)\left(\operatorname{resp} . V^{\prime}\left(\mathbb{Z}_{p}\right)\right)$. In particular,

$$
1=\operatorname{det}(S)=\operatorname{det}\left(S^{\prime}\right) \in \mathbb{Q}_{p}^{\times} / \mathbb{Q}_{p}^{\times, 2}
$$

and $S$ and $S^{\prime}$ have opposite Hasse invariants

$$
\varepsilon_{p}(S)=-\varepsilon_{p}\left(S^{\prime}\right)=1
$$

Note that this corresponds to the choice $\kappa=-1$ in section 8 of [15].

Remark: In earlier sections we have taken the quadratic form on $V\left(\mathbb{Q}_{p}\right)$ given by $x^{2}=q(x) \cdot 1_{2}$, so that $q(x)=-\nu(x)$, wher $\nu$ is the restriction of the reduced norm to $V$. In [15], the quadratic form $Q(x)=\nu(x)$ was used. Thus, to make a consistent link with results of [15] we use in this section and in section 9 below 
$Q(x)$ rather than $q(x)$. This change in convention introduces slightly awkward signs at several points.

Also let

$$
S^{\prime \prime}=-\left(\begin{array}{lll}
1 & & \\
& p & \\
& & -p
\end{array}\right)
$$

so that $S^{\prime \prime}$ is the matrix for the restriction of the norm form to the trace 0 elements in the lattice

$$
\left\{\left(\begin{array}{cc}
a & b \\
c & d
\end{array}\right) \in M_{2}\left(\mathbb{Z}_{p}\right) ; c \equiv 0(p)\right\}
$$

The following Proposition summarizes results of Kitaoka, [11], [15], Corollary 8.4 and 8.5, in the case of $S$ and results of Myers, [18] and T. Yang, [20] in the case of $S^{\prime}$ and $S^{\prime \prime}$.

Proposition 7.1. Let $T \in \operatorname{Sym}_{2}\left(\mathbb{Z}_{p}\right)$ and suppose that $T$ is $G L_{2}\left(\mathbb{Z}_{p}\right)$-equivalent to the matrix $\operatorname{diag}\left(\varepsilon_{1} p^{\alpha}, \varepsilon_{2} p^{\beta}\right)$ with $\alpha \leq \beta$. Let

$$
\mu_{p}(T)= \begin{cases}\chi\left(-\varepsilon_{1} \varepsilon_{2}\right) & \text { if } \alpha \text { and } \beta \text { are odd } \\ \chi\left(-\varepsilon_{2}\right) & \text { if } \alpha \text { is odd and } \beta \text { is even } \\ \chi\left(-\varepsilon_{1}\right) & \text { if } \alpha \text { is even and } \beta \text { is odd } \\ 1 & \text { if } \alpha \text { and } \beta \text { are both even. }\end{cases}
$$

Then

$$
\alpha_{p}(S, T) \neq 0 \Longleftrightarrow \mu_{p}(T)=1,
$$

and

$$
\alpha_{p}\left(S^{\prime}, T\right) \neq 0 \Longleftrightarrow \mu_{p}(T)=-1 .
$$

(ii) (Kitaoka) If $\mu_{p}(T)=1$, then

$$
\alpha_{p}(S, T)=\left(1-p^{-2}\right) \cdot \begin{cases}p^{\alpha / 2}+2 \frac{p^{\alpha / 2}-1}{p-1} & \text { if } \alpha \text { and } \beta \text { are even } \\ (\beta-\alpha+1) p^{\alpha / 2}+2 \frac{p^{\alpha / 2}-1}{p-1} & \text { and } \chi\left(-\varepsilon_{1}\right)=-1 \\ & \text { if } \alpha \text { is even } \\ 2 \frac{p^{(\alpha+1) / 2}-1}{p-1} & \text { and } \chi\left(-\varepsilon_{1}\right)=1, \\ & \text { if } \alpha \text { is odd. }\end{cases}
$$


(iii) If $\mu_{p}(T)=-1$, then

$\alpha_{p}^{\prime}(S, T)=-\left(1-p^{-2}\right) \cdot\left\{\begin{array}{lc}\sum_{j=0}^{(\alpha-1) / 2}(\alpha+\beta-4 j) p^{j} & \text { if } \alpha \text { is odd. } \\ \sum_{j=0}^{\alpha / 2-1}(\alpha+\beta-4 j) p^{j}+\frac{1}{2}(\beta-\alpha+1) p^{\alpha / 2} & \text { if } \alpha \text { is even and } \beta \text { is odd. }\end{array}\right.$

(iv) (Myers) If $\mu_{p}(T)=-1$, then

$$
\alpha_{p}\left(S^{\prime}, T\right)=2(p+1)
$$

(iv) (Myers, Yang) If $\mu_{p}(T)=1$, then

$$
\begin{aligned}
& \alpha_{p}^{\prime}\left(S^{\prime}, T\right)=-(p+1)(\alpha+\beta+2) \\
&+2 p \cdot \begin{cases}p^{\alpha / 2}+2 \frac{p^{\alpha / 2}-1}{p-1} & \text { if } \alpha \text { and } \beta \text { are even } \\
(\beta-\alpha+1) p^{\alpha / 2}+2 \frac{p^{\alpha / 2}-1}{p-1} & \text { and } \chi\left(-\varepsilon_{1}\right)=-1\end{cases} \\
& \text { if } \alpha \text { is even } \\
& 2 \frac{p^{(\alpha+1) / 2}-1}{p-1} \text { and } \chi\left(-\varepsilon_{1}\right)=1,
\end{aligned}
$$

(v) (Yang) If $\mu_{p}(T)=1$, then

$$
1=\frac{p^{2}}{p^{2}-1} \alpha_{p}(S, T)-\frac{1}{2(p-1)} \alpha_{p}\left(S^{\prime \prime}, T\right)
$$

The last relation follows immediately from Corollary 8.4 in [20]. For an explanation of the dichotomy of (i), see Proposition 1.3 of [15].

The following striking relation is then evident, [20], Theorem 8.1:

Corollary 7.2. If $\mu_{p}(T)=1$, then

$$
\alpha_{p}^{\prime}\left(S^{\prime}, T\right)=-(p+1)(\alpha+\beta+2)+\frac{2 p^{3}}{p^{2}-1} \alpha_{p}(S, T) .
$$

Remark: Kitaoka gave an explicit formula for the representation density $\alpha_{p}(S, T)$ when $T$ is any binary form, $n=2$, and $S$ is unimodular, $m$ is arbitrary [11]. He also handled the case where $n=3$ and $m=4$ [12]. In his thesis, [18], B. Myers 
gave a formula for $\alpha_{p}(S, T)$ in the case of a binary form $T$ where, when diagonalized, the entries of $S$ have $\operatorname{ord}_{p} \leq 1$. This formula made it possible to compute the derivative $\alpha_{p}^{\prime}\left(S^{\prime}, T\right)$ above. The relation of Corollary 7.2 was first observed in the thesis of B. Myers, where the term $-(p+1)(\alpha+\beta+2)$ was erroneously given as $-(p+1)(\alpha+3 \beta+2)$ in certain cases. Using a new method, Tonghai Yang, [20], found an explicit formula for the representation density $\alpha_{p}(S, T)$ for $T$ a binary form and for $S$ an arbitrary form. His result (which includes the case $p=2$ ) thus allow the computation of any $\alpha_{p}^{\prime}(S, T)$ for a binary $T$. Recently, Katsurada, [10], gave an explicit (very complicated) formula for $\alpha_{p}(S, T)$ when $T$ is arbitrary and $S$ is a sum of hyperbolic planes. This should allow the computation of the derivatives $\alpha_{p}^{\prime}(S, T)$ in cases $m=2 \ell$ and $n=2 \ell-1$ in the interesting case in which $\alpha_{p}(S, T)=0$, cf. Proposition 1.3 and Theorem 6.1 of [15].

Comparing the expressions of Proposition 7.1 and Corollary 7.2 with the formula for the intersection number given in Theorem 6.1, we obtain the following result.

Theorem 7.3. As in Theorem 6.1, suppose that $j$ and $j^{\prime}$ are special endomorphisms with matrix of inner products $T$, defined using the quadratic form $Q .{ }^{2}$ Then, $\mu_{p}(T)=1$, and

$$
\left(Z(j), Z\left(j^{\prime}\right)\right)=e_{p}(-T)=-\frac{1}{p+1} \alpha_{p}^{\prime}\left(S^{\prime}, T\right)+\frac{2 p^{2}}{p+1} \alpha_{p}(S, T)+\frac{1}{2(p-1)} \alpha_{p}\left(S^{\prime \prime}, T\right) .
$$

Note that this identity holds for all $T$ and that the coefficients are independent of $T$, so that there is not as much flexibility as it might at first appear. Also, the occurrence of $e_{p}(-T)$ is due to our change in the sign of the quadratic form in this section.

As explained in [15], section 8 and Appendix, the representation densities and their derivatives are closely related to certain generalized Whittaker functions for the metaplectic cover of $S p_{2}\left(\mathbb{Q}_{p}\right)$. In our present case, let $\varphi_{p}$ be the characteristic function of $V\left(\mathbb{Z}_{p}\right)$, let $\varphi_{p}^{\prime}$ be the characteristic function of $V^{\prime}\left(\mathbb{Z}_{p}\right)$, and let $\varphi_{p}^{\prime \prime}$ be the characteristic function of the sublattice of $V\left(\mathbb{Z}_{p}\right)$ where $c$ is divisible by $p$. Also let $\Phi_{p}(s), \Phi_{p}^{\prime}(s)$, and $\Phi_{p}^{\prime \prime}(s)$ be the standard sections of the induced representation $I_{2, p}(s)$ whose values at $s=0$ are $\lambda_{p}\left(\varphi_{p}\right), \lambda_{p}^{\prime}\left(\varphi_{p}^{\prime}\right)$, and $\lambda_{p}\left(\varphi_{p}^{\prime \prime}\right)$ respectively. Then, as in Proposition A.6 of [15], the values of the associated

2 Thus $T$ in this Theorem is the negative of the matrix attached to $j$ and $j^{\prime}$ in previous sections, e.g., in Theorem 6.1. 
generalized Whittaker functions at integers $r \geq 0$ are

$$
\begin{aligned}
& W_{T, p}\left(e, r, \Phi_{p}\right)=\gamma_{p}\left(V_{p}\right) \cdot \alpha_{p}\left(S_{r}, T\right), \\
& W_{T, p}\left(e, r, \Phi_{p}^{\prime}\right)=p^{-2} \gamma_{p}\left(V_{p}^{\prime}\right) \cdot \alpha_{p}\left(S_{r}^{\prime}, T\right),
\end{aligned}
$$

and

$$
W_{T, p}\left(e, r, \Phi_{p}^{\prime \prime}\right)=p^{-2} \gamma_{p}\left(V_{p}\right) \cdot \alpha_{p}\left(S_{r}^{\prime \prime}, T\right) .
$$

Note that, by Proposition A.4 of [15],

$$
\gamma_{p}\left(V_{p}\right)=\gamma_{p}\left(V_{p}^{\prime}\right)=\gamma_{p}\left(-1, \psi_{p}\right)^{3}=1,
$$

for our standard $\psi_{p}$ and $p \neq 2$. In our case, since $\mu_{p}(T)=1$, the quantities of interest to us are then

$$
\begin{aligned}
& W_{T, p}^{\prime}\left(e, 0, \Phi_{p}^{\prime}\right)=-p^{-2} \log (p) \alpha_{p}^{\prime}\left(S^{\prime}, T\right), \\
& W_{T, p}\left(e, 0, \Phi_{p}\right)=\alpha_{p}(S, T),
\end{aligned}
$$

and

$$
W_{T, p}\left(e, 0, \Phi_{p}^{\prime \prime}\right)=p^{-2} \alpha_{p}\left(S^{\prime \prime}, T\right) .
$$

The relation of Theorem 7.3 can be expressed in terms of the derivative of a Whittaker function.

Corollary 7.4. Let $A(s)$ and $B(s)$ be rational functions of $p^{-s}$ satisfying

$$
A(0)=B(0)=0
$$

and

$$
A^{\prime}(0)=2 \log (p), \quad \text { and } \quad B^{\prime}(0)=\frac{1}{2} \frac{p+1}{p-1} \log (p) .
$$

Define a nonstandard section of $I_{2, p}(s)$ by

$$
\tilde{\Phi}_{p}(s)=\Phi_{p}^{\prime}(s)+A(s) \Phi_{p}(s)+B(s) \Phi_{p}^{\prime \prime}(s) .
$$

Then

$$
\frac{p+1}{p^{2}} \log (p) e_{p}(-T)=W_{T, p}^{\prime}\left(e, 0, \tilde{\Phi}_{p}\right)
$$

Note that, since the standard sections $\Phi_{p}(s), \Phi_{p}^{\prime}(s)$ and $\Phi_{p}^{\prime \prime}(s)$ are linearly independent at $s=0$, the conditions on $A(s)$ and $B(s)$ uniquely determine the first two terms of the Laurent expansion of $\tilde{\Phi}_{p}(s)$ at $s=0$.

For example, one possibility is

$$
A(s)=1-p^{-2 s} \quad \text { and } \quad B(s)=\frac{1}{4} \frac{1+p^{-1-s}}{1-p^{-1-s}}\left(1-p^{-2 s}\right) .
$$




\section{$\S 8$. Intersection numbers on Shimura curves.}

In this section we use the results about intersections of cycles in Drinfeld space obtained above to compute the contribution to the height pairing of certain 0cycles on Shimura curves of the intersections occuring in the fiber at $p$ for a prime $p$ which ramifies in the quaternion algebra. Our result extends that of [15] where the case of good reduction was considered. The notation will be slightly different from that of the earlier sections.

We begin by briefly reviewing the global moduli problem and the definition of cycles from section 14 of [15].

Let $B$ be an indefinite quaternion algebra over $\mathbb{Q}$ and let $D(B)$ be the product of the primes which ramify in $B$. Fix a maximal order $O_{B}$ in $B$. Let

$$
V=\left\{x \in B ; \operatorname{tr}^{0}(x)=0\right\},
$$

with quadratic form defined by $x^{2}=q(x) 1_{B}$, i.e., $q(x)=-\nu(x)$ where $\nu$ is the reduced norm. Note that we are using the negative of the form used in [15] and so the signature of this quadratic form is $(2,1)$. The action of $H=B^{\times}$ on $V$ by conjugation, $x \mapsto h x h^{-1}$, induces isomorphisms $H \simeq G \operatorname{Spin}(V)$ and $H / Z \simeq S O(V)$, where $Z$ is the center of $H$.

Fix a prime $p$ with $p \mid D(B)$, and a compact open subgroup $K \subset H\left(\mathbb{A}_{f}\right)$ of the form $K=K_{p} K^{p}$ with $K^{p} \subset H\left(\mathbb{A}_{f}^{p}\right)$ and $K_{p} \subset H\left(\mathbb{Q}_{p}\right)$. We assume that the image $K^{p} / Z^{p}$ of $K^{p}$ in $S O(V)\left(\mathbb{A}_{f}^{p}\right)$ is torsion free and that $K_{p}=\left(O_{B} \otimes \mathbb{Z}_{p}\right)^{\times}$.

Let $\mathcal{A}_{K}$ be the functor on $S c h_{\mathbb{Z}_{(p)}}$ which assigns to a scheme $S$ over $\mathbb{Z}_{(p)}$ the set of isomorphism classes of triples $(A, \iota, \bar{\eta})$ where

(i) $A$ is an abelian scheme over $S$, up to prime to $p$ isogeny,

(ii) $\iota: O_{B} \longrightarrow \operatorname{End}_{S}(A)$ is an embedding satisfying the special condition [21], p. 17 , and

(iii) $\bar{\eta}$ is an equivalence class, modulo right multiplication by $K^{p}$, of $O_{B}$ equivariant isomorphisms $\eta: \hat{V}^{p}(A) \stackrel{\sim}{\longrightarrow} B\left(\mathbb{A}_{f}^{p}\right)$, where

$$
\hat{V}^{p}(A)=\prod_{\ell \neq p} T_{\ell}(A) \otimes \mathbb{Q},
$$

is the rational Tate module of $A$.

We refer to [13] for the precise meaning of this last condition; in particular, if $S=\operatorname{Spec} k$ is the spectrum of a field, then the equivalence class $\bar{\eta}$ is stable under $\operatorname{Gal}(\bar{k} / k)$. 
As is well known, this functor is represented by a projective scheme over $\mathbb{Z}_{(p)}$ which we also denote by $\mathcal{A}_{K}$. The generic fiber $A_{K}=\mathcal{A}_{K} \times_{\text {Spec } \mathbb{Z}_{(p)}}$ Spec $\mathbb{Q}$ is the canonical model of the Shimura curve determined by $B$ and $K$.

Next, we recall the definition of special cycles. For $t \in \mathbb{Q}$, let

$$
V_{t}=\{x \in V ; q(x)=t\}
$$

be the corresponding hyperboloid. For a fixed negative integer $t \in \mathbb{Z}_{(p)}$ and a $K^{p}$-stable compact open subset $\omega \subset V\left(\mathbb{A}_{f}^{p}\right)$, we consider the functor $\mathcal{C}(t, \omega)$ which assigns to any $\mathbb{Z}_{(p)}$-scheme $S$ the set of isomorphism classes of collections $(A, \iota, \bar{\eta}, x)$, where $(A, \iota, \bar{\eta})$ is as before, and where

(iv) $x \in \operatorname{End}_{S}(A, \iota)$ is an endomorphism with $\operatorname{tr}^{0}(x)=0$ and such that, for any $\eta \in \bar{\eta}$, the endomorphism $\eta_{*}(x)$ of $B\left(\mathbb{A}_{f}^{p}\right)$ is given by right multiplication by an element of $\omega$.

This functor is representable. There is a natural morphism $\mathcal{C}(t, \omega) \rightarrow \mathcal{A}_{K}$ given by omitting the endomorphism $x$. This morphism is finite and unramified. On the generic fiber, this defines a 0 -cycle

$$
C(t, \omega):=\mathcal{C}(t, \omega) \times_{\operatorname{Spec}} \mathbb{Z}_{(p)} \operatorname{Spec} \mathbb{Q} \longrightarrow A_{K},
$$

described in more detail in section 10 of $[\mathbf{1 5}]$.

Let $\widehat{\mathcal{A}_{K}}$ be the formal completion of $\mathcal{A}_{K}$ along its special fiber, and let

$$
\hat{\mathcal{A}}=\left(\widehat{\mathcal{A}_{K}}\right)_{W}=\widehat{\mathcal{A}_{K}} \times{ }_{\operatorname{Spf} \mathbb{Z}_{p}} \operatorname{Spf} W
$$

be the base change of $\widehat{\mathcal{A}_{K}}$ to $W=W\left(\overline{\mathbb{F}}_{p}\right)$. The Drinfeld-Cherednik p-adic uniformization of $\hat{\mathcal{A}}$ is given as follows. Fix a base point $\xi_{0}=\left(A_{0}, \iota_{0}, \bar{\eta}_{0}\right) \in \hat{\mathcal{A}}\left(\overline{\mathbb{F}}_{p}\right)$, and let $B^{\prime}=\operatorname{End}^{0}\left(A_{0}, \iota_{0}\right)$. Then $B^{\prime}$ is a definite quaternion algebra over $\mathbb{Q}$ whose invariants agree with those of $B$ at all primes $\ell \neq p, \infty$. Let $H^{\prime}=B^{\prime, \times}$. Fix $\eta_{0} \in \overline{\eta_{0}}$. This choice induces an identification

$$
B^{\prime}\left(\mathbb{A}_{f}^{p}\right)=B\left(\mathbb{A}_{f}^{p}\right)^{\text {op }}
$$

determined by the condition $\eta_{0}\left(b^{\prime} v\right)=\eta_{0}(v) b$, and hence also an identification $H^{\prime}\left(\mathbb{A}_{f}^{p}\right)=H\left(\mathbb{A}_{f}^{p}\right)$, with order of multiplication reversed. Let $\mathbb{X}=A_{0}(p)$ be the p-divisible group of $A_{0}$. Then $\iota_{0}$ induces an action of $O_{B} \otimes \mathbb{Z}_{p}$ on $\mathbb{X}$ and, by the condition (ii) above, $\mathbb{X}$ is a s.f. $O_{B} \otimes \mathbb{Z}_{p}$ module over $k=\overline{\mathbb{F}}_{p}$. As in section 1 , we may also assume that 0 and 1 are critical indices of $\mathbb{X}$ and fix an identification $\operatorname{End}^{0}\left(\mathbb{X}, \iota_{0}\right)=M_{2}\left(\mathbb{Q}_{p}\right)$. This gives an identification $H^{\prime}\left(\mathbb{Q}_{p}\right)=G L_{2}\left(\mathbb{Q}_{p}\right)$. 
Recall from section 1 the category Nilp of $W$-schemes $S$ such that $p$ is locally nilpotent in $\mathcal{O}_{S}$ and the notation $\bar{S}=S \times_{\text {Spec } W} \operatorname{Spec} \overline{\mathbb{F}}_{p}$ for $S \in$ Nilp. Let $\hat{\mathcal{A}}^{\sim}$ be the functor on Nilp which associates to $S$ the isomorphism classes of collections $(A, \iota, \bar{\eta}, \psi)$ where $(A, \iota, \bar{\eta})$ is as before, and where

$$
\psi: A_{0} \times_{\mathrm{Spec} \overline{\mathbb{F}}_{p}} \bar{S} \longrightarrow A \times_{S} \bar{S}
$$

is an $O_{B}$-equivariant $p$-primary quasi-isogeny.

Let $\mathcal{M}^{\bullet}$ be the functor on Nilp which associates to $S \in$ Nilp the set of isomorphism classes of pairs $(X, \rho)$ where $X$ is a $p$-divisible group over $S$ and

$$
\rho: \mathbb{X} \times_{\text {Spec } \overline{\mathbb{F}}_{p}} \bar{S} \longrightarrow X \times{ }_{S} \bar{S}
$$

is a quasi-isogeny. Then $\mathcal{M}^{\bullet}$ is representable by a formal scheme and breaks up as a disjoint sum

$$
\mathcal{M}^{\bullet}=\coprod_{i \in \mathbb{Z}} \mathcal{M}^{i}
$$

where $\mathcal{M}^{i}$ is the locus where the height of $\rho$ is equal to $p^{i}$. We fix an element $\Pi \in B^{\prime}\left(\mathbb{Q}_{p}\right)^{\times}$such that $\operatorname{tr}^{0}(\Pi)=0$, and $\operatorname{ord}_{p}\left(\Pi^{2}\right)=1$. Using $\Pi$, we may identify $\mathcal{M}^{i}$ with $\mathcal{M}=\mathcal{M}^{0}$ (the Drinfeld space considered in section 1) via

$$
\mathcal{M} \stackrel{\sim}{\longrightarrow} \mathcal{M}^{i}, \quad(X, \rho) \mapsto\left(X, \rho \circ \Pi^{i}\right) .
$$

There is a natural morphism

$$
\hat{\mathcal{A}}^{\sim} \longrightarrow \mathcal{M}^{\bullet} \times H\left(\mathbb{A}_{f}^{p}\right) / K_{p}
$$

This morphism is defined as follows. Given $(A, \iota, \bar{\eta}, \psi) \in \hat{\mathcal{A}}^{\sim}(S)$, then passing to the corresponding $p$-divisible groups, we obtain a quasi-isogeny of $p$-divisible groups

$$
\rho(\psi): \mathbb{X} \times_{\text {Spec } \overline{\mathbb{F}}_{p}} \bar{S} \longrightarrow A(p) \times_{S} \bar{S}
$$

and $(A(p), \rho(\psi))$ is a point of $\mathcal{M}^{\bullet}(S)$. Also, for a choice of $\eta \in \bar{\eta}$ there is a unique element $g \in H\left(\mathbb{A}_{f}^{p}\right)$ for which the diagram

$$
\begin{array}{ccc}
\hat{V}^{p}\left(A_{0}\right) & \stackrel{\psi_{*}}{\longrightarrow} & \hat{V}^{p}(A) \\
\eta_{0} \downarrow & & \eta \downarrow \\
B\left(\mathbb{A}_{f}^{p}\right) & \stackrel{R_{g}}{\longrightarrow} & B\left(\mathbb{A}_{f}^{p}\right)
\end{array}
$$


commutes, and the coset $g K^{p}$ is uniquely determined by $\bar{\eta}$. Note, in (8.12), that $\hat{V}^{p}(A)=\hat{V}^{p}\left(A \times_{S} \bar{S}\right)$. An element $\gamma \in H^{\prime}(\mathbb{Q})$ acts on $\hat{\mathcal{A}}^{\sim}$ by changing the quasiisogeny $\psi$ to $\psi \circ \gamma^{-1}$. Since, for this change in $\psi$, the pair $\left((A(p), \rho(\psi)), g K^{p}\right)$ changes to $\left(\left(A(p), \rho(\psi) \circ \gamma^{-1}, \gamma^{-1} g K^{p}\right)\right.$, the map (8.10) is $H^{\prime}(\mathbb{Q})$-equivariant.

The theorem of Drinfeld-Cherednik asserts that passage to the quotient induces an isomorphism of formal schemes over $W,[\mathbf{5}],[\mathbf{1 9}],[\mathbf{2}]$ :

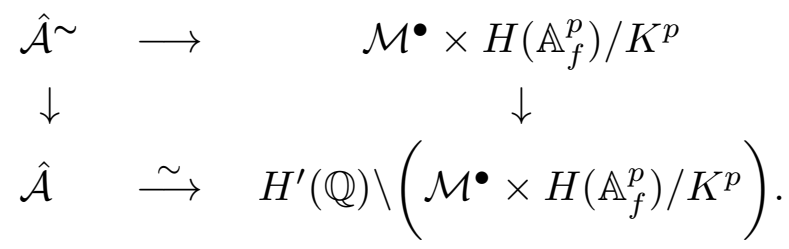

Now let

$$
V^{\prime}=\left\{x \in B^{\prime} ; \operatorname{tr}^{0}(x)=0\right\},
$$

with its quadratic form $q$, which we regard as the space of special endomorphisms of $A_{0}$ in $B^{\prime}=\operatorname{End}^{0}\left(A_{0}, \iota_{0}\right)$. Associating to any $x \in V^{\prime}(\mathbb{Q})$ the corresponding endomorphism of $\mathbb{X}=A_{0}(p)$, we obtain a special endomorphism $j=j(x)$ of $\mathbb{X}$, and in fact this induces an identification of $V^{\prime}\left(\mathbb{Q}_{p}\right)$ with the space of special endomorphisms of $\mathbb{X}$. Note that the notation has shifted; $V^{\prime}\left(\mathbb{Q}_{p}\right)$ was denoted by $V$ in the earlier sections. For $t \in \mathbb{Q}$, let

$$
V_{t}^{\prime}=\left\{x \in V^{\prime} ; q(x)=t\right\} .
$$

We may now relate the formal cycle $\hat{\mathcal{C}}:=\widehat{\mathcal{C}(t, \omega)} \times{ }_{\operatorname{Spf} \mathbb{Z}_{p}} \operatorname{Spf} W$ to the cycles in $\mathcal{M}$ considered earlier. Let $\hat{\mathcal{C}}^{\sim}=\hat{\mathcal{C}} \times{ }_{\hat{\mathcal{A}}} \hat{\mathcal{A}}^{\sim}$, so that a point of $\hat{\mathcal{C}}^{\sim}$ is a collection $(A, \iota, \bar{\eta}, x, \psi)$. From this data, we obtain an element $\psi^{*}(x) \in V_{t}^{\prime}(\mathbb{Q})$. By (8.10), we obtain a map

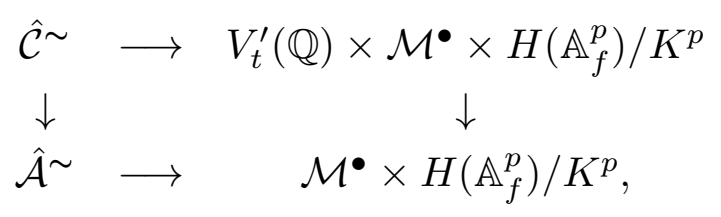

and, upon passage to the quotient, an injection

$$
\begin{array}{cccc}
\hat{\mathcal{C}} & \hookrightarrow & H^{\prime}(\mathbb{Q}) \backslash\left(V_{t}^{\prime}(\mathbb{Q}) \times \mathcal{M} \bullet \times H\left(\mathbb{A}_{f}^{p}\right) / K^{p}\right) \\
\downarrow & & \\
\hat{\mathcal{A}} & \stackrel{\sim}{\longrightarrow} & H^{\prime}(\mathbb{Q}) \backslash\left(\mathcal{M} \bullet \times H\left(\mathbb{A}_{f}^{p}\right) / K^{p}\right) .
\end{array}
$$


The image of this map is determined by the following incidence relations: the point $\left(x,(X, \rho), g K^{p}\right)$ lies in the image if and only if:

(i) $g^{-1} x g \in \omega$, and

(ii) for $j=j(x),(X, \rho) \in Z^{\bullet}(j)$.

Here $Z^{\bullet}(j)$ is the formal subscheme of $\mathcal{M}^{\bullet}$ defined by the obvious analogue of Definition 2.1.

Remark 8.1. Let $Z^{i}(j)=Z^{\bullet}(j) \cap \mathcal{M}^{i}$. Then, under the identification (8.9), we have

$$
Z\left(\Pi^{i} j \Pi^{-i}\right)=Z^{0}\left(\Pi^{i} j \Pi^{-i}\right) \stackrel{\sim}{\longrightarrow} Z^{i}(j)
$$

In particular, since $\Pi^{2}$ is central, we have

$$
Z(j) \simeq Z^{2 i}(j) \quad \text { and } \quad Z\left(j^{\vee}\right) \simeq Z^{2 i+1}(j)
$$

where $j^{\vee}=\Pi j \Pi^{-1}$.

Using the fact that $H^{\prime}(\mathbb{Q})$ acts transitively on $V_{t}^{\prime}(\mathbb{Q})$ when $t \neq 0$, we obtain an isomorphism

$$
\hat{\mathcal{C}} \stackrel{\sim}{\longrightarrow} H^{\prime}(\mathbb{Q})_{x} \backslash\left(Z^{\bullet}(j) \times I(x, \omega)\right),
$$

where $x$ is a fixed element of $V_{t}^{\prime}(\mathbb{Q}), j=j(x)$, and

$$
I(x, \omega)=\left\{g K^{p} \in H\left(\mathbb{A}_{f}^{p}\right) / K^{p} ; g^{-1} x g \in \omega\right\}
$$

Relation (8.20) can be viewed as giving a p-adic uniformization of the special cycle, analogous to the uniformization (8.13) of the whole space.

Remark 8.2. Assume that the generic fiber $C(t, \omega)=\mathcal{C}(t, \omega) \times_{\operatorname{Spec}} \mathbb{Z}_{(p)} \operatorname{Spec} \mathbb{Q}$ is nonempty. Then $t$ is represented by $V(\mathbb{Q})$. This follows from the fact that for any $\mathbb{C}$-valued point $(A, \iota, \bar{\eta})$ of $\mathcal{A}_{K}$ we have an injection

$$
\operatorname{End}^{0}(A, \iota) \hookrightarrow B
$$

Since $t$ is represented by $V(\mathbb{Q})$, it is a fortiori represented by $V\left(\mathbb{Q}_{p}\right)$. For the corresponding special endomorphism $j$ in $(8.20)$, this implies, since $B\left(\mathbb{Q}_{p}\right)$ is a quaternion division algebra, that $\mathbb{Q}_{p}(j)$ is a field, i.e., does not split. This is the global reason, referred to in Remark 3.5, for the observation in Corollary 3.4, that $Z(j)^{h}=\emptyset$ if $q(j)=\varepsilon p^{\alpha}$ with $\alpha$ even and $\chi(\varepsilon)=1$. 
Next, we consider the intersection of a pair of cycles $\mathcal{C}_{1}=\mathcal{C}\left(t_{1}, \omega_{1}\right)$ and $\mathcal{C}_{2}=$ $\mathcal{C}\left(t_{2}, \omega_{2}\right)$, following the procedure of section 3 of $[\mathbf{1 6}]$. We change notation and now denote by $\mathcal{C}$ the fiber product

$$
\mathcal{C}=\mathcal{C}\left(t_{1}, \omega_{1}\right) \times \mathcal{A}_{K} \mathcal{C}\left(t_{2}, \omega_{2}\right)
$$

Let $S$ be a connected scheme. For a point $\xi=\left(A_{\xi}, \iota, \bar{\eta}\right) \in \mathcal{A}_{K}(S)$, let $V_{\xi} \subset$ $\operatorname{End}_{S}^{0}\left(A_{\xi}, \iota\right)$ be the $\mathbb{Q}$-vector space of special endomorphisms (endomorphisms of trace 0$)$. This space has a $\mathbb{Q}$-valued quadratic form defined by $x^{2}=q_{\xi}(x) \cdot i d_{A}$. For a point $\xi=\left(A_{\xi}, \iota, \bar{\eta}, x_{1}, x_{2}\right)$ of $\mathcal{C}(S)$ the pair $x_{1}, x_{2}$ of elements of $V_{\xi}$ determine a symmetric matrix (the fundamental matrix associated to $\xi$ )

$$
T_{\xi}=\frac{1}{2}\left(\begin{array}{ll}
\left(x_{1}, x_{1}\right) & \left(x_{1}, x_{2}\right) \\
\left(x_{2}, x_{1}\right) & \left(x_{2}, x_{2}\right)
\end{array}\right)=\left(\begin{array}{cc}
t_{1} & \frac{1}{2}\left(x_{1}, x_{2}\right) \\
\frac{1}{2}\left(x_{2}, x_{1}\right) & t_{2}
\end{array}\right) \in \operatorname{Sym}_{2}(\mathbb{Q})
$$

where $(x, y)=q_{\xi}(x+y)-q_{\xi}(x)-q_{\xi}(y)$ is the bilinear form associated to $q_{\xi}$. Note that $\operatorname{det}\left(T_{\xi}\right)=t_{1} t_{2}-\frac{1}{4}\left(x_{1}, x_{2}\right)^{2}$. A basic fact is that $T_{\xi}$ is negative semi-definite. As observed in [16], the function $\xi \mapsto T_{\xi}$ is constant on each connected component of $\mathcal{C}$ and there is a decomposition

$$
\mathcal{C}=\coprod_{T} \mathcal{C}_{T}
$$

where, for $T \in \operatorname{Sym}_{2}(\mathbb{Q}), \mathcal{C}_{T}$ is the union of the components of $\mathcal{C}$ where $T_{\xi}=T$. Note that the only $T$ 's which actually contribute lie in $\operatorname{Sym}_{2}\left(\mathbb{Z}_{(p)}\right)$, are negative semi-definite, and have diagonal terms $t_{1}$ and $t_{2}$ as on the right side of (8.23). Since the signature of $V(\mathbb{Q})$ is $(2,1)$, and using an argument similar to that in Remark 8.2, we obtain:

Lemma 8.3. Suppose that $\operatorname{det}(T) \neq 0$ and hence that $T<0$. Then the image in $\mathcal{A}$ of the underlying point set of $\mathcal{C}_{T}$ lies in the special fiber. Moreover, $\mathcal{C}_{T}$ is proper over $\operatorname{Spec} \mathbb{Z}_{(p)}$.

The last statement follows from Corollary 2.13.

If $t_{1} t_{2} \notin \mathbb{Q}^{\times, 2}$, so that $\operatorname{det}(T) \neq 0$ for all $T$ appearing in the decomposition (8.24), then the discussion of section 4 provides a well defined intersection number $\left(\mathcal{C}_{1}, \mathcal{C}_{2}\right)$, cf. Remark 4.4. As a matter of fact, in that section, we only considered intersection numbers $\left(Z, Z^{\prime}\right)$ for closed subschemes of a regular two dimensional 
scheme $X$. The extension to the case of finite unramified morphisms $Z \rightarrow X$ and $Z^{\prime} \rightarrow X$ is immediate. In our case, we obtain

$$
\begin{aligned}
\left(\mathcal{C}_{1}, \mathcal{C}_{2}\right) & =\chi\left(\mathcal{C}, \mathcal{O}_{\mathcal{C}_{1}} \otimes^{\mathbb{L}} \mathcal{O}_{\mathcal{C}_{2}}\right) \\
& =\sum_{T} \chi\left(\mathcal{C}_{T}, \mathcal{O}_{\mathcal{C}_{1}} \otimes^{\mathbb{L}} \mathcal{O}_{\mathcal{C}_{2}}\right)
\end{aligned}
$$

If $t_{1} t_{2}$ is a square, then singular $T$ 's can occur in (8.24), and we define

$$
\left(\mathcal{C}_{1}, \mathcal{C}_{2}\right)^{\mathrm{ns}}:=\sum_{T, \operatorname{det}(T) \neq 0} \chi\left(\mathcal{C}_{T}, \mathcal{O}_{\mathcal{C}_{1}} \otimes^{\mathbb{L}} \mathcal{O}_{\mathcal{C}_{2}}\right)
$$

Our next goal is to compute the quantity $\chi\left(\mathcal{C}_{T}, \mathcal{O}_{\mathcal{C}_{1}} \otimes \mathbb{L} \mathcal{O}_{\mathcal{C}_{2}}\right)$ for a given nonsingular $T \in \operatorname{Sym}_{2}(\mathbb{Q})$. First we pass to the formal schemes $\hat{\mathcal{C}}_{1}, \hat{\mathcal{C}}_{2}$ and

$$
\hat{\mathcal{C}}=\hat{\mathcal{C}}_{1} \times \hat{\mathcal{A}}_{2}
$$

over $W$. Here

$$
\hat{\mathcal{C}}=\coprod_{T} \hat{\mathcal{C}}_{T}
$$

where $\hat{\mathcal{C}}$ (resp. $\hat{\mathcal{C}}_{T}$ ) is the base change to $W$ of the formal completion of $\mathcal{C}$ (resp. $\mathcal{C}_{T}$ ) along its special fiber. Passing to formal completions and making a formally étale base change leaves the intersection number unchanged, cf. Remark 4.4. Hence we obtain the following statement.

Lemma 8.4. Assume that $\operatorname{det}(T) \neq 0$. Then

$$
\chi\left(\mathcal{C}_{T}, \mathcal{O}_{\mathcal{C}_{1}} \otimes^{\mathbb{L}} \mathcal{O}_{\mathcal{C}_{2}}\right)=\chi\left(\hat{\mathcal{C}}_{T}, \mathcal{O}_{\hat{\mathcal{C}}_{1}} \otimes^{\mathbb{L}} \mathcal{O}_{\hat{\mathcal{C}}_{2}}\right)
$$

Let $\hat{\mathcal{C}}_{T}^{\sim}=\hat{\mathcal{C}}_{T} \times{ }_{\hat{\mathcal{A}}} \hat{\mathcal{A}}^{\sim}$, so that a point $\xi \in \hat{\mathcal{C}}_{T}^{\sim}(S)$ is a collection $\left(A_{\xi}, \iota, \bar{\eta}, x_{1}, x_{2}, \psi\right)$, where $\psi$ is a quasi-isogeny, as in (8.6). The special endomorphisms $x_{1}$ and $x_{2}$ of $A_{\xi}$ determine an ordered pair $\underline{x}=\left[\psi^{*}\left(x_{1}\right), \psi^{*}\left(x_{2}\right)\right]$ of special endomorphisms of $A_{0}$, and these, in turn, determine an ordered pair $\underline{j}=\left[j_{1}, j_{2}\right]$ of special endomorphisms of $\mathbb{X}$ and their $\mathbb{Z}_{p}$ span $\mathbf{j}=\mathbb{Z}_{p} j_{1}+\mathbb{Z}_{p} j_{2}$. Let

$$
V_{T}^{\prime, 2}=\left\{x \in V^{\prime, 2} ; \frac{1}{2}(x, x)=T\right\} .
$$


Then $\underline{x}$ lies in $V^{\prime}(\mathbb{Q})_{T}^{2}$. Thus, we obtain an inclusion

$$
\hat{\mathcal{C}}_{T}^{\sim} \hookrightarrow V^{\prime}(\mathbb{Q})_{T}^{2} \times \mathcal{M}^{\bullet} \times H\left(\mathbb{A}_{f}^{p}\right) / K^{p},
$$

analogous to (8.16). Again, the point $\left(\underline{x},(X, \rho), g K^{p}\right)$ lies in the image of this map if and only if:

(i) $g^{-1} \underline{x} g \in \omega_{1} \times \omega_{2}$, and

(ii) $(X, \rho) \in Z^{\bullet}(\mathbf{j})$, where $Z^{\bullet}(\mathbf{j})=Z^{\bullet}\left(j_{1}\right) \cap Z^{\bullet}\left(j_{2}\right)$.

Since $\operatorname{det}(T) \neq 0$, the group $H^{\prime}(\mathbb{Q})$ acts transitively on $V^{\prime}(\mathbb{Q})_{T}^{2}$, and the stabilizer of a fixed $\underline{x}$ is the center $Z^{\prime}(\mathbb{Q})$, which acts trivially on $V^{\prime}$. Letting

$$
I\left(\underline{x}, \omega_{1} \times \omega_{2}\right)=\left\{g K^{p} \in H\left(\mathbb{A}_{f}^{p}\right) ; g^{-1} \underline{x} g \in \omega_{1} \times \omega_{2}\right\}
$$

and passing to the quotient, we obtain an isomorphism

$$
\hat{\mathcal{C}}_{T} \stackrel{\sim}{\longrightarrow} Z^{\prime}(\mathbb{Q}) \backslash\left(Z \bullet(\mathbf{j}) \times I\left(\underline{x}, \omega_{1} \times \omega_{2}\right)\right) .
$$

Again, this can be viewed as a p-adic uniformization of the component $\hat{\mathcal{C}}_{T}$ of the intersection.

We then have isomorphic fiber product diagrams of formal schemes over $W$ :

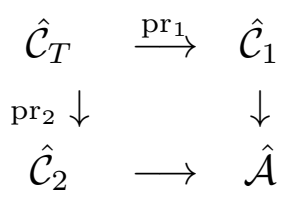

and

$$
\begin{array}{ccc}
Z^{\prime}(\mathbb{Q}) \backslash\left(Z \bullet(\mathbf{j}) \times I\left(\underline{x}, \omega_{1} \times \omega_{2}\right)\right) & \stackrel{\mathrm{pr}_{1}}{\longrightarrow} & H^{\prime}(\mathbb{Q})_{x_{1}} \backslash\left(Z \bullet\left(j_{1}\right) \times I\left(x_{1}, \omega_{1}\right)\right) \\
\downarrow & & \downarrow \\
\operatorname{pr}_{2} \downarrow & \longrightarrow & H^{\prime}(\mathbb{Q}) \backslash\left(\mathcal{M} \bullet \times H\left(\mathbb{A}_{f}^{p}\right) / K^{p}\right) .
\end{array}
$$

Observe that the projection maps $\operatorname{pr}_{i}$ factor as:

$$
\begin{array}{rlr}
Z^{\prime}(\mathbb{Q}) \backslash\left(Z \bullet(\mathbf{j}) \times I\left(\underline{x}, \omega_{1} \times \omega_{2}\right)\right) & \stackrel{\tilde{p r}_{i}}{\longrightarrow} & Z^{\prime}(\mathbb{Q}) \backslash\left(Z \bullet\left(j_{i}\right) \times I\left(x_{i}, \omega_{i}\right)\right) \\
\operatorname{pr}_{i} \searrow & \downarrow \\
& H^{\prime}(\mathbb{Q})_{x_{i}} \backslash\left(Z \bullet\left(j_{i}\right) \times I\left(x_{i}, \omega_{i}\right)\right) .
\end{array}
$$


We may assume that the element $\Pi$ chosen above lies in $B^{\prime}(\mathbb{Q})^{\times}=H^{\prime}(\mathbb{Q})$. Let $Z^{\prime}(\mathbb{Q})^{0}$ be the set of elements $z \in Z^{\prime}(\mathbb{Q})$ such that $\operatorname{ord}_{p}(\operatorname{det}(z))=0$, and note that $Z^{\prime}(\mathbb{Q})^{0}$ acts trivially on $\mathcal{M}^{\bullet}$. Then $Z^{\prime}(\mathbb{Q})=<\Pi^{2}>\times Z^{\prime}(\mathbb{Q})^{0}$, and, using the isomorphisms (8.20), we obtain an identification:

$Z^{\prime}(\mathbb{Q}) \backslash\left(Z^{\bullet}\left(j_{i}\right) \times I\left(x_{i}, \omega_{i}\right)\right) \simeq\left(Z\left(j_{i}\right) \times Z^{\prime}(\mathbb{Q})^{0} \backslash I\left(x_{i}, \omega_{i}\right)\right) \coprod\left(Z\left(j_{i}^{\vee}\right) \times Z^{\prime}(\mathbb{Q})^{0} \backslash I\left(x_{i}^{\vee}, \omega_{i}\right)\right)$.

There is an analogous decomposition

$$
\begin{aligned}
& Z^{\prime}(\mathbb{Q}) \backslash\left(Z^{\bullet}(\mathbf{j}) \times I\left(\underline{x}, \omega_{1} \times \omega_{2}\right)\right) \\
& \simeq\left(Z(\mathbf{j}) \times Z^{\prime}(\mathbb{Q})^{0} \backslash I\left(\underline{x}, \omega_{1} \times \omega_{2}\right)\right) \coprod\left(Z\left(\mathbf{j}^{\vee}\right) \times Z^{\prime}(\mathbb{Q})^{0} \backslash I\left(\underline{x}^{\vee}, \omega_{1} \times \omega_{2}\right)\right)
\end{aligned}
$$

for the right side of (8.32) and the decompositions (8.36) and (8.37) are compatible with the projections $\tilde{\mathrm{pr}}_{i}$.

Therefore the restriction of $\operatorname{pr}_{i}^{*} \mathcal{O}_{\hat{\mathcal{C}}_{i}}$ to the first (resp. second) component in the decomposition (8.37) is

$$
\tilde{p r}_{i}^{*} \mathcal{O}_{Z\left(j_{i}\right) \times Z^{\prime}(\mathbb{Q})^{0} \backslash I\left(x_{i}, \omega_{i}\right)}
$$

(resp.

$$
\left.\tilde{p r}_{i}^{*} \mathcal{O}_{Z\left(j_{i}^{\vee}\right) \times Z^{\prime}(\mathbb{Q})^{0} \backslash I\left(x_{i}^{\vee}, \omega_{i}\right)} \quad\right)
$$

Thus we have

$$
\begin{aligned}
\chi\left(\hat{\mathcal{C}}_{T}, \mathcal{O}_{\hat{\mathcal{C}}_{1}} \otimes^{\mathbb{L}} \mathcal{O}_{\hat{\mathcal{C}}_{2}}\right)= & \chi\left(Z(\mathbf{j}), \mathcal{O}_{Z\left(j_{1}\right)} \otimes^{\mathbb{L}} \mathcal{O}_{Z\left(j_{2}\right)}\right) \cdot\left|Z^{\prime}(\mathbb{Q})^{0} \backslash I\left(\underline{x}, \omega_{1} \times \omega_{2}\right)\right| \\
& +\chi\left(Z\left(\mathbf{j}^{\vee}\right), \mathcal{O}_{Z\left(j_{1}^{\vee}\right)} \otimes^{\mathbb{L}} \mathcal{O}_{Z\left(j_{2}^{\vee}\right)}\right) \cdot\left|Z^{\prime}(\mathbb{Q})^{0} \backslash I\left(\underline{x}^{\vee}, \omega_{1} \times \omega_{2}\right)\right| .
\end{aligned}
$$

Note that $I\left(x^{\vee}, \omega\right)=\Pi \cdot I(x, \omega)$ and that passing from $\underline{x}$ to $\underline{x}^{\vee}$ leaves the matrix of inner products of the components unchanged. Since the intersection number

$$
\left(Z\left(j_{1}\right), Z\left(j_{2}\right)\right)=\chi\left(Z(\mathbf{j}), \mathcal{O}_{Z\left(j_{1}\right)} \otimes^{\mathbb{L}} \mathcal{O}_{Z\left(j_{2}\right)}\right)
$$

depends only on the matrix $T$ of inner products, we have proved the following: 
Theorem 8.5. Assume that $\operatorname{det}(T) \neq 0$. Then

$$
\chi\left(\hat{\mathcal{C}}_{T}, \mathcal{O}_{\hat{\mathcal{C}}_{1}} \otimes^{\mathbb{L}} \mathcal{O}_{\hat{\mathcal{C}}_{2}}\right)=2 \cdot \chi\left(Z(\mathbf{j}), \mathcal{O}_{Z\left(j_{1}\right)} \otimes^{\mathbb{L}} \mathcal{O}_{Z\left(j_{2}\right)}\right) \cdot\left|Z^{\prime}(\mathbb{Q})^{0} \backslash I\left(\underline{x}, \omega_{1} \times \omega_{2}\right)\right| .
$$

This result is analogous to Theorem 14.11 of $[\mathbf{1 5}]$ and Theorem 7.2 of [16] in that it expresses the intersection number as a product of a multiplicity and a counting function. The analysis in our present case is much more elaborate, however, since the "multiplicity" is the intersection number $\chi\left(Z(\mathbf{j}), \mathcal{O}_{Z\left(j_{1}\right)} \otimes^{\mathbb{L}} \mathcal{O}_{Z\left(j_{2}\right)}\right)$, which is global on the Drinfeld space!

To obtain the final formula for the intersection number, we express the counting function as an orbital integral. Let $\varphi_{i}^{p} \in S\left(V\left(\mathbb{A}_{f}^{p}\right)\right)$ be the characteristic function of $\omega_{i}$, so that $\varphi_{1}^{p} \otimes \varphi_{2}^{p} \in S\left(V\left(\mathbb{A}_{f}^{p}\right)^{2}\right)$. Then, for $\underline{x} \in V\left(\mathbb{A}_{f}^{p}\right)_{T}^{2}$, the cardinality $\left|Z^{\prime}(\mathbb{Q})^{0} \backslash I\left(\underline{x}, \omega_{1} \times \omega_{2}\right)\right|$ is given by the orbital integral

$$
\begin{aligned}
\left|Z^{\prime}(\mathbb{Q})^{0} \backslash I\left(\underline{x}, \omega_{1} \times \omega_{2}\right)\right| & =\operatorname{vol}\left(K^{p}\right)^{-1} O_{T}\left(\varphi_{1}^{p} \otimes \varphi_{2}^{p}\right) \\
= & \operatorname{vol}\left(K^{p}\right)^{-1} \int_{Z\left(\mathbb{A}_{f}^{p}\right) \backslash H\left(\mathbb{A}_{f}^{p}\right)} \varphi_{1}^{p}\left(g^{-1} x_{1} g\right) \varphi_{2}^{p}\left(g^{-1} x_{2} g\right) d g .
\end{aligned}
$$

Combining this fact with the explicit formula for $e_{p}(T)=\left(Z\left(j_{1}\right), Z\left(j_{2}\right)\right)$ of Theorem 6.1 , which, we recall, depends only on the $G L_{2}\left(\mathbb{Z}_{p}\right)$-equivalence class of $T$, we obtain the following explicit expression for the intersection number.

Theorem 8.6. If $t_{1} t_{2}$ is not a square in $\mathbb{Q}^{\times}$, then the cycles $\mathcal{C}\left(t_{1}, \omega_{1}\right)$ and $\mathcal{C}\left(t_{2}, \omega_{2}\right)$ meet only in the special fiber of $\mathcal{A}_{K}$, and their intersection number is given by

$$
\left(\mathcal{C}\left(t_{1}, \omega_{1}\right), \mathcal{C}\left(t_{2}, \omega_{2}\right)\right)=2 \cdot \sum_{T} e_{p}(T) \cdot \operatorname{vol}\left(K^{p}\right)^{-1} O_{T}\left(\varphi_{1}^{p} \otimes \varphi_{2}^{p}\right)
$$

Here the sum runs over

$$
T=\left(\begin{array}{cc}
t_{1} & n \\
n & t_{2}
\end{array}\right) \in \operatorname{Sym}_{2}\left(\mathbb{Z}_{(p)}\right)
$$

$O_{T}\left(\varphi_{1}^{p} \otimes \varphi_{2}^{p}\right)$ is the orbital integral (8.41), and, if $T$ is $G L_{2}\left(\mathbb{Z}_{p}\right)$-equivalent to $\operatorname{diag}\left(\varepsilon_{1} p^{\alpha}, \varepsilon_{2} p^{\beta}\right)$, with $0 \leq \alpha \leq \beta$, then

$$
e_{p}(T)=\alpha+\beta+1- \begin{cases}p^{\alpha / 2}+2 \frac{p^{\alpha / 2}-1}{p-1} & \text { if } \alpha \text { is even and } \chi\left(\varepsilon_{1}\right)=-1, \\ (\beta-\alpha+1) p^{\alpha / 2}+2 \frac{p^{\alpha / 2}-1}{p-1} & \text { if } \alpha \text { is even and } \chi\left(\varepsilon_{1}\right)=1, \\ 2 \frac{p^{(\alpha+1) / 2}-1}{p-1} & \text { if } \alpha \text { is odd, }\end{cases}
$$


as in Theorem 6.1.

If $t_{1} t_{2}$ is a square, then the quantity $\left(\mathcal{C}\left(t_{1}, \omega_{1}\right), \mathcal{C}\left(t_{2}, \omega_{2}\right)\right)^{\mathrm{ns}}$ is defined by (8.26), and

$$
\left(\mathcal{C}\left(t_{1}, \omega_{1}\right), \mathcal{C}\left(t_{2}, \omega_{2}\right)\right)^{\mathrm{ns}}=2 \cdot \sum_{T, \operatorname{det}(T) \neq 0} e_{p}(T) \cdot \operatorname{vol}\left(K^{p}\right)^{-1} O_{T}\left(\varphi_{1}^{p} \otimes \varphi_{2}^{p}\right)
$$

\section{$\S$. Intersection numbers and Fourier coefficients.}

In this section, we combine the results of sections 7 and 8 to prove a relation between the intersection numbers of special cycles and the Fourier coefficients of the derivative of a certain Siegel Eisenstein series. This relation extends Theorem 14.11 of $[\mathbf{1 5}]$ to the primes of bad reduction $p \mid D(B)$ of the Shimura curve $\mathcal{A}_{K}$. We refer to section 7 of [15] for more details concerning the definition of the incoherent Siegel Eisenstein series and its derivative.

We continue to use the notation of section 8, with one exception. To make our results consistent with those of $[\mathbf{1 5}]$, we will change the sign of the quadratic form on $V$, i.e., we now take the quadratic form $Q(x)=\nu(x)$. Thus, a special cycle will be associated to data $(t, \omega)$ where $t \in \mathbb{Q}_{>0}^{\times}$with $\operatorname{ord}_{p}(t) \geq 0$ and where $\omega$ is a $K^{p}$-stable compact open subset of $V\left(\mathbb{A}_{f}^{p}\right)$. We also assume that $\omega$ is locally centrally symmetric.

For a pair of cycles $\mathcal{C}_{1}=\mathcal{C}\left(t_{1}, \omega_{1}\right)$ and $\mathcal{C}_{2}=\mathcal{C}\left(t_{2}, \omega_{2}\right)$, with $t_{1} t_{2}$ not a square in $\mathbb{Q}^{\times}$, let

$$
<\mathcal{C}_{1}, \mathcal{C}_{2}>_{p}:=\operatorname{vol}(K) \log (p)\left(\mathcal{C}_{1}, \mathcal{C}_{2}\right)
$$

be the $p$ part of their height pairing. Here $K=K_{p} K^{p}$ is the compact open subgroup of section 8 , and the intersection number $\left(\mathcal{C}_{1}, \mathcal{C}_{2}\right)$ is as in $(8.25)$. If $t_{1} t_{2}$ is a square, let

$$
<\mathcal{C}_{1}, \mathcal{C}_{2}>_{p}^{\mathrm{ns}}:=\operatorname{vol}(K) \log (p)\left(\mathcal{C}_{1}, \mathcal{C}_{2}\right)^{\mathrm{ns}}
$$

be the 'nonsingular part' of the height pairing, defined using (8.26). Of course, (9.2) simply reduces to (9.1) when $t_{1} t_{2}$ is not a square. Note that the intersection number $\left(\mathcal{C}_{1}, \mathcal{C}_{2}\right)$ is taken on the quotient $\mathcal{A}_{K}$ and hence depends on the choice of $K$. On the other hand, due to the factor $\operatorname{vol}(K)$, the quantity $\left\langle\mathcal{C}_{1}, \mathcal{C}_{2}\right\rangle_{p}$ is independent of $K$, but depends on the choice of Haar measure on $H\left(\mathbb{A}_{f}\right)$ used to calculate $\operatorname{vol}(K)$. Here we fix the Tamagawa measure $d h$ on $H(\mathbb{A})$ and a 
factorization of this measure as $d h=d_{\infty} h \cdot d_{f} h$ as in [15], p. 573. The Haar measure $d_{f} h$ on $H\left(\mathbb{A}_{f}\right)$ is used to compute $\operatorname{vol}(K)$. In addition, measures $d_{\ell} h$ are fixed for all $\ell$.

Next we introduce the relevant Eisenstein series. Let $\varphi_{1}^{p}$ and $\varphi_{2}^{p} \in S\left(V\left(\mathbb{A}_{f}^{p}\right)\right)^{K^{p}}$ be the characteristic functions of the sets $\omega_{1}$ and $\omega_{2}$. Let $\Phi_{f}^{p}(s)$ be the standard section of the induced representation $I_{2, \mathbb{A}_{f}^{p}}(s)$ with $\Phi_{f}^{p}(0)=\lambda_{f}^{p}\left(\varphi_{1}^{p} \otimes \varphi_{2}^{p}\right)$. Let $\tilde{\Phi}_{p}(s)$ be the nonstandard section of $I_{2, p}(s)$ defined in Corollary 7.4, and let $\Phi_{\infty}^{\frac{3}{2}}(s)$ be the standard section of weight $\frac{3}{2}$ as in (7.14) of [15]. Then

$$
\Phi(s)=\Phi_{\infty}^{\frac{3}{2}}(s) \otimes \tilde{\Phi}_{p}(s) \otimes \Phi_{f}^{p}(s)
$$

is an incoherent section of the global induced representation of the metaplectic group $G_{\mathbb{A}}^{\prime \prime}$ of genus 2 . Let $E\left(g^{\prime \prime}, s, \Phi\right)$ be the associated incoherent Eisenstein series.

Let $G_{\mathbb{A}}^{\prime}$ be the metaplectic cover of $S p_{1}(\mathbb{A})$ and recall that there is a homomorphism $\iota: G_{\mathbb{A}}^{\prime} \times G_{\mathbb{A}}^{\prime} \rightarrow G_{\mathbb{A}}^{\prime \prime}$. Restricting this to the real points, let $g^{\prime \prime}=\iota\left(g_{1}^{\prime}, g_{2}^{\prime}\right)$, where $g_{1}^{\prime}$ and $g_{2}^{\prime} \in G_{\mathbb{R}}^{\prime}$. For $g^{\prime} \in G_{\mathbb{R}}^{\prime}$, let $W_{t}^{\frac{3}{2}}\left(g^{\prime}\right)$ be the holomorphic Whittaker function of Proposition 7.3 of [15].

Theorem 9.1. For $g_{1}^{\prime}$ and $g_{2}^{\prime} \in G_{\mathbb{R}}^{\prime}$, and with the notation just introduced,

$$
2 \pi^{2} W_{t_{1}}^{\frac{3}{2}}\left(g_{1}^{\prime}\right) W_{t_{2}}^{\frac{3}{2}}\left(g_{2}^{\prime}\right)<\mathcal{C}_{1}, \mathcal{C}_{2}>_{p}^{\mathrm{ns}}=\sum_{T} E_{T}^{\prime}\left(\iota\left(g_{1}^{\prime}, g_{2}^{\prime}\right), 0, \Phi\right),
$$

where the sum is on positive definite $T \in \operatorname{Sym}_{2}\left(\mathbb{Z}_{(p)}\right)$ with

$$
T=\left(\begin{array}{cc}
t_{1} & * \\
* & t_{2}
\end{array}\right)
$$

and with $\mu_{p}(T)=1$. Here the invariant $\mu_{p}(T)$ is defined in Proposition 7.1.

Remark. The condition $\mu_{p}(T)=1$ implies that $T$ is not represented by the quadratic form $Q$ on $V\left(\mathbb{Z}_{p}\right)$.

Note that this result is consistent with Theorem 14.11, for $p \nmid D(B)$, and Theorem 12.6, for $p=\infty$, of [15]. Thus the result of section 15 of that paper can be extended correspondingly. 
Proof. For $T \in \operatorname{Sym}_{2}(\mathbb{Q})$, with $\operatorname{det}(T) \neq 0$, and for $\operatorname{Re}(s)$ sufficiently large, the $T$-th Fourier coefficient of the incoherent Eisenstein series $E\left(g^{\prime \prime}, s, \Phi\right)$ has a product formula

$$
E_{T}\left(g^{\prime \prime}, s, \Phi\right)=W_{T, \infty}\left(g^{\prime \prime}, s, \Phi_{\infty}^{\frac{3}{2}}\right) \cdot W_{T, p}\left(e, s, \tilde{\Phi}_{p}\right) \cdot \prod_{\ell \neq p} W_{T, \ell}\left(e, s, \Phi_{\ell}\right) .
$$

We will write

$$
W_{T}\left(s, \Phi_{f}^{p}\right)=\prod_{\ell \neq p} W_{T, \ell}\left(e, s, \Phi_{\ell}\right)
$$

and we recall that this function, initially defined for $\operatorname{Re}(s)$ sufficiently large, has an entire analytic continuation, [15], p. 562. We will only be interested in those $T$ for which $W_{T, p}\left(e, 0, \tilde{\Phi}_{p}\right)=0$, so that for the derivative

$$
E_{T}^{\prime}\left(g^{\prime \prime}, 0, \Phi\right)=W_{T, \infty}\left(g^{\prime \prime}, 0, \Phi_{\infty}^{\frac{3}{2}}\right) \cdot W_{T, p}^{\prime}\left(e, 0, \tilde{\Phi}_{p}\right) \cdot W_{T}\left(0, \Phi_{f}^{p}\right) .
$$

We would like to prove the identity

$$
\begin{aligned}
& E_{T}^{\prime}\left(\iota\left(g_{1}^{\prime}, g_{2}^{\prime}\right), 0, \Phi\right) \\
& =C \cdot W_{t_{1}}^{\frac{3}{2}}\left(g_{1}^{\prime}\right) W_{t_{2}}^{\frac{3}{2}}\left(g_{2}^{\prime}\right) \operatorname{vol}(K) \log (p)\left(\mathcal{C}_{1}, \mathcal{C}_{2}\right)_{-T} \\
& =C \cdot W_{t_{1}}^{\frac{3}{2}}\left(g_{1}^{\prime}\right) W_{t_{2}}^{\frac{3}{2}}\left(g_{2}^{\prime}\right) \operatorname{vol}(K) \log (p) 2 e_{p}(-T) \cdot \operatorname{vol}\left(K^{p}\right)^{-1} O_{T}\left(\varphi_{1}^{p} \otimes \varphi_{2}^{p}\right),
\end{aligned}
$$

with constant $C=2 \pi^{2}$. By (7.35) of [15], we have

$$
W_{T, \infty}\left(g^{\prime \prime}, 0, \Phi_{\infty}^{\frac{3}{2}}\right)=W_{t_{1}}^{\frac{3}{2}}\left(g_{1}^{\prime}\right) W_{t_{2}}^{\frac{3}{2}}\left(g_{2}^{\prime}\right)
$$

while, by Corollary 7.4 above,

$$
W_{T, p}^{\prime}\left(e, 0, \tilde{\Phi}_{p}\right)=\frac{p+1}{p^{2}} \log (p) e_{p}(-T) .
$$

Thus, substituting these in (9.7), we find that the desired identity is:

$$
\frac{p+1}{p^{2}} \cdot W_{T}\left(0, \Phi_{f}^{p}\right)=2 C \cdot \operatorname{vol}\left(K_{p}\right) \cdot O_{T}\left(\varphi_{f}^{p}\right),
$$

where $\varphi_{f}^{p}=\varphi_{1}^{p} \otimes \varphi_{2}^{p}$. 
An easy calculation shows that $\operatorname{vol}\left(K_{p}\right)=(p+1) / p^{2}$, so that it remains to show that

$$
W_{T}\left(0, \Phi_{f}^{p}\right)=2 C \cdot O_{T}\left(\varphi_{f}^{p}\right)
$$

This last identity can be derived from the Siegel-Weil formula as follows.

Let $B^{\prime}$ be the definite quaternion algebra defined in section 8 above and let $V^{\prime}$ be the space of trace 0 elements in $B^{\prime}$ with quadratic form $Q(x)=\nu(x)$ given by the restriction of the reduced norm. The identification (8.5) gives an identification

$$
V^{\prime}\left(\mathbb{A}_{f}^{p}\right)=V\left(\mathbb{A}_{f}^{p}\right)
$$

Let $\varphi^{\prime}=\otimes_{v} \varphi_{v}^{\prime} \in S\left(V^{\prime}(\mathbb{A})^{2}\right)$ be the factorizable, locally even, global Schwartz function defined by

$$
\varphi_{v}^{\prime}(x)= \begin{cases}e^{-\pi \operatorname{tr}(Q(x))} & \text { if } v=\infty, \\ \varphi_{1, \ell} \otimes \varphi_{2, \ell}(x) & \text { if } v=\ell \neq p, \\ \varphi_{p}^{\prime}(x) & \text { if } v=p,\end{cases}
$$

for some (for the moment arbitrary) $\varphi_{p}^{\prime} \in S\left(V^{\prime}\left(\mathbb{Q}_{p}\right)^{2}\right)$. If $T \in \operatorname{Sym}_{2}(\mathbb{Q})$ with $\operatorname{det}(T) \neq 0$ is represented by $V^{\prime}$, then, by (7.28) of [15], the $T$-th Fourier coefficient of the theta integral of $\varphi^{\prime}$ is given by

$$
\begin{aligned}
I_{T}\left(g^{\prime \prime}, \varphi^{\prime}\right) & =\frac{1}{2} \int_{Z^{\prime}(\mathbb{A}) H^{\prime}(\mathbb{Q}) \backslash H^{\prime}(\mathbb{A})} \sum_{\substack{x \in V^{\prime}(\mathbb{Q})^{2} \\
Q(x)=T}} \omega_{\psi}\left(g^{\prime \prime}\right) \varphi^{\prime}\left(h^{-1} x\right) d h \\
& =\frac{1}{2} \int_{Z^{\prime}(\mathbb{A}) \backslash H^{\prime}(\mathbb{A})} \omega_{\psi}\left(g^{\prime \prime}\right) \varphi^{\prime}\left(h^{-1} x_{0}\right) d h \\
& =\frac{1}{2} \cdot O_{T}\left(\omega_{\psi}\left(g^{\prime \prime}\right) \varphi_{\infty}^{\prime}\right) \cdot O_{T}\left(\varphi_{p}^{\prime}\right) \cdot O_{T}\left(\varphi_{f}^{p}\right) .
\end{aligned}
$$

Here $x_{0} \in V(\mathbb{Q})^{2}$ is an arbitrary base point, and the orbital integrals are formed as in (8.41). We take $\varphi_{p}^{\prime}$ even so that $\varphi^{\prime}$ is locally even.

Note that the individual factors here depend on the choice of the Haar measures, which are fixed as above. By the Siegel-Weil formula for the coherent Eisenstein series associated to $\lambda^{\prime}\left(\varphi^{\prime}\right)$,

$$
E_{T}\left(g^{\prime \prime}, 0, \Phi^{\prime}\right)=2 \cdot I_{T}\left(g^{\prime \prime}, \varphi^{\prime}\right)
$$


i.e.,

$W_{T, \infty}\left(g^{\prime \prime}, 0, \Phi_{\infty}^{\frac{3}{2}}\right) \cdot W_{T, p}\left(e, 0, \Phi_{p}^{\prime}\right) \cdot W_{T}\left(0, \Phi_{f}^{p}\right)=O_{T}\left(\omega_{\psi}\left(g^{\prime \prime}\right) \varphi_{\infty}^{\prime}\right) \cdot O_{T}\left(\varphi_{p}^{\prime}\right) \cdot O_{T}\left(\varphi_{f}^{p}\right)$.

By $(7.33)-(7.35)$ of $[\mathbf{1 5}]$ :

$$
O_{T}\left(\omega_{\psi}\left(g^{\prime \prime}\right) \varphi_{\infty}^{\prime}\right)=(2 \pi)^{2} W_{t_{1}}\left(g_{1}^{\prime}\right) W_{t_{2}}\left(g_{2}^{\prime}\right)
$$

so that (9.16) becomes

$$
W_{T, p}\left(e, 0, \Phi_{p}^{\prime}\right) \cdot W_{T}\left(0, \Phi_{f}^{p}\right)=(2 \pi)^{2} \cdot O_{T}\left(\varphi_{p}^{\prime}\right) \cdot O_{T}\left(\varphi_{f}^{p}\right)
$$

We now choose $\varphi_{p}^{\prime}$ so that $W_{T, p}\left(e, 0, \Phi_{p}^{\prime}\right) \neq 0$, and we compute the ratio.

\section{Lemma 9.2.}

$$
\frac{O_{T}\left(\varphi_{p}^{\prime}\right)}{W_{T, p}\left(e, 0, \Phi_{p}^{\prime}\right)}=1
$$

Proof. We only sketch the argument which consists of two steps. In the first step one proves that the ratio on the left hand side of Lemma 9.2 is independent of $T \in \operatorname{Sym}_{2}\left(\mathbb{Q}_{p}\right)$ (with $\operatorname{det}(T) \neq 0$ ) and of $\varphi_{p}^{\prime} \in S\left(V^{\prime}\left(\mathbb{Q}_{p}\right)^{2}\right)$. In the second step one calculates the ratio by making a special choice of $T$ and $\varphi_{p}^{\prime}$. Namely, if $T_{0}=-1_{2}$ and $\varphi_{0}=\operatorname{char} V^{\prime}\left(\mathbb{Z}_{p}\right)^{2}$, then

$$
O_{T_{0}}\left(\varphi_{0}\right)=\operatorname{vol}\left(K_{p}^{\prime}\right)
$$

With the measures as described on p. 573 of [15] the naive volume of $K_{p}^{\prime}$, i.e., without the convergence factor $\lambda_{p}=\left(1-p^{-1}\right)$ is

$$
p^{-4}\left(p^{2}-1\right)\left(p^{2}-p\right)
$$

so that, dividing by the convergence factor the right hand side of $(9.19)$ is $1-p^{2}$.

On the other hand, by Kitaoka's formula (cf. Proposition 8.3 of [15] and noting that $\left.\gamma\left(V^{\prime}\left(\mathbb{Q}_{p}\right)\right)=1\right)$

$$
W_{T_{0}}\left(e, 0, \Phi_{0}\right)=\alpha_{p}\left(S, T_{0}\right)=1-p^{-2}
$$

In particular,

$$
W_{T}\left(0, \Phi_{f}^{p}\right)=(2 \pi)^{2} \cdot O_{T}\left(\varphi_{f}^{p}\right)
$$

and the proof of Theorem 9.1 is complete. 


\section{References.}

[1] M. F. Atiyah and I. G. MacDonald, Introduction to Commutative Algebra, Addison-Wesley, Reading, MA, 1969.

[2] J.-F. Boutot and H. Carayol, Uniformisation p-adique des courbes de Shimura: les théorèmes de Cerednik et de Drinfeld, in: Courbes modulaires et courbes de Shimura, Astérisque 196-197, 1991, pp. 45-158.

[3] P. Deligne, Intersections sur les surfaces régulières, exposé $X$, in $\mathrm{P}$. Deligne and N. Katz, SGA 7, II: Groupes de monodromie en géométrie algébrique, SLN 340, Springer 1973, 1-38.

[4] P. Deligne, La classe de cohomologie associée à un cycle par A. Grothendieck, in P. Deligne: SGA 4 $\frac{1}{2}$, SLN 569, Springer 1973, 129-153.

[5] V. G. Drinfeld, Coverings of p-adic symmetric regions, Funct. Anal. Appl. 10 (1977), 29-40.

[6] A. Genestier, Espaces Symétriques de Drinfeld, Astérisque 234, 1996.

[7] A. Genestier, Letter to M. Rapoport, August 12, 1996.

[8] B. H. Gross, On canonical and quasi-canonical liftings, Inventiones math. 84 (1986), 321-326.

[9] B. H. Gross and K. Keating, On the intersection of modular correspondences, Inventiones math. 112 (1993), 225-245.

[10] H. Katsurada, An explicit formula for the Fourier coefficients of Siegel-Eisenstein series, preprint (1997).

[11] Y. Kitaoka, A note on local densities of quadratic forms, Nagoya Math. J. 92 (1983), 145-152.

[12] Y. Kitaoka, Fourier coefficients of Eisenstein series of degree 3, Proc. Japan Acad. 60 (1984), 259-261.

[13] R. Kottwitz, Points on some Shimura varieties over finite fields, JAMS 5 (1992), 373-444. 
[14] R. Kottwitz, Calculation of some orbital integrals, R.P. Langlands and D. Ramakrishnan (ed.), The zeta functions of Picard modular surfaces, Publ. CRM. Montreal (1992), 349-362.

[15] S. Kudla, Central derivatives of Eisenstein series and height pairings, Annals of Math. 146 (1997), 545-646.

[16] S. Kudla and M. Rapoport, Cycles on Siegel 3-folds and derivatives of Eisenstein series, preprint (1997).

[17] S. Kudla and M. Rapoport, Arithmetic Hirzebruch-Zagier cycles, in preparation.

[18] B. Myers, Local representation densities of non-unimodular quadratic forms, thesis, University of Maryland (1994).

[19] M. Rapoport and T. Zink, Periods of p-divisible groups, Annals of Math. Studies 141, Princeton U. Press, Princeton, NJ, 1996.

[20] Tonghai Yang, An explicit formula for local densities of quadratic forms, to appear in J. Number Theory.

[21] T. Zink, Über die schlechte Reduktion einiger Shimuramannigfaltigkeiten, Compositio Math. 45 (1981), 15-107.

Stephen S. Kudla

Department of Mathematics

University of Maryland

College Park, MD 20742
Michael Rapoport Mathematisches Institut der Universität zu Köln Weyertal 86-90 D - 50931 Köln Germany

USA 